\title{
Spitzer infrared spectrograph point source classification in the Small Magellanic Cloud
}

Paul M. E. Ruffle, ${ }^{1,2 \star}$ F. Kemper ${ }^{2} \dagger$ O. C. Jones, ${ }^{1,3}$ G. C. Sloan, ${ }^{4}$ K. E. Kraemer, ${ }^{5}$ Paul M. Woods, ${ }^{6}$ M. L. Boyer, ${ }^{7}$ S. Srinivasan, ${ }^{2}$ V. Antoniou, ${ }^{8}$ E. Lagadec, ${ }^{9}$ M. Matsuura, ${ }^{10,11}$ I. McDonald, ${ }^{1}$ J. M. Oliveira, ${ }^{12}$ B. A. Sargent, ${ }^{13}$ M. Sewiło, ${ }^{14,15}$ R. Szczerba, ${ }^{16}$ J. Th. van Loon, ${ }^{12}$ K. Volk ${ }^{3}$ and A. A. Zijlstra ${ }^{1}$

${ }^{1}$ Jodrell Bank Centre for Astrophysics, The University of Manchester, Alan Turing Building, Oxford Road, Manchester M13 9PL, UK

${ }^{2}$ Academia Sinica, Institute of Astronomy and Astrophysics, Taipei 10617, Taiwan

${ }^{3}$ Space Telescope Science Institute, 3700 San Martin Drive, Baltimore, MD 21218, USA

${ }^{4}$ Department of Astronomy, Cornell University, Ithaca, NY 14853, USA

${ }^{5}$ Institute for Scientific Research, Boston College, 140 Commonwealth Avenue, Chestnuthill, MA 02467, USA

${ }^{6}$ Astrophysics Research Centre, School of Mathematics \& Physics, Queen's University Belfast, University Road, Belfast BT7 1NN, UK

${ }^{7}$ Observational Cosmology Lab, Code 665, NASA Goddard Space Flight Center, Greenbelt, MD 20771, USA

${ }^{8}$ Harvard-Smithsonian Center for Astrophysics, 60 Garden Street, Cambridge, MA 02138, USA

${ }^{9}$ Laboratoire Lagrange, Université de Nice - Sophia Antipolis, Observatoire de la Côte d'Azur, CNRS, F-06304 Nice, France

${ }^{10}$ School of Physics and Astronomy, Cardiff University, The Parade, Cardiff CF24 3AA, UK

${ }^{11}$ Department of Physics and Astronomy, University College London, Gower Street, London WC1E 6BT, UK

${ }^{12}$ School of Physical and Geographical Sciences, Lennard-Jones Laboratories, Keele University, Staffordshire ST5 5BG, UK

${ }^{13}$ Laboratory for Multiwavelength Astrophysics, Rochester Institute of Technology, 54 Lomb Memorial Drive, Rochester, NY 14623, USA

${ }^{14}$ Space Science Institute, 4750 Walnut Street, Suite 205, Boulder, CO 80301, USA

${ }^{15}$ Department of Physics and Astronomy, Johns Hopkins University, 366 Bloomberg Center, 3400 N. Charles Street, Baltimore, MD 21218 , USA

${ }^{16}$ N. Copernicus Astronomical Center, Rabianska 8, PL-87-100 Torun, Poland

\begin{abstract}
The Magellanic Clouds are uniquely placed to study the stellar contribution to dust emission. Individual stars can be resolved in these systems even in the mid-infrared, and they are close enough to allow detection of infrared excess caused by dust. We have searched the Spitzer Space Telescope data archive for all Infrared Spectrograph (IRS) staring-mode observations of the Small Magellanic Cloud (SMC) and found that 209 Infrared Array Camera (IRAC) point sources within the footprint of the Surveying the Agents of Galaxy Evolution in the Small Magellanic Cloud (SAGE-SMC) Spitzer Legacy programme were targeted, within a total of 311 staring-mode observations. We classify these point sources using a decision tree method of object classification, based on infrared spectral features, continuum and spectral energy distribution shape, bolometric luminosity, cluster membership and variability information. We find 58 asymptotic giant branch (AGB) stars, 51 young stellar objects, 4 post-AGB objects, 22 red supergiants, 27 stars (of which 23 are dusty OB stars), 24 planetary nebulae (PNe), 10 Wolf-Rayet stars, $3 \mathrm{H}$ II regions, 3 R Coronae Borealis stars, 1 Blue Supergiant and 6 other objects, including 2 foreground AGB stars. We use these classifications to evaluate the success of photometric classification methods reported in the literature.
\end{abstract}

Key words: techniques: spectroscopic-surveys - dust, extinction - $\mathrm{H}$ II regions - Magellanic Clouds - infrared: stars.

${ }^{\star}$ Paul M. E. Ruffle passed away on 21 November 2013. His co-authors have finished the manuscript on his behalf, and would like to dedicate it to his memory. Paul was a very enthusiastic scientist, and a wonderful friend with a great sense of humour. We miss him tremendously.

†E-mail: ciska@asiaa.sinica.edu.tw

\section{INTRODUCTION}

The Mega-Surveying the Agents of Galaxy Evolution (MegaSAGE) project has obtained infrared photometric and spectroscopic inventories of the Magellanic Clouds with the Spitzer Space Telescope (hereafter Spitzer), using Spitzer and Herschel Legacy Programmes. The initial SAGE survey (Meixner et al. 2006) detected 
Table 1. IRS staring-mode targets in the SMC. The first 10 lines are presented to demonstrate the format of this table; the full table is available online.

\begin{tabular}{cccccll}
\hline SMC IRS & AOR & PID & PI & \multicolumn{2}{c}{ Spectrum position } & CASSIS file name \\
& & & & RA $(\mathrm{h} \mathrm{m} \mathrm{s})$ & Dec. $\left({ }^{\prime \prime \prime}{ }^{\prime \prime}\right)$ & \\
\hline 1 & 3824640 & 18 & Houck & 004640.32 & -730610.80 & NULL \\
2 & 3824896 & 18 & Houck & 010916.80 & -731203.60 & NULL \\
3 & 4384000 & 63 & Houck & 012407.68 & -730903.60 & NULL \\
4 & 4384000 & 63 & Houck & 012407.68 & -730903.60 & NULL \\
5 & 4384000 & 63 & Houck & 012407.68 & -730903.60 & NULL \\
6 & 4384768 & 63 & Houck & 005909.84 & -721051.60 & NULL \\
7 & 4385024 & 63 & Houck & 005852.25 & -720925.92 & cassis_tbl_spcf_4385024_1.tbl \\
8 & 4385024 & 63 & Houck & 005858.22 & -720950.76 & cassis_tbl_spcf_4385024_2.tbl \\
9 & 4385024 & 63 & Houck & 005858.80 & -721025.32 & cassis_tbl_spcf_4385024_3.tbl \\
10 & 4385024 & 63 & Houck & 005906.62 & -721025.68 & cassis_tbl_spcf_4385024_4.tbl \\
\hline
\end{tabular}

and catalogued $\sim 6.9$ million point sources in the Large Magellanic Cloud (LMC), ${ }^{1}$ while the SAGE-SMC survey (Gordon et al. 2011) detected and catalogued $\sim 2.2$ million point sources in the Small Magellanic Cloud (SMC). ${ }^{2}$ Both surveys used all bands of the Infrared Array Camera (IRAC; 3.6, 4.5, 5.8, $8.0 \mu \mathrm{m}$; Fazio et al. 2004) and the Multi-Band Imaging Photometer for Spitzer (MIPS; 24, 70, $160 \mu \mathrm{m}$; Rieke et al. 2004) instruments on board Spitzer (Werner et al. 2004). The resolution of the IRAC observations is $\sim 2$ arcsec, while for the MIPS bands, three different resolutions apply: 6,18 , and 40 arcsec for the 24,70 , and $160 \mu \mathrm{m}$ bands, respectively (Gordon et al. 2011). To follow up on these programmes, the SAGE-Spec project (Kemper et al. 2010) obtained 196 staring-mode pointings using Spitzer's Infrared Spectrograph (IRS; Houck et al. 2004, 5.2-38 $\mu \mathrm{m}$ ) of positions selected from the SAGE catalogue. SAGE-Spec will relate SAGE photometry to the spectral characteristics of different types of objects in both Magellanic Clouds, and ultimately, allow us to classify photometric point sources in both the LMC and SMC. This characterization of the point sources observed in the SAGE-Spec survey, and the IRS data archive, builds an inventory of dusty sources and their interrelation in each of the Magellanic Clouds. In the first step towards this goal, Woods et al. (2011) classified the initial 196 LMC point sources, using a decision tree method of object classification, based on infrared (IR) spectral features, continuum and spectral energy distribution (SED) shape, bolometric luminosity, cluster membership, and variability information. The initial classification of LMC objects is being extended to $\sim 1000$ point sources, covering all archival IRS observations within the SAGE footprint (Woods et al., in preparation).

To extend the LMC classifications to the SMC, we have searched the Spitzer data archive for IRS staring-mode observations and found 311 spectra, yielding 209 unique and genuine point sources with IRS data within the footprint of the SAGE-SMC Spitzer Legacy programme. The data used in the classification process are described in Section 2. In Section 3, we discuss the classification method, and in Section 4 we classify the 209 SMC point sources using the decision tree method. Finally, in Section 5 we compare spectral versus colour classifications by means of colour-magnitude diagrams (CMDs). We use our spectroscopic classifications to test photometric classification methods, e.g. those by Boyer et al. (2011), Sewiło et al. (2013), and Matsuura, Woods \& Owen (2013). The classifi-

\footnotetext{
${ }^{1}$ http://irsa.ipac.caltech.edu/data/SPITZER/docs/spitzermission/ observingprograms/legacy/sage

2 http://irsa.ipac.caltech.edu/data/SPITZER/docs/spitzermission/ observingprograms/legacy/sagesmc
}

cation of each of these 209 sources is part of the data delivery of the SAGE-Spec Legacy project to the Spitzer Science Center and the community. ${ }^{3}$ These classifications will also be used to benchmark a colour-classification scheme that will be applied to all point sources in the SAGE and SAGE-SMC surveys (Marengo et al., in preparation).

\section{DATA PREPARATION}

\subsection{Spitzer IRS staring-mode observations}

The IRS on board Spitzer covers the wavelength range 5-38 $\mu \mathrm{m}$. For the low-resolution mode, the spectrum splits in two bands, short-

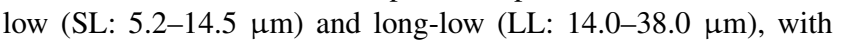
almost perpendicular slits. Each segment splits into a range covered at second order (SL2, LL2), and one at first order (SL1, LL1). The resolution varies between 60 and 130. The high-resolution mode covers the wavelength range from 10 to $19.6 \mu \mathrm{m}$ (short-high; $\mathrm{SH}$ ) and from 18.7 to $37.2 \mu \mathrm{m}$ (long-high; $\mathrm{LH}$ ) with a spectral resolution of $R \sim 600$.

We identified 311 Spitzer IRS low- and high-resolution staringmode observations within the footprint of the SAGE-SMC survey (Gordon et al. 2011), not necessarily associated with a point source. We numbered these SMC IRS 1-311, by ordering them by observing programme (Project ID; PID) first, and then the Astronomical Observation Request (AOR) number (Table 1). Where available (for SL and LL observations only), the reduced spectra were downloaded from the Cornell Atlas of Spitzer IRS Sources ${ }^{4}$ (CASSIS; Lebouteiller et al. 2011), in a full resolution grid, using the optimal extraction method. At the moment, the CASSIS data base only contains SL and LL data, but it turns out that there are no point sources in the SMC targeted with only SH and LH, so we will use the SL and LL data only. The data were downloaded in the Infrared Processing and Analysis Center (IPAC) table format; ${ }^{5}$ the file names are also given in Table 1. As an example, the upper-left panel of Fig. 1 shows the spectrum for point source SMC IRS 110, with the SL2 and LL2 data from CASSIS in red and the SL1 and LL1 data in blue.

The CASSIS-reduced IRS data header provides the original PI's requested (REQ) position and the field-of-view (FOV) position,

\footnotetext{
3 http://irsa.ipac.caltech.edu/data/SPITZER/docs/spitzermission/ observingprograms/legacy/sagespec

${ }^{4}$ http://cassis.astro.cornell.edu

${ }^{5} \mathrm{http}$ ://irsa.ipac.caltech.edu/applications/DDGEN/Doc/ipac_tbl.html
} 

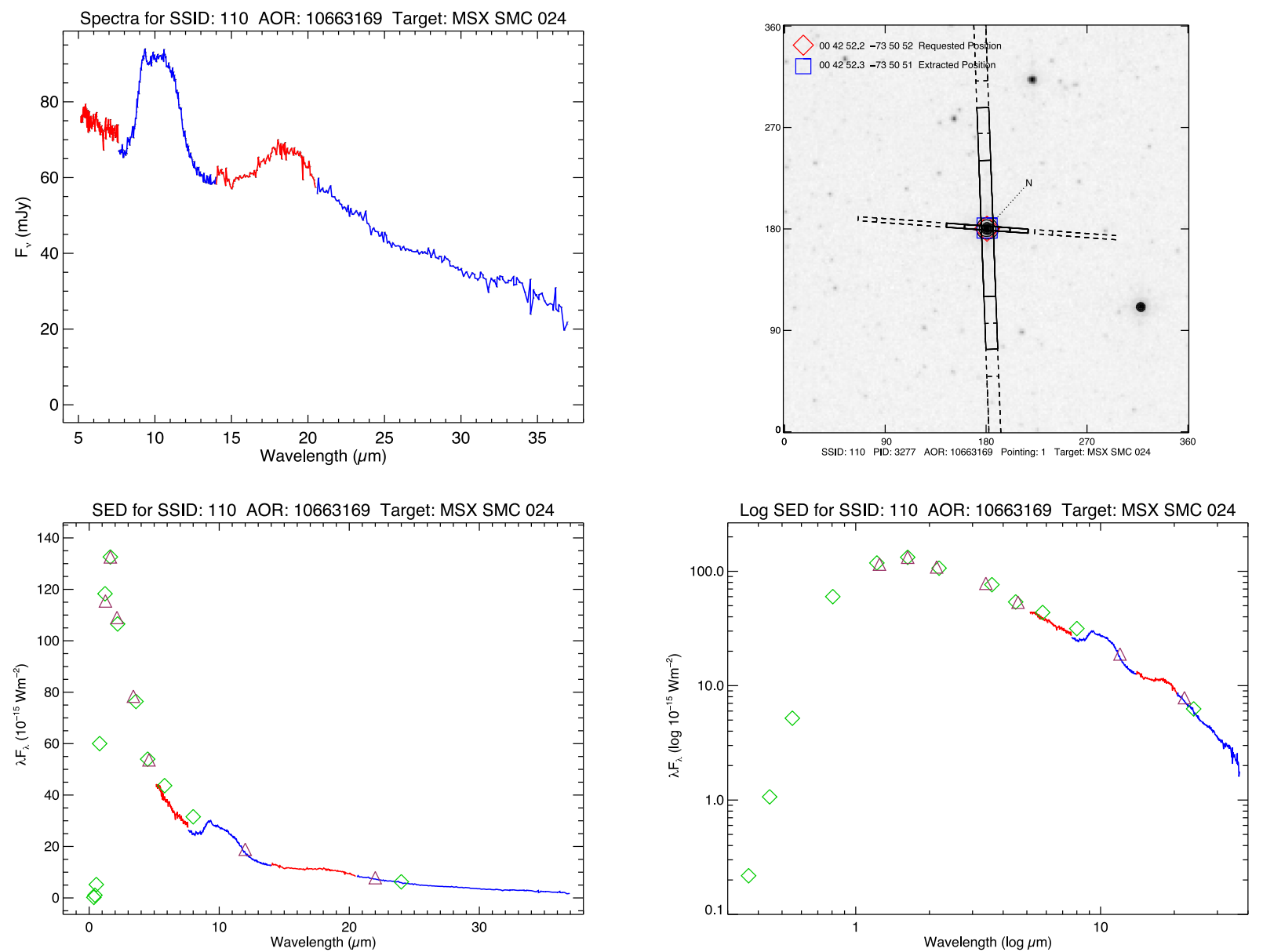

Figure 1. From upper-left to lower-right: example spectrum, slit image, SED and log SED plots for point source SMC IRS 110. The spectrum plots were generated using CASSIS-reduced IRS data from the following low-resolution modules: SL second order 5.2-7.6 $\mu \mathrm{m}$ (red); SL first order 7.6-14.0 $\mu \mathrm{m}$ (blue); LL second order 14.0-20.5 $\mu \mathrm{m}$ (red); LL first order 20.5-37.0 $\mu \mathrm{m}$ (blue). Slit images were created by overplotting IRS SL and LL slit positions on a 360 arcsec $\times 360$ arcsec image extracted from the SAGE-SMC $8 \mu \mathrm{m}$ image; the REQ and EXT or FOV positions were also overplotted. SED and log SED plots (lower-left and lower-right panels, respectively) were generated by combining the above IRS data with the following photometric data points: SAGE-SMC catalogue (green diamonds): $U, B, V, I$ (MCPS), $J, H, K_{S}$ (IRSF); 3.6, 4.5, 5.8, $8.0 \mu \mathrm{m}$ (IRAC), $24 \mu \mathrm{m}$ (MIPS); WISE catalogue (magenta triangles): $J, H, K_{S}$ (2MASS), 3.4, 4.6, 12, $22 \mu \mathrm{m}$ (WISE).

i.e. where the telescope actually pointed (usually, but not always coincident within 1 arcsec of the REQ position). In many cases, however, neither of these two positions is the same as the position at which the spectrum is actually extracted from the slit (EXT), using the optimal extraction method. In order to check the spectrum position for each source, slit images were generated by overplotting the IRS SL and LL slit positions (recorded in each Spitzer AOR's BCD FITS header) on a 360 arcsec $\times 360$ arcsec image extracted from the SAGE-SMC $8 \mu \mathrm{m}$ image. The REQ and EXT positions were also overplotted on the image. In cases where the spectrum was extracted at the FOV position (and thus an EXT position is lacking), the FOV position was overplotted instead (see upper-right panel of Fig. 1 for an example for SMC IRS 110). The slit images are useful in determining the origin of the emission seen in the IRS spectra. The coordinates in Table 1 represent, if available, the EXT position. The next preference is the FOV position, and if neither of these is available, the REQ position is given.

\subsection{Photometric matching}

In order to find matching photometry for the IRS spectra, we searched the SAGE-SMC Single Frame + Mosaic Photometry
(SMP) Archive v1.5 (Gordon et al. 2011) available on Gator, ${ }^{6}$ using, in order of preference, the EXT, FOV or REQ spectrum positions. We searched for IRAC point source matches within 3 arcsec of the spectrum positions, which corresponds with the pointing accuracy of the IRS mode on Spitzer. In cases where the SAGESMC point source catalogue (PSC) did not provide a match, we also searched the Spitzer Survey of the Small Magellanic Cloud ( $\mathrm{S}^{3} \mathrm{MC}$ ) catalogue ${ }^{7}$ (Bolatto et al. 2007) for IRAC matches within 3 arcsec. We found three sources in $\mathrm{S}^{3} \mathrm{MC}$ without a SAGE-SMC catalogue counterpart. Although the $S^{3} \mathrm{MC}$ data are included in the SAGE-SMC result, both teams used different point source extraction pipelines and the source catalogues therefore do not provide a one-to-one match.

\footnotetext{
${ }^{6}$ Gator is the general catalogue query engine provided by the NASA/IPAC Infrared Science Archive, which is operated by the Jet Propulsion Laboratory, California Institute of Technology, under contract with the National Aeronautics and Space Administration.

${ }^{7}$ At the time of writing only the $\mathrm{S}^{3} \mathrm{MC}$ YSO Catalog is available in the public domain (Bolatto, priv. communication).
} 
Table 2. Numbering, names and description of the columns present in the classification table which is available online only.

\begin{tabular}{|c|c|c|}
\hline Column & Name & Description \\
\hline 1 & smc_irs & SMC IRS identification number of the target \\
\hline 2 & name & Name of point source targeted \\
\hline 3 & sage_spec_class & Source classification determined in this paper \\
\hline $4-5$ & ra_spec, dec_spec & Position of the extracted spectrum \\
\hline 6 & aor & Spitzer AOR number \\
\hline 7 & pid & Spitzer observing programme identification number \\
\hline 8 & pi & Last name of the PI of the Spitzer PID \\
\hline 9 & cassis_file_name & Name of the file containing the CASSIS-reduced Spitzer-IRS spectrum \\
\hline 10 & irac_des & $\begin{array}{l}\text { SAGE-SMC or } \mathrm{S}^{3} \mathrm{MC} \text { IRAC point source designation matching the } \\
\text { extracted spectrum }\end{array}$ \\
\hline $11-12$ & ra_ph, dec_ph & RA and Dec. in degrees of the IRAC point source \\
\hline 13 & dpos_ph & $\begin{array}{l}\text { Distance in arcsec between the IRAC point source and the position of the } \\
\text { extracted spectrum }\end{array}$ \\
\hline $14-17$ & irac1, irac2, irac3, irac4 & IRAC magnitudes in bands $1-4$ \\
\hline $18-20$ & tycho_des, b_tycho, v_tycho & TYCHO counterpart and its $B$ and $V$ magnitudes \\
\hline $21-25$ & $\begin{array}{l}\text { m2002_des, u_m2002, b_m2002, v_m2002, } \\
\text { r_m2002 }\end{array}$ & Massey (2002) counterpart and its $U, B, V, R$ magnitudes \\
\hline $26-29$ & u_mcps, b_mcps, v_mcps, i_mcps & Matching MCPS $U, B, V, I$ magnitudes \\
\hline $30-33$ & denis_des, i_denis, j_denis, $\mathrm{k} \_$denis & DENIS counterpart and its $I, J, K_{S}$ magnitudes \\
\hline $34-37$ & irsf_des, j_irsf, h_irsf, k_irsf & IRSF counterpart and its $J, H, K_{S}$ magnitudes \\
\hline $38-41$ & tmass_des, j_tmass, h_tmass, $\mathrm{k} \_t$ mass & 2MASS $6 \times$ counterpart and its $J, H, K_{S}$ magnitudes \\
\hline $42-46$ & wise_des, wise 1, wise2, wise 3, wise 4 & WISE counterpart and its magnitudes in the four WISE bands \\
\hline $47-52$ & $\begin{array}{l}\text { akari_n3, akari_n4, akari_s7, akari_s11, akari_115, } \\
\text { akari_124 }\end{array}$ & $\begin{array}{l}\text { Matching } A K A R I \text { magnitudes in bands N3, N4, S7, S11, L15 and L24 from } \\
\text { Ita et al. (2010) }\end{array}$ \\
\hline $53-54$ & mips24_des, mips24 & SAGE-SMC MIPS-[24] designation and magnitude \\
\hline $55-56$ & mips70_des, mips70 & SAGE-SMC MIPS-[70] designation and magnitude \\
\hline $57-58$ & mips160_des, mips160 & SAGE-SMC MIPS-[160] designation and magnitude \\
\hline 59 & mbol_phot & $\begin{array}{l}M_{\text {bol }} \text { calculated by interpolation of } J H K_{S}, \text { IRAC and MIPS-[24] } \\
\text { photometry, with a Wien and Rayleigh-Jeans tail }\end{array}$ \\
\hline 60 & mbol_phwi & As \#59, but with WISE photometry added \\
\hline 61 & mbol_phsp & As \#59, but with the IRS spectrum added \\
\hline 62 & mbol_pwsp & As \#59, but with the IRS spectrum and WISE photometry added \\
\hline $63-65$ & mbol_mcd, lum_mcd, teff_mcd & $\begin{array}{l}M_{\text {bol }} \text { calculated using the SED fitting code from McDonald et al. (2009), } \\
\text { McDonald, Zijlstra \& Boyer (2012), only good fits are included }\end{array}$ \\
\hline $66-67$ & id_groen, per_groen & $\begin{array}{l}\text { Source ID and variability period in days from } \\
\text { Groenewegen et al. (2009) }\end{array}$ \\
\hline $68-72$ & $\begin{array}{l}\text { ogle3id, ogle3mean_i, ogle3mean_v, ogle3amp_i, } \\
\text { ogle3period }\end{array}$ & Source ID and variability information from the OGLE survey \\
\hline 73 & boyer_class & Colour classification from Boyer et al. (2011) \\
\hline 74 & matsuura_class & Colour classification from Matsuura et al. (2013) \\
\hline 75 & sewilo_class & Colour classification from Sewiło et al. (2013) \\
\hline
\end{tabular}

Of the original list of 311 IRS staring-mode observations within the SAGE-SMC footprint, we discarded all 44 spectra for which we could not identify an IRAC point source within 3 arcsec in either the SAGE-SMC or the $\mathrm{S}^{3} \mathrm{MC}$ surveys. In cases where multiple matches were present within 3 arcsec, we manually compared the magnitudes with the flux levels of the spectra, and used the slit images to establish which source was responsible for the spectrum. We also consolidated duplicate measurements of the spectrum of a given source, as evidenced by their SAGE-SMC or $\mathrm{S}^{3} \mathrm{MC}$ identification, into a single entry in our analysis; this is sufficient for spectral identification purposes. This further reduced the number by 58 to a list of 209 unique Spitzer-IRAC point sources, with either SAGE-SMC or $\mathrm{S}^{3} \mathrm{MC}$ identifications, for which IRS staring-mode observations are available. We compiled all relevant information in a table available online. Table 2 describes the columns of the online table. Fig. 2 shows the distribution of the 209 sources over the SMC.
Preferring the IRAC coordinates over the spectrum coordinates, we then matched the IRAC point sources to a number of other infrared and optical photometric surveys. We obtained MIPS-[24], [70] and [160] matches, within a search radius of 3, 9, and 20 arcsec, respectively, from the SAGE-SMC survey (Gordon et al. 2011), corresponding to half a resolution element in these bands. We also searched the Wide-Field Infrared Survey Explorer (WISE) All-Sky Source Catalog for matches within 3 arcsec. We also searched for AKARI matches in the $\mathrm{N} 3, \mathrm{~N} 4, \mathrm{~S} 7, \mathrm{~S} 11, \mathrm{~L} 15$, and $\mathrm{S} 22$ bands within 3 arcsec using the catalogue provided by Ita et al. (2010). In the near-infrared, the Two Micron All Sky Survey (2MASS) Long Exposure $(6 \times)$ survey was searched for matches within 2 arcsec of the IRAC positions (SAGE-SMC matches with 2MASS), and we also used this search radius with the InfraRed Survey Facility (IRSF) catalogue (Kato et al. 2007). The Deep Near Infrared Survey (DENIS) of the Southern Sky catalogue (third release; Epchtein et al. 1999) 


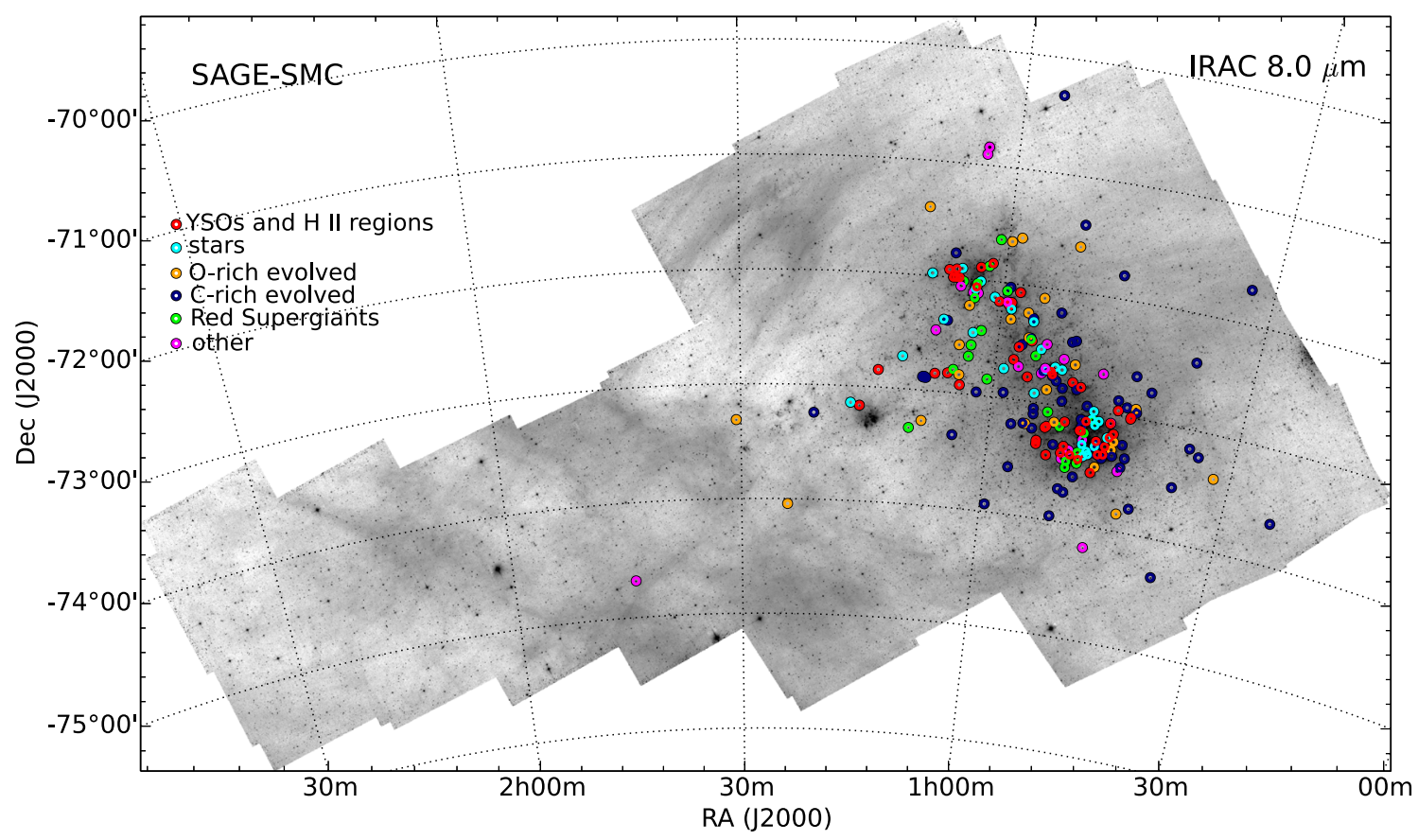

Figure 2. The SMC IRS targets distributed on the sky, overlaid upon a SAGE-SMC IRAC $8 \mu \mathrm{m}$ map. The colour of the points represents our classifications, according to the legend. All four YSO subcategories and the $\mathrm{H}$ II regions are grouped together in red. The class 'stars' contains objects classified as STAR and the subcategory of dusty OB stars. The 'O-rich evolved' category contains O-EAGB, O-AGB, O-PAGB and O-PN objects, and likewise the 'C-rich evolved' category groups together C-AGB, C-PAGB and C-PN objects. The RSGs are a group by themselves, and 'other' contains all other categories (see Table 3).

was also searched with a 2 arcsec search radius. In the optical, many of our sources have matches in the Magellanic Clouds Photometric Survey (MCPS; Zaritsky et al. 2002) and the catalogue published by Massey (2002). In both catalogues, we looked for matches within $1.5 \mathrm{arcsec}$ of the IRAC position. Some of the objects in our sample are actually too bright for those two optical surveys, and a search of the TYCHO catalogue with a radius of 3 arcsec filled in some of these gaps. All tabulated photometry is available from the online data base (see Table 2). We only provide the magnitudes for the purpose of evaluating the shape of the SED. Further information, including the photometric uncertainties, can be found in the respective source tables using the designations provided, as well as Appendix A.

\subsection{Bolometric magnitudes, variability, and colour classifications}

For each source bolometric magnitudes were calculated via a simple trapezoidal integration of the SED, to which a Wien tail was fitted to the short-wavelength data, and a Rayleigh-Jeans tail was fitted to the long-wavelength data. The following SED combinations were calculated:

(i) MCPS or Massey (2002) optical photometry; $J H K_{S}$ photometry; and IRAC and MIPS-[24] photometry, all as available (mbol_phot in Table 2);

(ii) like (i) but combined with the WISE photometry (mbol_phwi);

(iii) like (i) but combined with the IRS spectrum (mbol_phsp);

(iv) like (i) but combined with both the WISE photometry and the IRS spectrum (mbol_pwsp).
For sources where there is little reprocessing of the optical emission, i.e. little infrared excess, bolometric magnitudes were calculated using an SED fitting code (McDonald et al. 2009, 2012). This code performs a $\chi^{2}$-minimization between the observed SED (corrected for interstellar reddening) and a grid of BT-SETTL stellar atmosphere models (Allard, Homeier \& Freytag 2011), which are scaled in flux to derive a bolometric luminosity. This SED fitter only works effectively where a Rayleigh-Jeans tail is a good description of the 3-8 $\mu \mathrm{m}$ region, and provides a better fit to the optical and near-IR photometry than a Planck function. For the most enshrouded stars, fitting the SED with 'naked' stellar photosphere models leads to an underestimation of the temperature and luminosity, due to circumstellar reddening, and hence the integration method (above) for calculating $M_{\mathrm{bol}}$ is preferred for very dusty sources. Experience shows that good fits can be separated from bad fits in the NIR: if the model and observations differ at $I, J, H$ or $K_{S}$ by more than a magnitude in any band, the fit is considered bad. This retains the cases where the difference between model and observations in the MIR or FIR is large, but often in these cases the excess emission is unrelated to the point sources. In cases where it is related to the point source, making the source very red, the values calculated by trapezoidal integration provide a better estimate of $M_{\mathrm{bol}} . T_{\mathrm{eff}}, M_{\mathrm{bol}}$, and $L$ for the good fits are included in the online table as $t e f f \_m c d, m b o l \_m c d$, and $l u m \_m c d$, respectively (see Table 2).

The sample was then matched to the Optical Gravitational Lensing Experiment (OGLE-III) catalogue of long-period variables (LPV) in the SMC (Soszyński et al. 2011) and Groenewegen et al. (2009) to obtain variability periods, and the variability information is included in the online table (see Table 2). 
Finally, we included a number of colour classification schemes for comparison. First, Boyer et al. (2011) have extended the classification scheme developed by Cioni et al. (2006) to classify dusty mass-losing evolved stars into subcategories, using IRAC, MIPS, and NIR colours. We checked our source list against their catalogue for matches. Their classifications (O-AGB, C-AGB, xAGB, aO-AGB, RSG, RGB, and FIR) are included in the online table as boyer_class (see Table 2). Definitions of these classes can be found in Boyer et al. (2011). Furthermore, we also applied the colour classification scheme proposed by Matsuura et al. (2013) to the sources in our list. This classification scheme is also designed to distinguish between various kinds of very red objects, to estimate the dust production rate. We applied the cuts described by Matsuura et al. (2013, figs 4 and 5) on our sample and list the classifications that follow from these cuts (O-AGB, C-AGB, RSG) in our online table, as matsuura_class (see Table 2). The last colour classification scheme we apply is the one proposed by Sewiło et al. (2013) for YSOs, who applied classification cuts in the five different infrared CMDs, followed by visual inspection of images and SED fitting to select YSO candidates from the SAGE-SMC survey. We checked our source list against their catalogue and identified 'high-reliability' and 'probable' YSO candidates accordingly (sewilo_class; Table 2).

\section{THE CLASSIFICATION METHOD}

To classify our sample of 209 SMC point sources for which IRS staring-mode data exist, we follow the method described by Woods et al. (2011). Fig. 3 shows a restyled version of the classification decision tree. We made enhancements to the tree, which will be discussed in this section.

A literature search was performed for each object to retrieve other information useful in the process of classification, including (but not limited to) determination of stellar type, luminosity, age of nascent cluster of stars (if the object was found to be a member of a cluster), $\mathrm{H} \alpha$ detections, etc. This information was used in addition to the spectroscopic data, the photometric matches and derived bolometric luminosity, and the variability data, described in Section 2, to classify the sources. Any existing classification from the literature was used as a starting point before our spectral classification. Appendix A provides a brief summary of the literature survey for each object.

As in Woods et al. (2011), we adopt the following categories for our point source classification. Low- and intermediate-mass $\left(M<8 \mathrm{M}_{\odot}\right)$ post-main-sequence stars are classified by chemistry (O- or C-rich) and by evolutionary stage (AGB, post-AGB and planetary nebula - PN), hence our groupings O-AGB, O-PAGB, OPN, C-AGB, C-PAGB, C-PN. We propose an enhancement of the classification tree by Woods et al. (2011) to include early-type O-rich asymptotic giant branch (AGB) stars, namely O-EAGB. These stars do not show any evidence for dust features in their infrared spectra, but they do show long-period variability in OGLE and MACHO and some evidence for continuum infrared excess. Although these stars are in the early stages of AGB evolution, they are most likely more evolved than genuine early-AGB (E-AGB) stars, which have not yet started helium shell burning. E-AGB stars do not normally show long-period variability. We assume that O-EAGB stars are thermally pulsing AGB-type objects prior to the onset of, or just beginning, significant dust formation. More massive and luminous red supergiants (RSGs) have a class of their own, RSG. Young stellar objects (YSOs) can be classified phenomenologically into four groups, YSO-1, YSO-2, YSO-3, YSO-4 (see Woods et al. 2011 for the definition of these classes). Stars showing a stellar photosphere, but no additional dust or gas features, or long-period variability, are classified as STAR. One observational programme focused on stars showing a far-infrared excess in their MIPS photometry (Adams et al. 2013; Sheets et al. 2013), due to the illumination and heating of interstellar dust (the Pleiades effect); these objects are classified as a subcategory of STAR: dusty OB stars. The tree also distinguishes galaxies (GAL; even though none are actually found in this work) and $\mathrm{H}$ II regions ( $\mathrm{H}_{\mathrm{II}}$ ), and we furthermore have a class for $\mathrm{R}$ Coronae Borealis ( $\mathrm{R} \mathrm{CrB} \mathrm{stars;} \mathrm{labelled} \mathrm{RCRB} \mathrm{in} \mathrm{the} \mathrm{classification}$ table). Finally, the classification OTHER exists for objects of known type which do not belong in the categories above and do not follow from the classification tree (e.g. B[e] stars). The nature of these objects are usually identified by other means, and as such reported in the astronomical literature.

\subsection{Classification process}

Each source was classified independently by at least three of the co-authors. In cases where the classification was unanimous, it was simply adopted as the final classification, whereas in those cases where some discrepancies occurred, we asked additional co-authors to classify the sources, to settle the issue. In some cases, a discussion on the nature of the source ensued. We aimed to reach consensus among the co-authors on the nature of a source in cases where differences in classification occurred.

The lead author of this work (Paul Ruffle) developed an internal web browser-based classification tool, to facilitate the classification process. The decision tree was built into the tool as a series of questions, and the collected data were available in tabulated form. For each source the classification tool provided a slit image and plots of its spectrum, SED, log SED and bolometric magnitudes (see Fig. 1 for examples). Each author was free to use either the decision tree logic or their own method of classification. There was also room for the co-authors to add additional comments to the table. ${ }^{8}$

\section{SAGE-SPEC POINT SOURCE CLASSIFICATION}

Table 3 lists the classification types, used in the decision tree shown in Fig. 3, and counts for a total of 209 SMC point sources, for which Spitzer-IRS staring-mode observations are available. The classifications are also shown overplotted on the map of the SMC (Fig. 2).

Fig. 4 shows typical spectra of objects classified as one of the four YSO type objects, as well as a typical IRS spectrum of an $\mathrm{H}$ II region. The numbering 1-4 for the YSO classes represent an evolutionary sequence, with YSO-1 being the most embedded and YSO-4 the most evolved type of YSO, namely Herbig Ae Be (HAeBe) stars. This is evident from the spectral appearance of the

\footnotetext{
${ }^{8}$ Although we were able to use this tool to its conclusion and generate the final classification table prior to Paul Ruffle's unexpected death, the tool was intended to be available online indefinitely, for application on other data collections. Unfortunately, the internet service provider where this website was hosted recently stopped providing this service and as of yet, the coauthors have not been able to reconstruct and resurrect the website with the classification tool.
} 


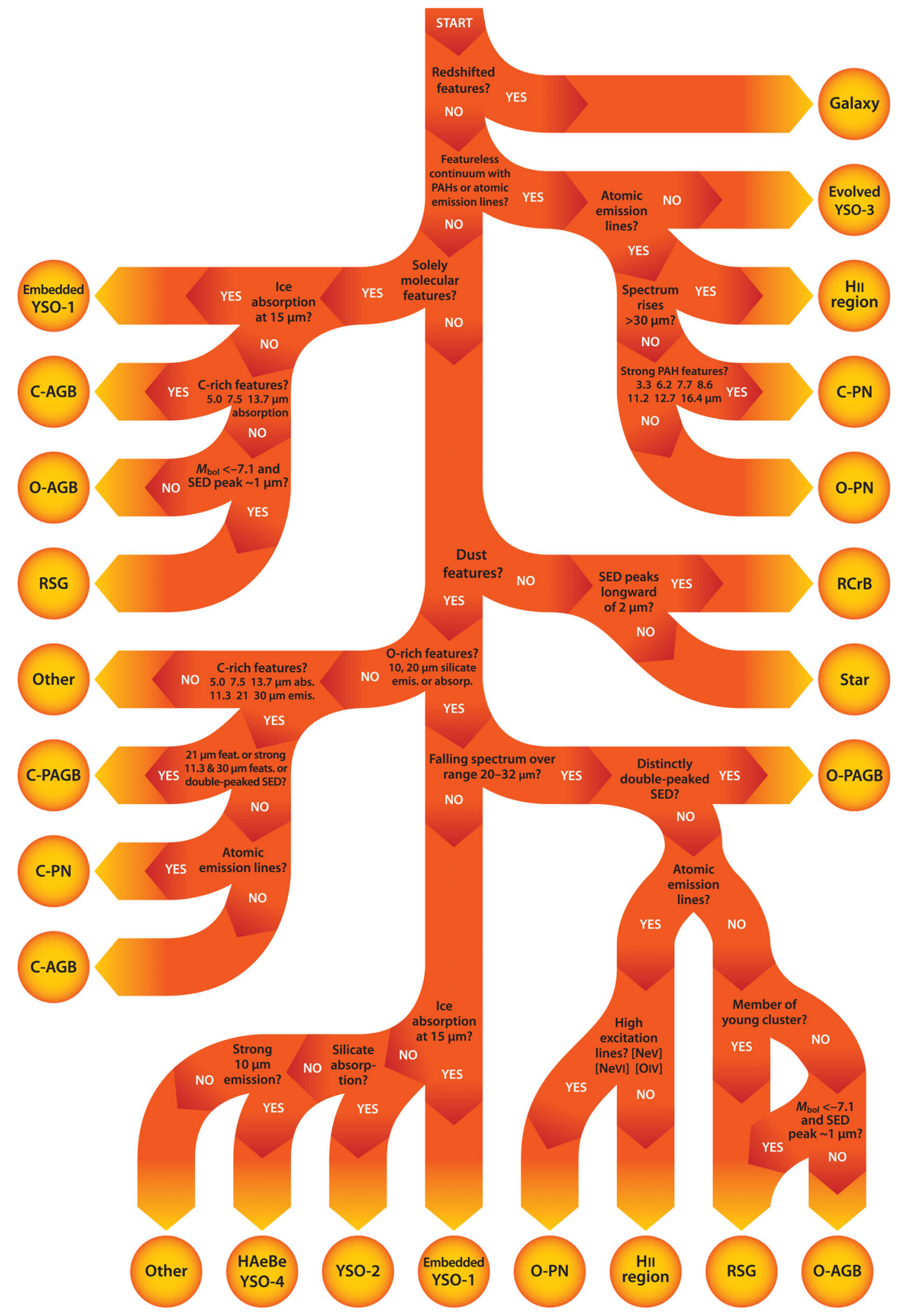

Figure 3. The logical steps of the classification decision tree, where Spitzer IRS spectra $(\lambda=5.2-38 \mu \mathrm{m})$, associated optical, NIR, WISE, IRAC and MIPS photometry, luminosity, variability, age and other information are used to classify SMC infrared point sources. See Table 3 for key to classification group codes. Figure restyled in appearance from the one published by Woods et al. (2011).

silicate feature, which appears in absorption towards YSO-1 objects, then gradually in self-absorption (YSO-2), until it finally appears in emission (YSO-3, YSO-4), for less embedded objects. The spectra of the YSO-1 objects also show ice absorption features, for instance the $\mathrm{CO}_{2}$ ice feature at $15.2 \mu \mathrm{m}$, further evidence of their early evolutionary phase. Polycyclic aromatic hydrocarbons (PAHs) are seen in the spectra of the YSO-3, YSO-4 and $\mathrm{H}$ II classes, indicative of a UV radiation field. Atomic lines are also seen, particularly 
Table 3. Classification types used in the decision tree shown in Fig. 3, and counts for a total of 209 SMC point sources. The last section of the table shows a breakdown of other known types (OTHER).

\begin{tabular}{lll}
\hline Code & \multicolumn{1}{c}{ Object type } & Count \\
\hline YSO-1 & Embedded YSOs & 14 \\
YSO-2 & YSOs & 5 \\
YSO-3 & Evolved YSOs & 22 \\
YSO-4 & HAeBe YSOs & 10 \\
H II & H II regions & 3 \\
O-EAGB & Early-type O-rich AGB stars & 8 \\
O-AGB & Oxygen-rich AGB stars & 11 \\
RSG & RSGs & 22 \\
O-PAGB & Oxygen-rich post-AGB stars & 1 \\
O-PN & Oxygen-rich PNe & 4 \\
C-AGB & Carbon-rich AGB stars & 39 \\
C-PAGB & Carbon-rich post-AGB stars & 3 \\
C-PN & Carbon-rich PNe & 20 \\
STAR & Stellar photospheres & 4 \\
& Dusty OB stars & 23 \\
RCRB & R CrB stars & 3 \\
BSG & Blue supergiant & 1 \\
WR & WR stars & 10 \\
OTHER & B[e] stars & 2 \\
& Foreground stars & 2 \\
& S stars & 1 \\
& Symbiotic stars & 1 \\
\hline & &
\end{tabular}

in YSO-3 and $\mathrm{H}$ II objects, and the latter category shows a rising continuum indicative of cold dust in the vicinity of the ionizing star.

Fig. 5 shows typical spectra in the group of oxygen-rich evolved stars. The earliest type of O-rich AGB stars are shown at the bottom of the plot (O-EAGB), and the spectra shown here do not show any dust features, while the signature of oxygen-rich molecular species may be present in the spectra. A slight change of slope due to a small infrared excess caused by thermal dust emission, may be visible in the SED, however. Later type O-AGB stars, RSGs and oxygen-rich post-AGB stars (O-PAGB), share spectroscopic characteristics, such as the presence of silicate emission features, although the detailed shape can be different. To distinguish between the RSG and OAGB category, a bolometric luminosity cut of $M_{\mathrm{bol}}=-7.1$ is used, while the distinction between O-AGB and O-PAGB is based on the presence of a detached shell where a double-peaked SED is used as a criterion for the latter category. This is demonstrated in the lower-right panel of Fig. 6 showing the SED of the sole O-PAGB object in the sample, LHA 115-S 38 (SMC IRS 257). O-rich PNe (O-PN) may still show a distinguishable oxygen-rich chemistry in their dust mineralogy (although not in the case shown), but they are discriminated from C-PN using the presence of PAH lines in the spectra of the carbon-rich objects.

Fig. 7 gives an overview of the 5-38 $\mu \mathrm{m}$ spectral appearance of carbon-rich evolved stars. Tracers of the carbon-rich chemistry are the $\mathrm{C}_{2} \mathrm{H}_{2}$ molecular absorption bands at 5.0, 7.5, and $13.7 \mu \mathrm{m}$, the $\mathrm{SiC}$ dust feature at $11.3 \mu \mathrm{m}$, the $21-\mu \mathrm{m}$ feature (which remains unidentified and really peaks at $20.1 \mu \mathrm{m}$ ), and the so-called $30-\mu \mathrm{m}$ feature, which has recently been suggested to be due to the same carbonaceous compound that carries the continuum (Otsuka et al. 2014), while the MgS identification is under debate (Zhang, Jiang \& Li 2009; Lombaert et al. 2012). Again, the distinction between the C-PAGB and C-AGB categories is based on whether the SED is double-peaked, which is evidence for a detached shell, indicating

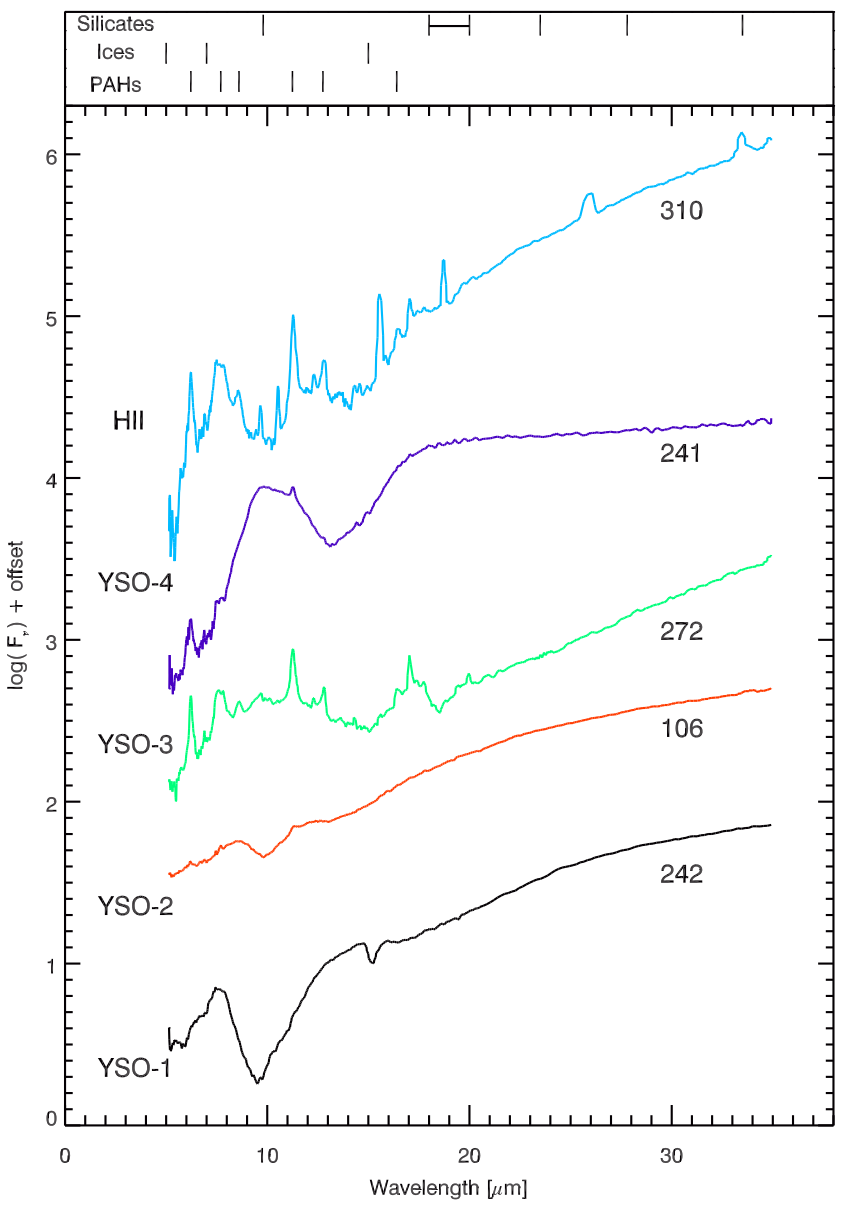

Figure 4. Example spectra in the $\mathrm{YSO}$ and $\mathrm{H}$ II categories. From bottom to top, we show examples of YSO-1 through 4, and a spectrum of an $\mathrm{H}$ II region, in an evolutionary sequence from young to more evolved. The spectra are labelled with their SMC IRS number. Discernible spectral features are indicated with tick marks and labels at the top of the diagram. The heavily embedded YSO-1 spectrum shows silicates and ices in absorption. The YSO-2 spectrum shows silicate in self-absorption. Silicate emission and $\mathrm{PAH}$ features are visible in the spectrum of the YSO-3, YSO-4, and $\mathrm{H}$ II objects, albeit in different ratios. The $\mathrm{H}$ in region has a rising SED slope.

mass-loss has stopped. Fig. 6 shows two clear examples of this, namely SMC IRS 243 and SMC IRS 268. SMC IRS 95 is confirmed to be a C-PAGB star (Kraemer et al. 2006; van Loon et al. 2008), despite its absence of a double-peaked SED. C-PAGB and C-PN also show the UV-excited PAH features, and in case of the C-PN, the presence of atomic emission lines.

Fig. 8 shows typical spectra of a number of star-like categories, namely (from top to bottom) stellar photospheres, with no discernible dust features in the spectrum; R CrB stars, which only appear to show a dust continuum with no spectral substructure; a Blue Supergiant in our sample, which appears to have a strong far-infrared excess on top of a stellar photosphere, and finally WolfRayet (WR) stars, which may form dust and show the corresponding infrared excess in the spectrum. The Blue Supergiant and the WR star classifications are taken from the literature, and are not based on the infrared spectroscopy. Similarly, Fig. 9 shows the spectra of the object types group under OTHER, of which the classifications are taken from the literature (see Appendix A). The examples shown in Fig. 9 represent from top to bottom a $\mathrm{B}[\mathrm{e}]$ star, a foreground AGB 


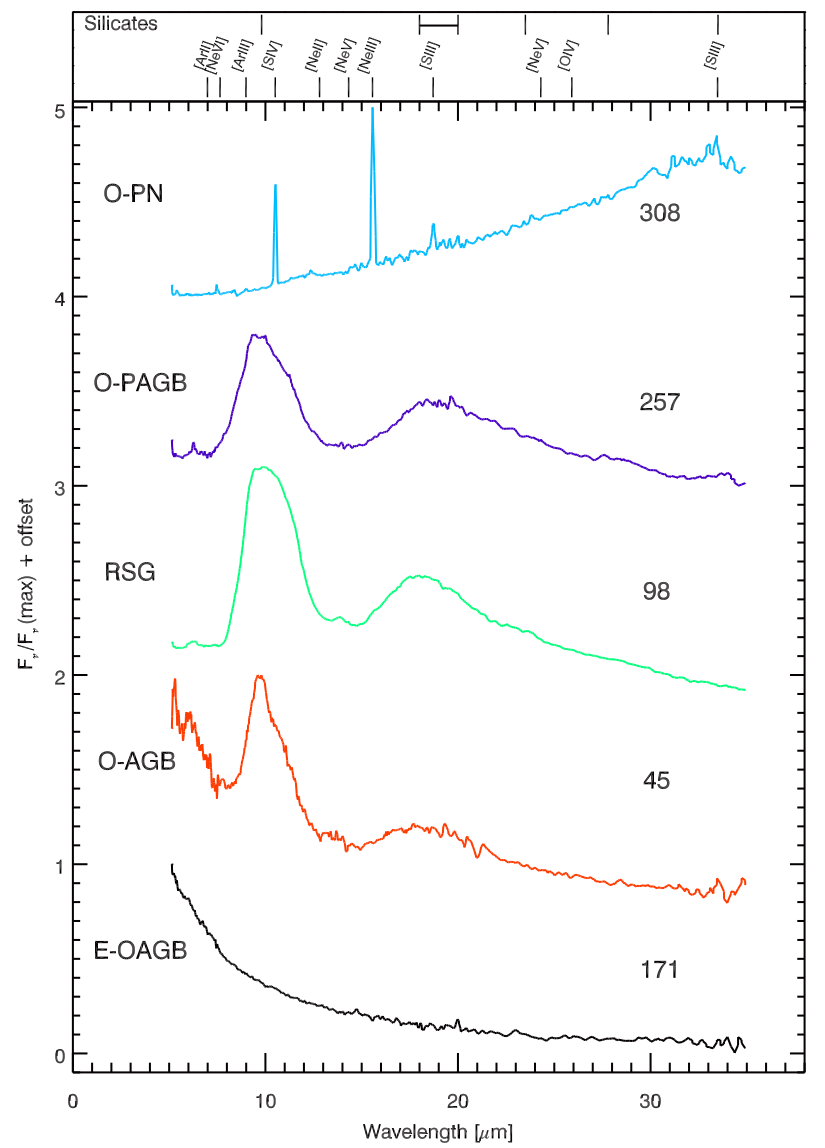

Figure 5. Example spectra of different types of O-rich evolved stars. The spectra are labelled with their SMC IRS number. At the top, the wavelengths of the silicate features and spectral lines are labelled. At the bottom of the plot, the spectrum of the E-OAGB star closely resembles a stellar photosphere. The spectra of the O-AGB, RSG, and O-PAGB star all show the silicate emission features. Distinguishing between these three types is not possible from IRS spectroscopy alone, and additional information on SED shape and bolometric luminosity is required. The top spectrum is characteristic of an O-PN.

star, an S star and a symbiotic star. Their infrared spectra are not used to achieve this classification, and the IRS data are plotted only for illustration.

Figs 10-12 show the 209 classified point sources on the [8.0] versus $J-[8.0] \mathrm{CMD}$ and two different colour-colour diagrams (CCDs), overlayed on the SAGE-SMC PSC (Gordon et al. 2011) in grey-scale.

The [8.0] versus $J-$ [8.0] CMD (Fig. 10) shows a large spread for the population of 209 objects. The stellar atmospheres (STAR) and WR stars have colours more or less indistinguishable from the bulk of the SAGE-SMC catalogue (with $J-[8.0] \sim 0-1 \mathrm{mag}$ ), and modest brightness at $8.0 \mu \mathrm{m}$, even though these stars are amongst the brightest stars in the optical in the SMC. All other categories displayed are bright in the IRAC [8.0] band, and often show considerable redness in their $J-[8.0]$ colour. In this diagram RSG, $\mathrm{O}-\mathrm{AGB} / \mathrm{O}-\mathrm{EAGB}, \mathrm{C}-\mathrm{AGB}$, and YSOs (all classes combined) are reasonably well separated from each other, although there are some interlopers. It appears to be difficult to separate PNe and YSOs on the one hand, and the most extreme C-AGB stars and YSOs on the other hand. Distinguishing between the four YSO classes is also not possible in this diagram.

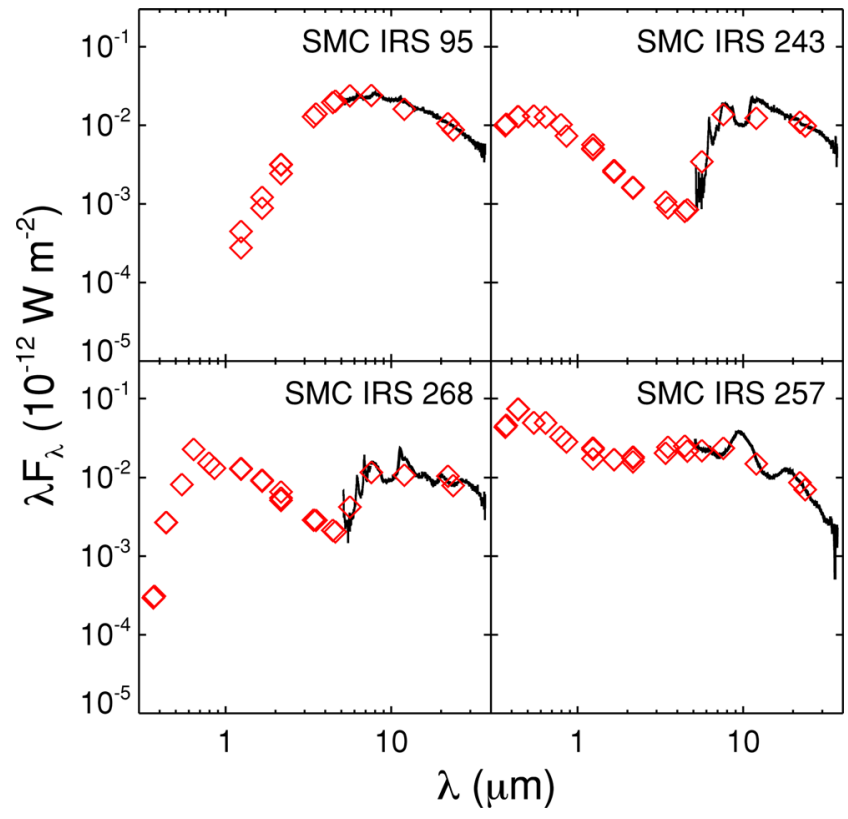

Figure 6. SEDs of the four post-AGB stars in the sample. The SMC IRS spectra 95, 243 and 268 correspond to C-PAGB objects, while SMC IRS 257 is the spectrum of a O-PAGB object. The black lines represent the IRS spectra, while the red diamonds correspond to the collected photometric measurements for each source. The double-peaked structure used as a distinguishing feature is visible in the SEDs of 243, 268 and 257. 2MASS J00364631-7331351 (SMC IRS 95) does not show a double peaked structure, but its C-PAGB nature is confirmed using near-infrared spectroscopy and the PAH features in the IRS spectra (See Appendix A).

Fig. 11 is a CCD composed of the four IRAC bands, namely the [3.6]-[4.5] versus [5.8]-[8.0] colours. The advantage of using a CCD is that it is distance independent. The coverage of the sample of 209 objects fans out nicely over colour-colour space. In this diagram, the stars and WR stars no longer separate well from the rest of the sample, however it now appears easier to separate YSOs from $\mathrm{C}$-AGB stars on the one hand and $\mathrm{PNe}$ on the other hand. However, C-PAGB and O-PAGB stars probably overlap with the colour-colour space taken up by YSOs, as they transition from the AGB region to the PN region in the diagram. But as this phase is short-lived, pollution of the colour-selected YSO sample with postAGB stars is limited. Subdivision within the YSO category is still not possible, and also the O-(E)AGB objects do not seem to occupy a unique part of colour-colour space.

Finally, Fig. 12 shows the $J-K_{S}$ versus [8.0]-[24] CCD. In this $\mathrm{CCD}$, the C-AGB objects are easily separated from all other types of objects, as their $J-K_{S}$ colour quickly increases, with increasing [8.0]-[24]. For O-(E)AGB stars and RSGs this increase is less steep, forming a separate branch in the middle of the plot. O-PN and C-PN group together with YSOs towards the top of the diagram, showing the highest values of [8.0]-[24], against modest $J-K_{S}$ reddening.

\section{COMPARISON WITH EXISTING COLOUR CLASSIFICATIONS}

These 209 spectral classifications will allow us to verify existing infrared photometric classification schemes that have come out of recent studies of the Magellanic Clouds. We compare our results with three distinct colour classification schemes: (a) the $J H K_{S}$ colour classification scheme for evolved stars by Cioni et al. (2006), 


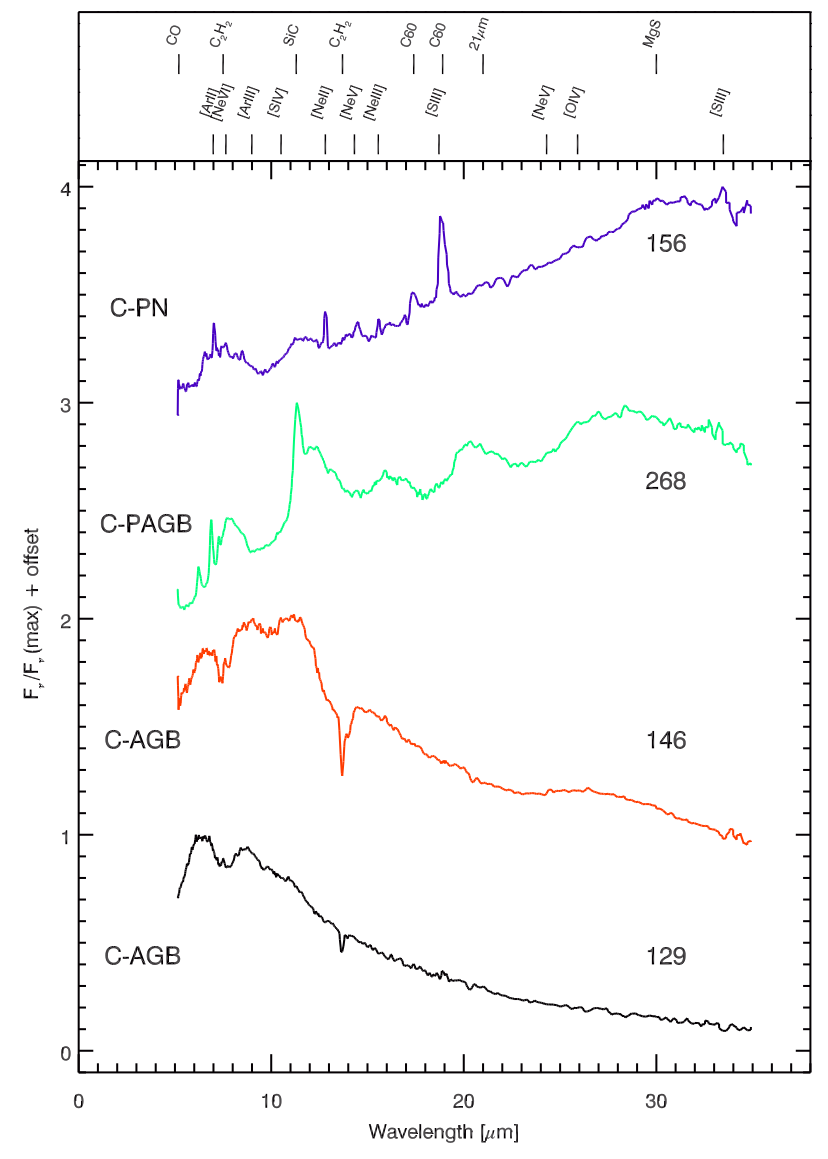

Figure 7. Example spectra of C-rich evolved stars. From bottom to top two C-AGB stars are shown, followed by a C-PAGB and a C-PN. All four spectra are labelled with their SMC IRS number. The tick marks at the top show the position of characteristic spectral features. In the C-AGB spectra, the molecular absorption bands of $\mathrm{C}_{2} \mathrm{H}_{2}$ are visible, as well as emission due to $\mathrm{SiC}$ and possibly $\mathrm{MgS}(30 \mu \mathrm{m}$, not in all sources). The C-PAGB object no longer shows the molecular absorption bands, but the $\mathrm{SiC}$ and the $\mathrm{MgS}$ (not always) are still visible. Other features include PAH bands and the 21- $\mathrm{mm}$ feature. The infrared spectrum of C-PN objects shows the spectral features due to PAHs and atomic lines.

expanded by Boyer et al. (2011) to include mid-IR wavelengths; (b) the IRAC classification scheme for AGB stars and RSGs by Matsuura et al. (2013), which is based on the previous work by Matsuura et al. (2009) on the LMC; and (c) the Spitzer classification scheme to select YSO candidates by Sewiło et al. (2013), based on earlier work by Whitney et al. (2008).

\subsection{Boyer et al. (2011)}

In order to classify all evolved stars in the SAGE-SMC (Gordon et al. 2011) data, Boyer et al. (2011) devised a classification scheme, based on the 2MASS classification scheme presented by Cioni et al. (2006). The basis for this classification scheme is the $K$ versus $J$ $-K_{S}$ CMD (Fig. 13), showing the cuts for the RSG, O-AGB, and C-AGB object classes, superposed on the SAGE-SMC point sources (grey pixels). The dotted line refers to the tip of the red giant branch (RGB), and Boyer et al. (2011) use this line to separate RGB stars from the AGB and RSG categories. Photometrically classified objects from Boyer et al. (2011) are shown as coloured pixels. At the red end of the diagram, some photometrically classified C-AGB stars appear below the diagonal cut, or even below the

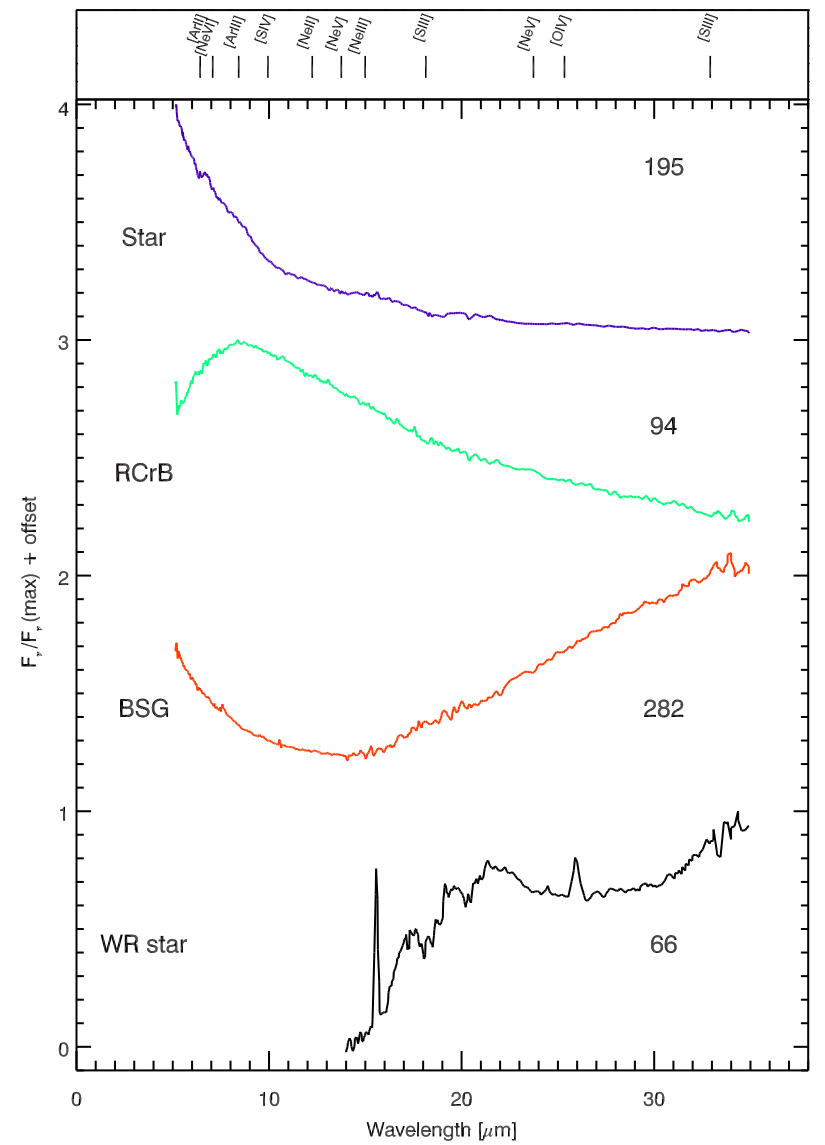

Figure 8. Example spectra of objects with stellar photospheres or similar. Shown here from bottom to top are the spectra of a WR star, a BSG, a R CrB star and a regular stellar photosphere, labelled with their SMC IRS number. The WR star shows some atomic lines in its spectrum (marked at the top of the diagram), but the other spectra are rather featureless. The infrared excess in the $\mathrm{R} \mathrm{CrB}$ and BSG objects are caused by dust emission.

dotted line corresponding to the tip of the RGB. These objects are called extreme AGB stars, predominantly carbon-rich, which are defined as $J-[3.6]>3.1 \mathrm{mag}$ (e.g. Blum et al. 2006). Often these objects are so red, that they are not detected in 2MASS $J H K_{S}$, and alternative selection criteria in the mid-infrared are required. The reader is referred to Boyer et al. (2011) for a detailed description. The larger symbols in Fig. 13 represent the sample of Spitzer-IRS spectroscopically classified objects described in this work.

\subsubsection{C-AGB stars}

Objects showing the C-AGB spectral signature are all classified as either C-AGB or x-AGB ( most of which are expected to be C-rich), according to the Boyer et al. (2011) classification, although three of these objects are not included in the catalogue published by Boyer et al. (2011). These objects (OGLE SMCLPV-7488 (SMC IRS 44; SSTISAGEMA J004903.78-730520.1); 2MASS J01060330-7222322 (SMC IRS 109; SSTISAGEMA J010603.27-722232.1); and 2MASS J00561639-7216413 (SMC IRS 129; SSTISAGEMA J005616.36-721641.3)) are among a larger set of objects that had not been properly matched between the IRAC Epochs in the mosaicked catalogue (Srinivasan et al. in preparation). These objects were thus missing photometric measurements in the point source catalogue, in some bands, and were 


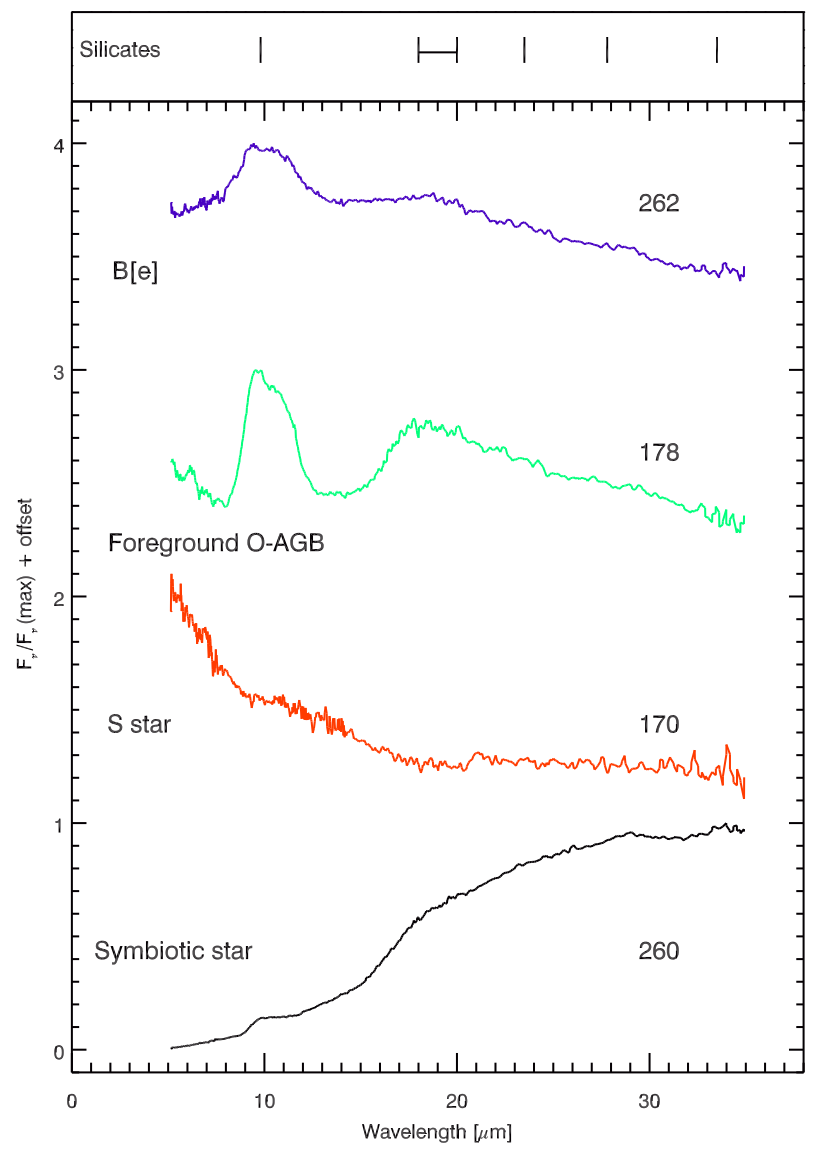

Figure 9. Example IRS spectra of objects in the OTHER category. From bottom to top we show the spectra of a symbiotic star, an $\mathrm{S}$ star, a foreground (Galactic) O-AGB star and a B[e] star, all labelled with their SMC IRS number. Relevant spectral features due to silicates are labelled at the top of the figure.

therefore not classified (correctly) by Boyer et al. (2011). The online table described by Table 2 shows the correct photometry for these three targets.

\subsubsection{Red supergiants}

Most of the 21 spectroscopically classified RSGs indeed fall within the RSG strip defined by Boyer et al. (2011). Only three of the objects classified as an RSG by us were classified differently by Boyer et al. (2011) using only the photometry: HV 11417 (SMC IRS 115) is designated as an FIR object by Boyer et al. (2011) and Massey SMC 55188 (SMC IRS 232) as an O-AGB star; and IRAS F00483-7347 (SMC IRS 98) as an x-AGB star. HV 11417 is a variable star with a period of $1092 \mathrm{~d}$, and an amplitude in the $I$ band of 1.9 mag (Soszyński et al. 2011). Due to this large amplitude, the timing of the observations affects the IR colours of the object significantly, which is why the photometry measurements show a small positive slope between 8 and $24 \mu \mathrm{m}$ ([8.0]-[24] $>2.39 \mathrm{mag})$, causing it to be classified as an FIR object by Boyer et al. (2011), while the slope of the IRS spectrum is distinctly negative. If we disregard the FIR colour cut, which is meant to separate AGB stars from objects such as YSOs, PNe and background galaxies, this object would have been classified as an O-AGB star, which deviates from our determination of RSG, and from past classifications (Elias, Frogel \& Humphreys 1980). The bolometric luminosity of this object is close to the RSG/O-AGB boundary, and could be affected by the variability of the object too. The large amplitude favours a classification as luminous AGB star over an RSG. RSGs were separated from O-AGB stars using the classical AGB bolometric magnitude limit (Fig. 3), but this boundary is not absolute. AGB stars undergoing hot bottom burning (HBB) can be brighter than this limit, while less-evolved RSGs can be fainter. This causes some disagreement in classifications of stars near the boundary.

Alternatively, the luminosity boundary between RSG and O-AGB may not be properly represented by the cuts in the $K_{S}$ versus $J-K_{S}$ diagram from Boyer et al. (2011). Similarly, the misclassification of Massey SMC 55188 also suggests that the bolometric cut we applied to distinguish between O-AGB stars and RSGs does not correspond to the boundaries between these two categories in the $K_{S}$ versus $J-K_{S}$ CMD. IRAS F00483-7347 clearly shows an oxygen-rich chemistry in its spectrum, with the presence of the amorphous silicate bands at 9.7 and $18 \mu \mathrm{m}$. The object is heavily embedded, with a very red SED, and the $9.7-\mu \mathrm{m}$ feature starts to show signs of self-absorption. IRAS F00483-7347 demonstrates that not all x-AGB objects are in fact carbon-rich AGB stars.

\subsubsection{O-AGB stars}

All objects classified as O-EAGB in this work are similarly classified as O-AGB by Boyer et al. (2011). The spectrally confirmed O-AGB objects, however, show a much wider spread in colourmagnitude space than the defined strip, and they encroach on the photometrically defined RSG, C-AGB and FIR categories. In particular, only two spectroscopically classified O-AGB stars, HV 12149 (SMC IRS 45) and 2MASS J00444463-7314076 (SMC IRS 259) are classified as O-AGB based on photometry. One object (HV 1375; SMC IRS 110) is classified as a C-AGB based on its photometric colours, and a further six objects (RAW 631, 2MASS J00463159-7328464, 2MASS J00445256-7318258, BMB-B 75, IRAS F01066-7332 and HV 12956) are apparently sufficiently red ([8.0]-[24] > $2.39 \mathrm{mag}$ ) to be classified as FIR, even though they are not background galaxies, YSOs or PNe. These six objects are heavily embedded, and show the effect of a high optical depth in the relative strength of the $18 \mu \mathrm{m}$ silicate band with respect to the 9.7- $\mu \mathrm{m}$ band. They also show evidence for the presence of crystalline silicates in their spectrum, another sign of high optical depth (Kemper et al. 2001; Jones et al. 2012). Finally, HV 2232 (SMC IRS 230) and HV 11464 (SMC IRS 309) are photometrically classified as RSGs (Boyer et al. 2011, see the table described by Table 2).

\subsubsection{Additional sources}

The further nine interlopers in the $\mathrm{C}-\mathrm{AGB} / \mathrm{x}-\mathrm{AGB}$ section of the CMD defined by Boyer et al. (2011) are a mixed bag of objects, all but one falling in the $\mathrm{x}$-AGB category. These objects reflect selection biases and include rare categories of previously known types such as R CrB stars (2MASS J00461632-7411135, 2MASS J00571814-7242352 and OGLE SMC-SC10 107856), an S star (BFM 1) and a B[e] star (Lin 250). These object types are not included in the Boyer et al. (2011) classification scheme, and could therefore never have agreed with the spectral classification. True misclassifications are the objects thought to be C-rich AGB stars, only to be revealed to be something else based on their IRS spectroscopy. These include O-PAGB star LHA 115-S 38, YSO-2 object 2MASS J01050732-7159427, and RSG IRAS F00483-7347. NGC 346:KWBBe 200 is a special case, as it was thought to be a $\mathrm{B}$ [e] supergiant (Wisniewski et al. 2007a), but its classification has 


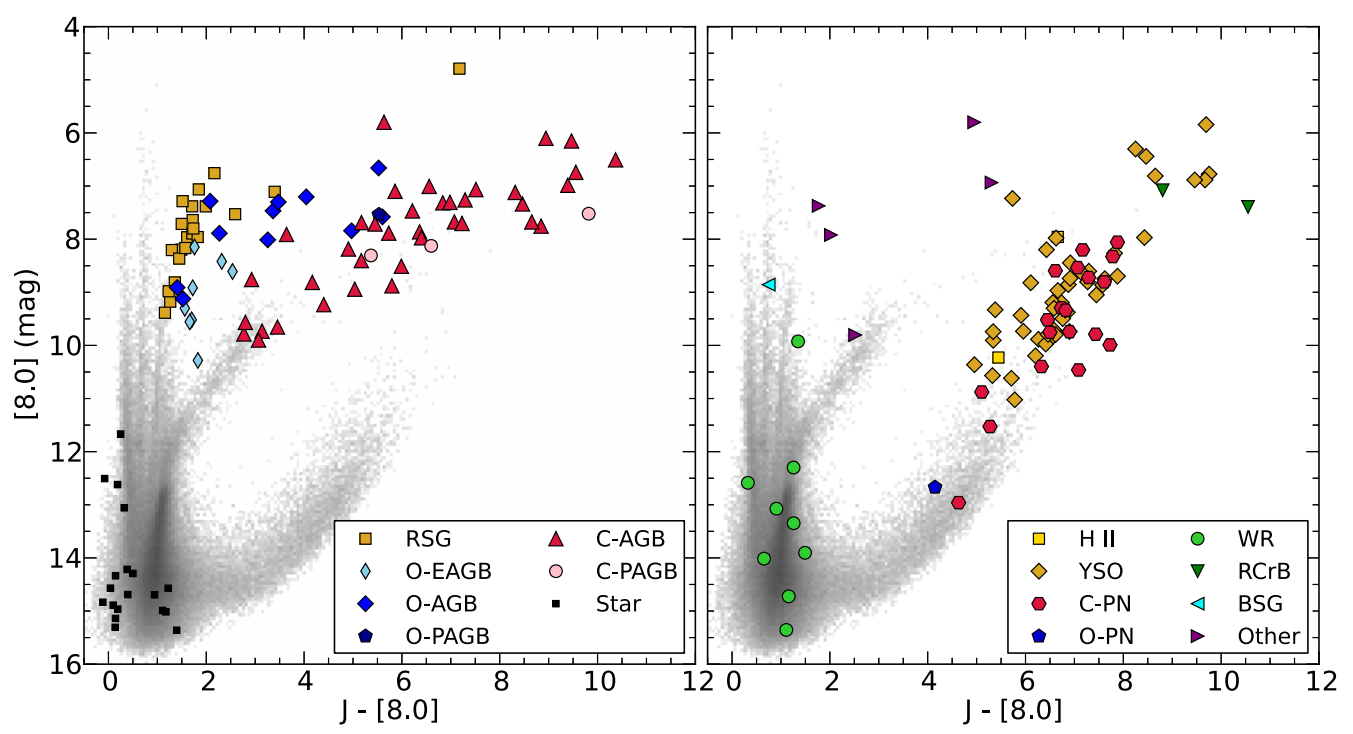

Figure 10. [8.0] versus $J-[8.0]$ CMD. The grey-scale in the background represents a density plot of the SAGE-SMC PSC, while the coloured symbols are the sources classified in this work. For clarity, the plot is duplicated with the coloured symbols spread out over the left- and right-hand plot according to the legend.

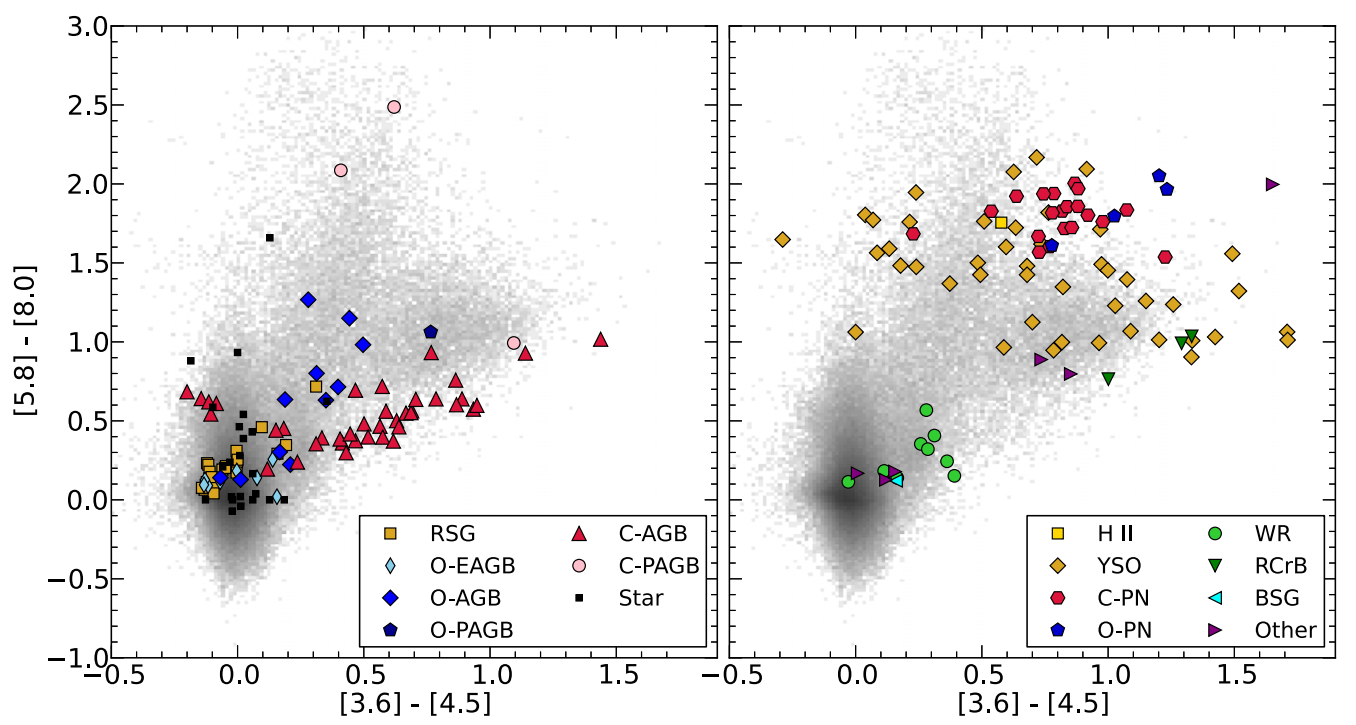

Figure 11. [5.8]-[8.0] versus [3.6]-[4.5] all-IRAC CCD. Description as in Fig. 10.

recently been revised to a YSO (Whelan et al. 2013), in line with our classification of YSO-3 (See Appendix A).

The FIR category defined by Boyer et al. (2011) was introduced to exclude YSOs, compact $\mathrm{H}$ II regions, $\mathrm{PNe}$ and background galaxies from the AGB/RSG sample, by applying the [8]-[24] > 2.39 mag cut, corresponding to a rising continuum. Indeed, 14 out of 23 FIR objects are either C-PN (three objects) or YSOs (11 objects), according to their IRS spectra. The remaining nine FIR objects include the six O-AGB stars discussed in Section 5.1.3, but also a symbiotic star (SSTISAGEMA J005419.21-722909.7; SMC IRS 260) and a C-PAGB (2MASS J00364631-7331351; SMC IRS 95), both of which are classified based on their IRS spectra.

\subsection{Matsuura et al. (2013)}

The second classification scheme was proposed by Matsuura et al. (2009) to identify mass-losing O-rich and C-rich AGB stars, as well as RSGs, in order to estimate the dust production budget in the LMC, separated out by C-rich and O-rich chemistries. The scheme is based on the IRAC bands, using the [8.0] versus [3.6]-[8.0] CMD, and separates out the O-rich AGB stars and RSGs on the one hand, and C-rich AGB stars on the other hand (Fig. 14). Matsuura et al. (2009) used only this diagram to classify the IRAC point sources in the LMC, but for the SMC, Matsuura et al. (2013) added an extra step to overcome pollution between the categories for redder [3.6]-[8.0] sources. Indeed, from Fig. 14 it is clear that the red part of the C-AGB section is dominated by sources identified as YSOs (tan diamonds), while a significant fraction of the O-AGB objects (blue diamonds) also fall within the C-AGB section. In the second step, MIPS and NIR data are included, and a complex series of cuts is made in the $K_{S}-$ [24] versus $K_{S}-$ [8.0] CCD (see fig. 5 in Matsuura et al. 2013, the equations are not provided). In cases where the classification using the $K_{S}-[24]$ versus $K_{S}-[8.0]$ diagram (Fig. 15) deviates from the [8.0] versus [3.6]-[8.0] classification, 

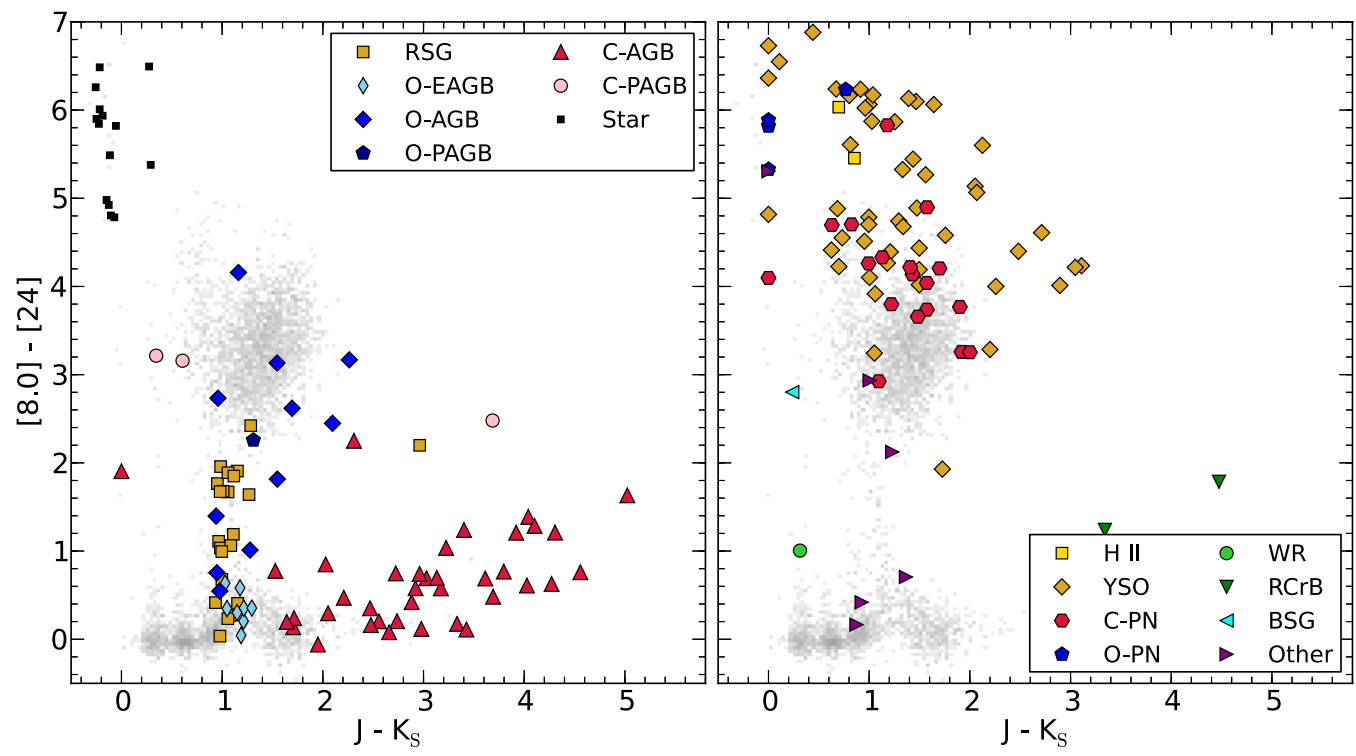

Figure 12. [8.0]-[24] versus $J-K_{S}$ CCD. Description as in Fig. 10.
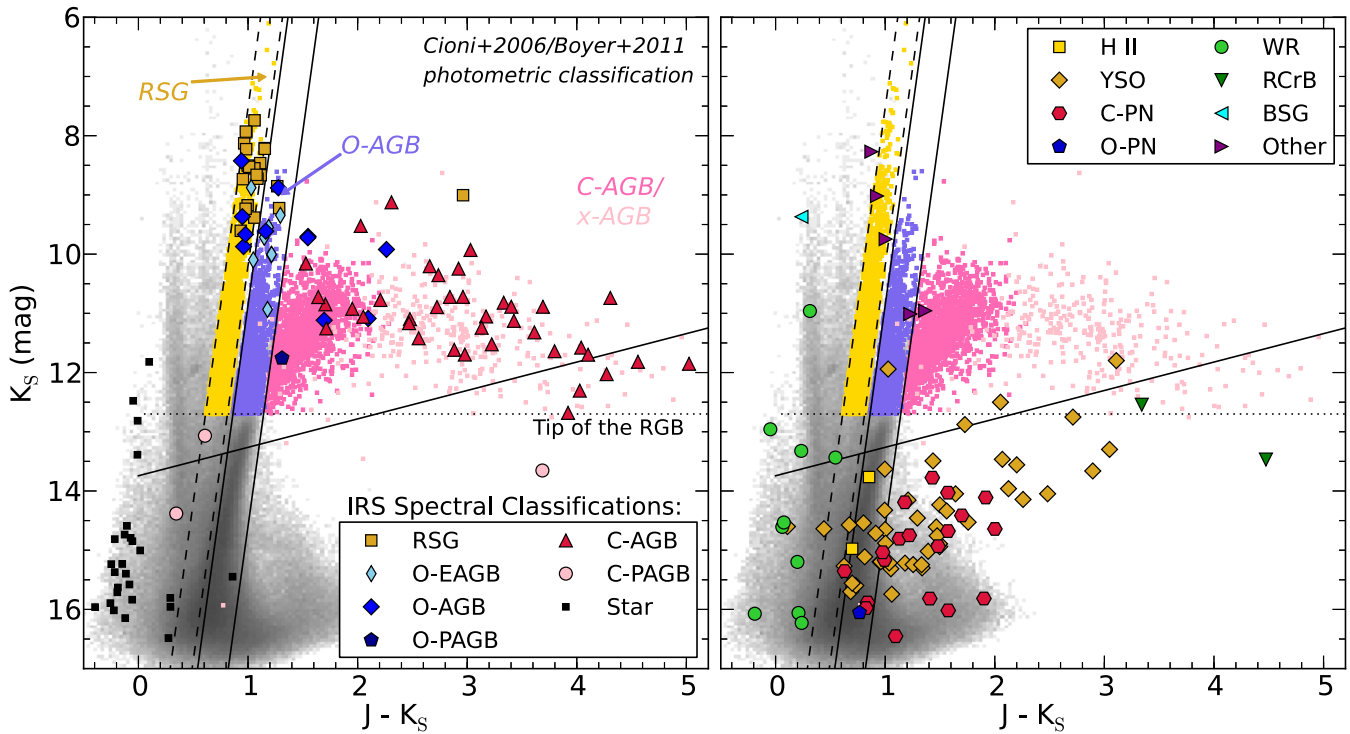

Figure 13. The Boyer et al. (2011) photometric classification, based on the work by Cioni et al. (2006), applied to the SMC. Boyer et al. (2011) begin with the $J-K_{S}$ classification shown here, then use 3.6 and 8 - $\mu$ m photometry to recover and classify the dustiest sources. The slanted lines in this $K_{S}$ versus $J-K_{S}$ CMD represent the boundaries of the C-AGB, O-AGB, and RSG categories, and the horizontal dotted line shows the boundary between the tip of the RGB and the earlier type AGB stars. In grey-scale, the SAGE-SMC PSC is shown in the form of a density plot. The lilac, yellow and dark pink dots represent the RSG, O-AGB and C-AGB objects, as they are classified in the $K_{S}$ versus $J-K_{S}$ CMD. The light pink dots are the x-AGB stars, selected by Boyer et al. (2011), replacing any $K_{S}$ versus $J-K_{S}$ classification. The x-AGB objects fall mostly within the C-AGB boundaries. The coloured symbols represent the objects spectrally classified in this work, following the legends and spread out over two panels for clarity.

the $K_{S}-[24]$ versus $K_{S}-[8.0]$ classification takes preference. Most spectral classifications agree with the photometric classification in the second step (see Fig. 15). In case where sources were only classified in one of the two steps, we used that classification. We have executed this classification method for our 209 sources, and included the results in the table described by Table 2 .

\subsubsection{Carbon-rich AGB stars}

The two-step classification described by Matsuura et al. (2013) correctly identifies most of the spectroscopically classified C-AGB stars in our sample as such. In only three cases was an object pho- tometrically classified as being oxygen-rich (RSG/O-AGB), while the spectroscopy shows the carbon-rich nature of the source. These sources are 2MASS J00515018-7250496 (SMC IRS 103); 2MASS J00524017-7247276 (SMC IRS 127) and IRAS 00350-7436 (SMC IRS 238). IRAS 00350-7436 is among the brightest midinfrared objects in the SMC, and falls above the C-AGB cut in fig. 4 of Matsuura et al. (2013), due to its exceptional brightness.

Furthermore, the classification of several C-AGB stars by Matsuura et al. (2013) disagrees with the spectral classification. These include: a C-rich post-AGB star (2MASS J00364631-7331351; SMC IRS 95), four O-rich early-type AGB stars (HV 1366, HV 11303, HV 838, and HV 12122; SMC IRS 36, 38, 39, and 116, 

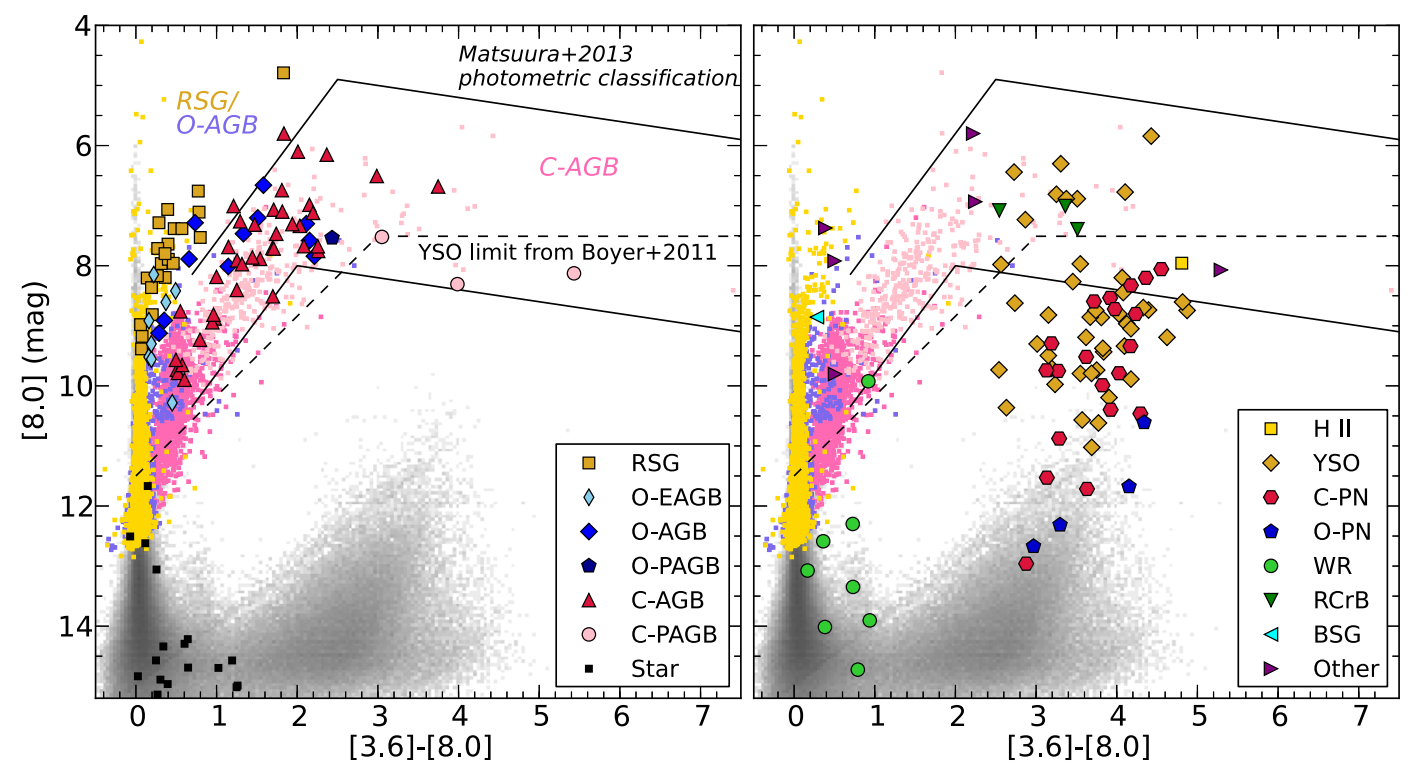

Figure 14. First step of the Matsuura et al. (2013) photometric classification method, based on the work by Matsuura et al. (2009), applied to the SMC. The solid lines in this [8.0] versus [3.6]-[8.0] CMD represent the boundaries of the C-AGB category, and provide a lower boundary to the RSG/O-AGB category. The dashed line shows the position of the YSO limit used by Boyer et al. (2011). All colours have the same meaning as in Fig. 13.

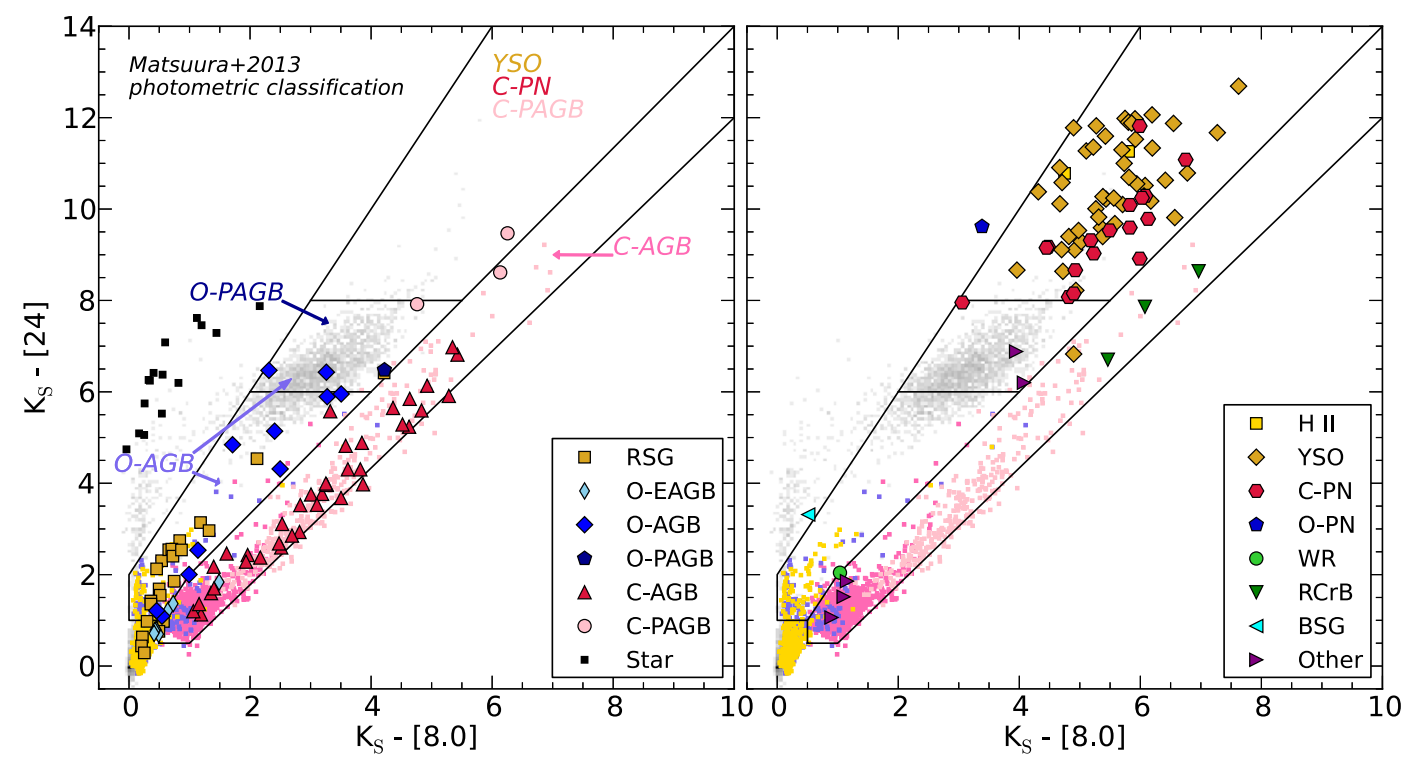

Figure 15. Second step of the Matsuura et al. (2013) photometric classification method. The solid lines in this $K_{S}-$ [24] versus $K_{S}-[8.0]$ CCD represent the boundaries of the various evolved star categories. All colours have the same meaning as in Fig. 13.

respectively), a WR star (HD 5980; SMC IRS 281), an RSG (HV 11262; SMC IRS 111), a YSO-3 object (NGC 346:KWBBe 200; SMC IRS 19), the three R CrB stars (2MASS J00461632-7411135, 2MASS J00571814-7242352, and OGLE SMC-SC10 107856; SMC IRS 94, 114, and 245, respectively), the two foreground Orich AGB stars NGC 362 SAW V16 and HV 206, the S star BFM 1 and the symbiotic star SSTISAGEMA J005419.21-722909.7. The last four objects are all in the OTHER category, which contains subclasses the work by Matsuura et al. (2013) does not seek to classify. The four O-rich early-type objects have actually long been recognized as such, in some cases their nature was already known in the 1980 s, and they fall only slightly outside the boundaries in fig. 5 of Matsuura et al. (2013). The RSG HV 11262 has very similar $K_{S}-$ [8.0] and $K_{S}-[24]$ colours to the four O-EAGB objects. 2MASS
J00364631-7331351 is not properly filtered out by Fig. 5, as it falls just below the $K_{S}-[24]=8$ mag cutoff that is meant to exclude YSO, C-PN, and C-PAGB objects from the C-AGB category. The R $\mathrm{CrB}$ stars represent a rare class which, not surprisingly, overlaps in infrared colours with the C-AGB stars, as it is believed that the dust in these stars is carbonaceous (Feast 1986). HD 5980 is the only WR star that is classified by Matsuura et al. (2013). Although other WR stars do appear in Fig. 14, they do not receive a classification as they are too faint in the [8.0] band. HD 5980 is considerably brighter in the [8.0], and is the only WR star with a MIPS-[24] detection in the SMC (Bonanos et al. 2010). Closer inspection of the IRS spectrum seems to suggest that a chance superposition with a compact $\mathrm{H}$ II region gives rise to this $24 \mu \mathrm{m}$ detection, and is actually not a detection of the WR star itself. Thus, the position of 
HD 5980 in Fig. 15 and the classification following from it, should be disregarded. Finally, NGC 346:KWBBe 200 is a curious object, which, from its IRS spectrum appears to be a YSO, but has characteristics in common with $\mathrm{B}[\mathrm{e}]$ stars (See Appendix A). It falls well outside the box defined for YSO, C-PN and C-PAGB, perhaps due to its unusual nature.

\subsubsection{Oxygen-rich AGB stars and RSGs}

Since the main purpose of the classification scheme by Matsuura et al. (2013) is to determine the dust production by evolved stars distinguished by carbon-rich and oxygen-rich chemistry, the subdivision in types of evolved stars within the oxygen-rich class is less important. In fact, in the first step, all types of oxygen-rich evolved stars (AGB stars and RSGs) are lumped together as RSG/O-AGB (Fig. 14), while in the second step a subdivision is made between O-AGB (which also includes RSG) and O-AGB/O-PAGB (Fig. 15). The latter category contains the more evolved O-AGB stars. Thus, a total of three partially overlapping categories exist. The RSG/OAGB category contains objects that are classified as such in the first step, but remained unclassified in the second step. This class contains only three objects (2MASS J00515018-7250496, 2MASS J00524017-7247276 and IRAS 00350-7436), which are all CAGB according to their IRS spectroscopy, and are already discussed in Section 5.2.1.

The 24 objects classified as O-AGB in the second step are indeed all O-AGB or RSG objects according to their IRS spectroscopy. However, the category O-PAGB/O-AGB contains a more diverse range of objects. Of the eight SMC IRS objects classified by the method of Matsuura et al. (2013) to be in this category only four are genuine O-rich evolved stars: IRAS F00483-7347 (SMC IRS 98) is an RSG according to our classification, LHA 115-S 38 (SMC IRS 257) is found to be a O-PAGB object, and 2MASS J00463159-7328464 (SMC IRS 121) and HV 12956 (SMC IRS 277) are O-AGB stars. The other objects in this category include two carbon-rich evolved stars, 2MASS J00444111-7321361 (SMC IRS 268), a C-PAGB object, and Lin 343 (SMC IRS 161), a C-PN. Both of these objects should have fallen in the YSO/C-PN/C-PAGB, but with $K_{S}-[24]$ just below 8 mag they both just miss the cutoff. Since Fig. 15 does not show any O-rich objects in the vicinity of the $\mathrm{K}-[24]=8$ mag cutoff between $\mathrm{C}$-rich and O-rich post-AGB objects, a case can be made to lower the cutoff slightly. Finally, the remaining two misclassified objects in the O-PAGB/O-AGB category by Matsuura et al. (2013) are spectrally classified as B[e] stars in the OTHER category. They are RMC 50 (SMC IRS 193) and Lin 250 (SMC IRS 262).

When checking the reverse direction, we find that all objects classified by us as O-AGB are indeed identified as either O-AGB or as O-PAGB/O-AGB according to the classification scheme by Matsuura et al. (2013). However, this classification scheme misclassifies all objects identified as O-EAGB by us. According to the scheme by Matsuura et al. (2013), they are either C-AGB (See Section 5.2.1; four out of eight objects), or remain unclassified, as they have [3.6]$[8.0]<0.7$ mag, rendering them unclassifiable in Fig. 14, and have $K_{S}-[8.0]<0.5 \mathrm{mag}$ and $K_{S}-[24]<0.5 \mathrm{mag}$, which causes them to fall outside all boundaries in Fig. 15. This also happens to six of the 22 objects classified by us to be RSGs; one is misclassified as a C-AGB (See Section 5.2.1), and the other five do not receive a classification because their colours are too blue (i.e. their mass-loss rates are too low).
Our sample of 209 targets with IRS spectra contains only one oxygen-rich post-AGB star, which agrees with the O-PAGB/O-AGB classification from Matsuura et al. (2013).

\subsection{Sewiło et al. (2013)}

The third classification scheme that we compare with is the method developed by Whitney et al. (2008) and refined by Sewiło et al. (2013) to select YSO candidates. From the set of CMDs used by Whitney et al. (2008), Sewiło et al. (2013) selected a combination of five different CMDs to select YSO candidates in the SMC. Two of these diagrams are reproduced in this work, with the 209 IRS point sources overplotted (Figs 16 and 17). After the initial colour selection of YSO candidates, Sewiło et al. (2013) performed additional tests, including a visual inspection of the imaging, a check against the SIMBAD and other catalogues for known non-YSO sources, and fitting against the YSO SED grid calculated by Robitaille et al. (2006). Sewiło et al. (2013) arrive at a list of approximately 1000 'high-reliability' and 'probable' YSOs in the SMC.

Although Figs 16 and 17 appear to show considerable amount of pollution from non-YSO IRS staring-mode targets, as well as many confirmed YSOs based on the IRS data straying out of the defined boxes, it is the combination of the five CMDs, along with the additional non-colour based checks that yields a highly reliable YSO candidate list. Indeed, the Sewiło et al. (2013) classification favours reliability over completeness, and CMD areas with significant pollution due to other sources (background galaxies, PNe) have been excluded. The list of 209 IRS staring-mode point sources contains nine objects classified as probable YSOs by Sewiło et al. (2013), and 45 high-reliability YSO candidates. Of the high-reliability YSOs for which IRS observations are available, we find that the vast majority are indeed YSOs (covering all four classes). Only two objects turn out to be something different: $\mathrm{H}$ in region IRAS 00436-7321 (SMC IRS 310) and C-PAGB object 2MASS J01054645-7147053 (SMC IRS 243). Among the probable YSO candidates, the success rate is lower: four out of nine are not YSOs, upon inspection of their IRS spectra. RMC 50 (SMC IRS 193) is a B[e] star, while SMP SMC 11 (SMC IRS 32), LHA 115-N 43 (SMC IRS 155) and Lin 49 (SMC IRS 292) are actually C-PN objects.

Furthermore, there are three YSO-3 type objects, as spectrally classified, that are not identified as YSO candidates by Sewiło et al. (2013). These are NGC 346:KWBBe 200 (SMC IRS 19), 2MASS J00465185-7315248 (SMC IRS 269) and LHA 115-N 8 (SMC IRS 274).

\section{SUMMARY}

We have analysed all 311 Spitzer-IRS staring-mode observations within the SAGE-SMC IRAC and MIPS coverage of the SMC (Gordon et al. 2011). After removing IRS observations of extended emission, blank sky and duplicate observations, we find that 209 unique IRAC point sources were targeted. We applied the infrared spectroscopic classification method devised by Woods et al. (2011), with the addition of one more category, namely O-EAGB stars (early-type oxygen-rich AGB stars). We find that the Spitzer-IRS staring-mode sample of point sources in the SMC contains 51 YSOs, subdivided in 14 embedded YSOs (YSO-1), 5 less-embedded YSOs (YSO-2), 22 evolved YSOs (YSO-3) and 10 HAeBe type objects (YSO-4). Furthermore, we find the sample contains 46 oxygen-rich evolved stars: 8 O-EAGB stars, 11 O-AGB stars, 22 RSGs, 1 OPAGB object and 4 O-PN objects. 62 objects turn out to be carbonrich evolved stars, namely 39 C-AGB stars, 3 C-PAGB objects and 


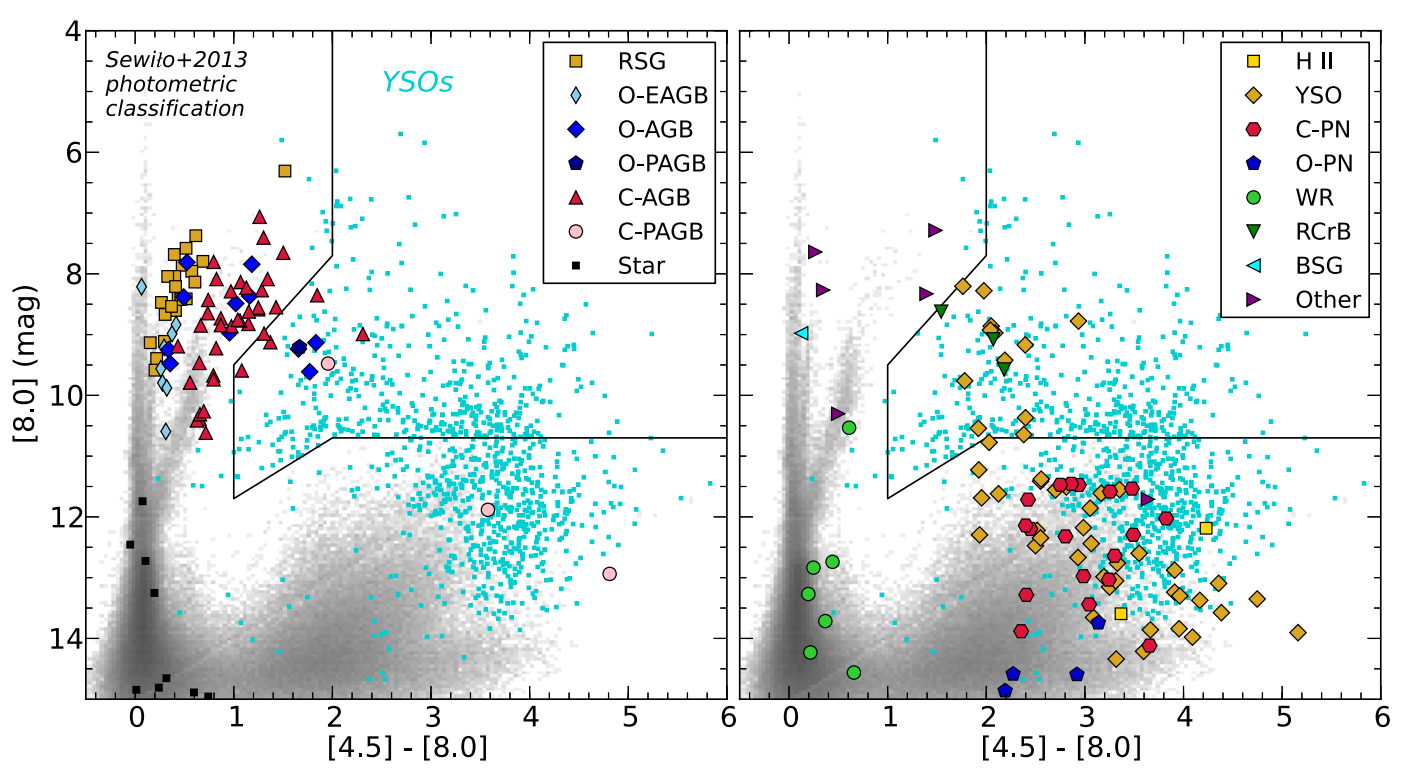

Figure 16. One of the CMD diagrams used in the Sewiło et al. (2013) selection method for candidate YSOs, based on the work by Whitney et al. (2008). This [8.0] versus [4.5]-[8.0] CMD diagram shows the cut used in solid black lines. In grey-scale, the SAGE-SMC PSC is shown in the form of a density plot. The light blue dots represent the YSO candidates finally selected by Sewiło et al. (2013). The coloured symbols show the location of the objects spectrally classified in this work, spread out over two panels for clarity (Sewiło et al. 2013), based on Whitney et al. 2008.

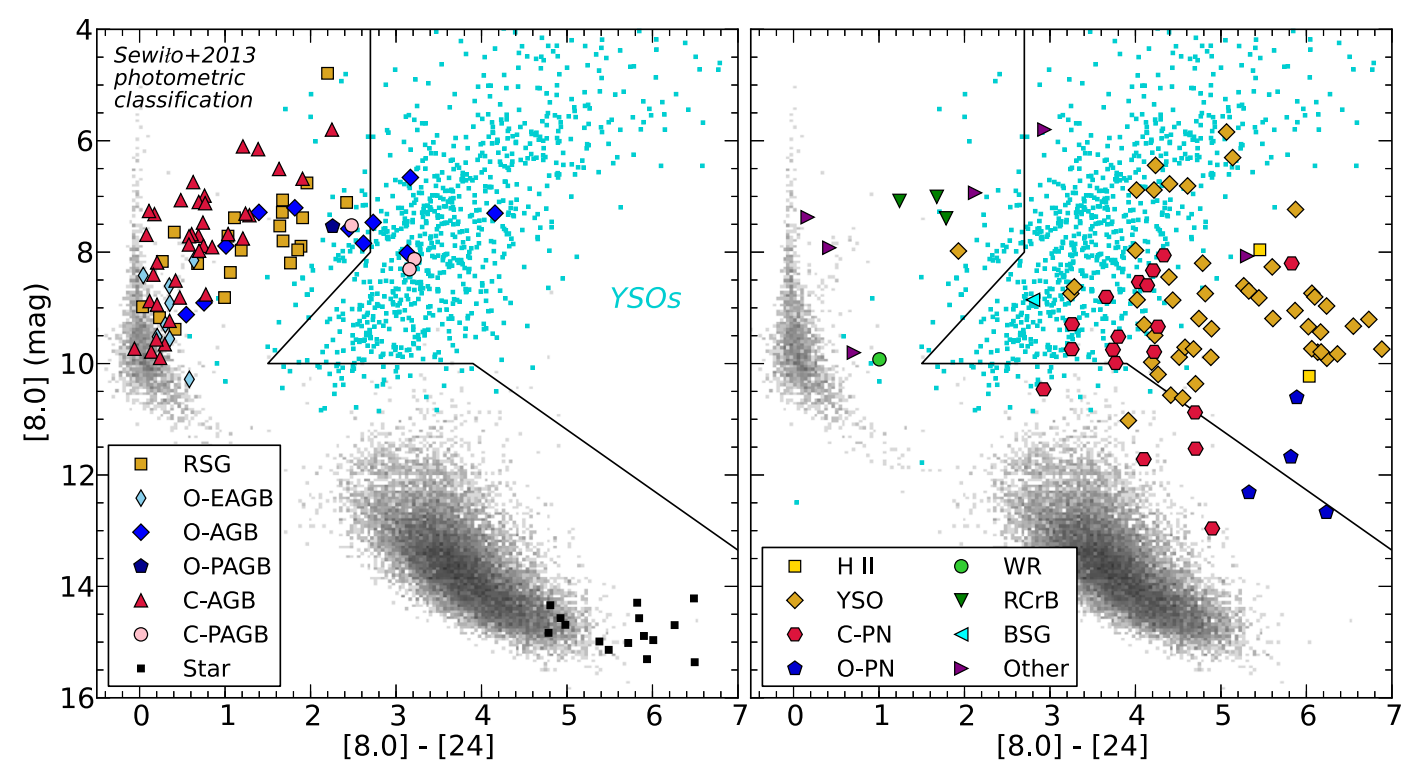

Figure 17. As Fig. 16, but now for [8.0] versus [8.0]-[24].

20 C-PN objects. The sample also includes $3 \mathrm{R}$ CrB stars, 1 BSG and 10 WR stars. 27 objects show stellar photospheres, 23 of which were selected based on their MIPS-[24] excess, and labelled by us as dusty OB stars. It turns out that the $24-\mu \mathrm{m}$ emission is in general not related to the host OB star (Adams et al. 2013; Sheets et al. 2013). Finally, the sample includes a small number of other objects (OTHER), which do not follow from the classification method. These include $2 \mathrm{~B}$ [e] stars, 2 foreground oxygen-rich AGB stars, an $\mathrm{S}$ star, and a symbiotic star.

In this work, we have compared the resulting spectral classifications with the outcome of photometric classification schemes. It should be noted that the spectroscopic observations are obtained in 14 different observing programmes, with a diverse range of science goals. Thus, there is no homogeneous coverage of colour- magnitude space, and it will be impossible to quantify the goodness of any given photometric classification method. Furthermore, the observing programmes tend to target rare types of sources in a disproportionate amount, and some of these rare type of sources ( $R$ CrB stars, WR stars, B [e] stars, etc.) are not included in photometric classification schemes, precisely because they are rare. Thus, these objects tend to pollute the classification schemes discussed here, but statistically they are rather insignificant.

We reviewed three different photometric classification schemes for infrared sources in the SMC: the schemes by Boyer et al. (2011) and Matsuura et al. (2013) for evolved stars, and the classification scheme to select candidate YSOs by Sewiło et al. (2013). The latter scheme is not a pure photometric classification, as it includes additional steps, such as visual inspection of the direct environment of 
the point source in imaging, checks against existing catalogues and fitting against the grid of YSO SEDs calculated by Robitaille et al. (2006). However, as discussed in Section 5.3, the 54 overlapping sources from this work with the resulting YSO candidate list are mostly correctly classified. Only a few sources are misclassified in either direction, i.e. three spectroscopically confirmed YSOs were not on the candidate list by Sewiło et al. (2013), and six sources on the candidate list were found to be something else upon inspection of their IRS spectroscopy. All-in-all, we conclude that the YSO candidate list produced by Sewiło et al. (2013) is reliable, with 48/54 sources indeed being YSOs and the high-reliability sources doing better than the probable sources. Only three spectroscopically confirmed YSOs were missed due to unusual infrared colours.

The two photometric classification methods for evolved stars can be directly compared to each other. The method by Boyer et al. (2011) has its focus on identifying the entire dusty evolved star population, while the Matsuura et al. (2013) method is mainly driven by the motivation to determine the carbon-rich and oxygen-rich dust production rates. Thus, in the later method, correct identification of lower mass-loss rate stars is not so important. Both methods can be used to estimate the integrated dust production rate. Due to the low metallicity of the SMC, carbon-rich evolved stars are more numerous (Blanco, Blanco \& McCarthy 1978, 1980; Lagadec et al. 2007), and the carbon stars have, on average, the highest massloss rates. Thus, identifying carbon stars correctly is important as they dominate the dust budget (Matsuura et al. 2009; Boyer et al. 2012; Riebel et al. 2012). Apart from rare object classes, Boyer et al. (2011) very efficiently separate C-AGB stars from the other classes, only classifying one non-C-AGB star as C-AGB star, while classifying all genuine C-AGB objects as C-AGB. Matsuura et al. (2013) do slightly worse, with three genuine C-AGB objects being classified as something else, and a number of objects, including some rare types, incorrectly classified as C-AGB star.

On the oxygen-rich side, we find that Matsuura et al. (2013) perform better for the high-mass-loss rate objects (O-AGB stars), but that they perform rather poorly on the low-mass rate objects (O-EAGB stars), while the performance of the Boyer et al. (2011) classification method is reversed because of overlap between highmass AGB stars and RSGs near the classical AGB limit.

Finally, we note that of the two steps involved in the classification method by Matsuura et al. (2013), the second step (Fig. 15) matches very well with the actual spectroscopic classification. It has been introduced by Matsuura et al. (2013) as a correction on the first step from Matsuura et al. (2009), but almost all photometric classifications currently included in the online table described by Table 2 correspond to the second step, and most of the time match the spectroscopic classification, rendering the first step practically unnecessary. Thus, in case of the Matsuura et al. (2013) classification scheme, applying only the second step, as demonstrated in Fig. 15, would suffice for dusty sources.

\section{ACKNOWLEDGEMENTS}

The authors wish to thank Paul Ruffle's partner Rose Wheeler. Rose provided access to Paul's notes and files, which allowed us to finish this work. PMER thanks Academia Sinica Institute of Astronomy and Astrophysics (ASIAA) for their financial support and hospitality during the preparation of this work. The authors thank David Whelan for making available Spitzer spectra of point sources described in his 2013 paper. Astrophysics at JBCA is supported by STFC. FK acknowledges support from the former National Science Council and the Ministry of Science and Technology in the form of grants NSC100-2112-M-001-023-MY3 and MOST1032112-M-001-033-. BAS acknowledges support from NASA ADP NNX11AB06G. RSz acknowledges support from the Polish NCN grant 2011/01/B/ST9/02031. The research presented here was conducted within the scope of the HECOLS International Associated Laboratory, supported in part by the Polish NCN grant DEC2013/08/M/ST9/00664 (E.L.; R.Sz). This work is based (in part) on observations made with the Spitzer Space Telescope, obtained from the NASA/IPAC Infrared Science Archive, both of which are operated by the Jet Propulsion Laboratory, California Institute of Technology under a contract with the National Aeronautics and Space Administration. This publication makes use of data products from the Wide-field Infrared Survey Explorer, which is a joint project of the University of California, Los Angeles, and the Jet Propulsion Laboratory/California Institute of Technology, funded by the National Aeronautics and Space Administration. Some of the data presented in this paper were obtained from the Mikulski Archive for Space Telescopes (MAST). STScI is operated by the Association of Universities for Research in Astronomy, Inc., under NASA contract NAS5-26555. Support for MAST for non-Hubble Space Telescope (HST) data is provided by the NASA Office of Space Science via grant NNX13AC07G and by other grants and contracts. This research has also made use of the SAGE CASJobs data base, which is made possible by the Sloan Digital Sky Survey Collaboration; SAOImage DS9, developed by Smithsonian Astrophysical Observatory; the VizieR catalogue access tool, CDS, Strasbourg, France; the SIMBAD data base, operated at CDS, Strasbourg, France; and NASA's Astrophysics Data System Bibliographic Services.

\section{REFERENCES}

Abia C., Cunha K., Cristallo S., de Laverny P., Domínguez I., Recio-Blanco A., Smith V. V., Straniero O., 2011, ApJ, 737, L8

Adams J. J. et al., 2013, ApJ, 771, 112

Allard F., Homeier D., Freytag B., 2011, in Johns-Krull C., Browning M. K., West A. A., eds, ASP Conf. Ser. Vol. 448, 16th Cambridge Workshop on Cool Stars, Stellar Systems, and the Sun. Astron. Soc. Pac., San Francisco, p. 91

Ardeberg A., Maurice E., 1977, A\&AS, 30, 261

Arp B. H., 1959, AJ, 64, 254

Azzopardi M., Breysacher J., 1980, A\&AS, 39, 19

Azzopardi M., Vigneau J., 1982, A\&AS, 50, 291

Balona L. A., 1992, MNRAS, 256, 425

Barba R. H., Niemela V. S., Baume G., Vazquez R. A., 1995, ApJ, 446, L23 Barba R. H. et al., 1996, in Niemela V., Morrell N., Pismis P., TorresPeimbert S., eds, Rev. Mex. Astron. Astrofis. Ser. Conf., 5, 85 Barnbaum C., Kastner J. H., Morris M., Likkel L., 1991, A\&A, 251, 79 Basinski J. M., Bok B. J., Bok P. F., 1967, MNRAS, 137, 55

Battinelli P., 1991, A\&A, 244, 69

Bayne G. et al., 2002, MNRAS, 331, 609

Bernard-Salas J., Peeters E., Sloan G. C., Gutenkunst S., Matsuura M., Tielens A. G. G. M., Zijlstra A. A., Houck J. R., 2009, ApJ, 699, 1541 Bica E. L. D., Schmitt H. R., 1995, ApJS, 101, 41 Blanco B. M., Blanco V. M., McCarthy M. F., 1978, Nature, 271, 638 Blanco V. M., Blanco B. M., McCarthy M. F., 1980, ApJ, 242, 938 Blanco V. M., Frogel J. A., McCarthy M. F., 1981, PASP, 93, 532 Blum R. D. et al., 2006, AJ, 132, 2034

Bolatto A. D. et al., 2007, ApJ, 655, 212

Bonanos A. Z. et al., 2010, AJ, 140, 416

Bouchet P., Lequeux J., Maurice E., Prevot L., Prevot-Burnichon M. L., 1985, A\&A, 149, 330

Boyer M. L. et al., 2011, AJ, 142, 103

Boyer M. L. et al., 2012, ApJ, 748, 40

Brett J. M., 1991, MNRAS, 249, 538

Breysacher J., François P., 2000, A\&A, 361, 231 
Breysacher J., Perrier C., 1980, A\&A, 90, 207

Breysacher J., Moffat A. F. J., Niemela V. S., 1982, ApJ, 257, 116

Buscombe W., Kennedy P. M., 1962, J. R. Astron. Soc. Can., 56, 113

Castilho B. V., Gregorio-Hetem J., Spite F., Spite M., Barbuy B., 1998, A\&AS, 127, 139

Catchpole R. M., Feast M. W., 1981, MNRAS, 197, 385

Cellone S. A. et al., 1996, in Niemela V., Morrell N., Pismis P., TorresPeimbert S., eds, Rev. Mex. Astron. Astrofis. Ser. Conf., 5, 123

Charmandaris V., Heydari-Malayeri M., Chatzopoulos E., 2008, A\&A, 487, 567

Chen Y.-P., Trager S. C., Peletier R. F., Lançon A., Vazdekis A., Prugniel P., Silva D. R., Gonneau A., 2014, A\&A, 565, A117

Chiosi E., Vallenari A., Held E. V., Rizzi L., Moretti A., 2006, A\&A, 452, 179

Cidale L., Zorec J., Tringaniello L., 2001, A\&A, 368, 160

Cioni M.-R. L. et al., 2003, A\&A, 406, 51

Cioni M.-R. L., Girardi L., Marigo P., Habing H. J., 2006, A\&A, 448, 77

Clark J. S., Bartlett E. S., Coe M. J., Dorda R., Haberl F., Lamb J. B., Negueruela I., Udalski A., 2013, A\&A, 560, A10

Cohen J. G., 1984, AJ, 89, 1779

Contursi A. et al., 2000, A\&A, 362, 310

Crowther P. A., Hadfield L. J., 2006, A\&A, 449, 711

Dachs J., 1970, A\&A, 9, 95

de Boer K. S., Savage B. D., 1980, ApJ, 238, 86

De Smedt K., Van Winckel H., Karakas A. I., Siess L., Goriely S., Wood P. R., 2012, A\&A, 541, A67

De Smedt K., Van Winckel H., Kamath D., Karakas A. I., Siess L., Goriely S., Wood P., 2014, A\&A, 563, L5

Dopita M. A., Lawrence C. J., Ford H. C., Webster B. L., 1985, ApJ, 296, 390

Dopita M. A., Bell J. F., Chu Y.-H., Lozinskaya T. A., 1994, ApJS, 93, 455 Dufour R. J., Killen R. M., 1977, ApJ, 211, 68

Dufton P. L., McErlean N. D., Lennon D. J., Ryans R. S. I., 2000, A\&A, 353,311

Eenens P. R. J., Morris P., 1996, in Niemela V., Morrell N., Pismis P., Torres-Peimbert S., eds, Rev. Mex. Astron. Astrofis. Ser. Conf., 5, 120

Elias J. H., Frogel J. A., Humphreys R. M., 1980, ApJ, 242, L13

Elias J. H., Frogel J. A., Humphreys R. M., 1985, ApJS, 57, 91

Epchtein N. et al., 1999, A\&A, 349, 236

Evans C. J., Howarth I. D., 2008, MNRAS, 386, 826

Evans C. J., Howarth I. D., Irwin M. J., Burnley A. W., Harries T. J., 2004a, MNRAS, 353, 601

Evans C. J., Crowther P. A., Fullerton A. W., Hillier D. J., 2004b, ApJ, 610, 1021

Evans C. J., Lennon D. J., Smartt S. J., Trundle C., 2006, A\&A, 456, 623

Faccioli L., Alcock C., Cook K., Prochter G. E., Protopapas P., Syphers D., 2007, AJ, 134, 1963

Fazio G. G. et al., 2004, ApJS, 154, 10

Feast M. W., 1979, MNRAS, 186, 831

Feast M. W., 1986, in Hunger K., Schoenberner D., Kameswara Rao N., eds, Astrophysics and Space Science Library, Vol. 128, IAU Colloq. 87: Hydrogen Deficient Stars and Related Objects. Reidel, Dordrecht, p. 151

Feast M. W., Thackeray A. D., Wesselink A. J., 1960, MNRAS, 121, 337

Fitzpatrick E. L., Savage B. D., 1983, ApJ, 267, 93

Fitzpatrick E. L., Savage B. D., 1985, ApJ, 292, 122

Foellmi C., Moffat A. F. J., Guerrero M. A., 2003, MNRAS, 338, 360

Foellmi C. et al., 2008, Rev. Mex. Astron. Astrofis., 44, 3

Frogel J. A., Persson S. E., Cohen J. G., 1983, ApJS, 53, 713

García-Hernández D. A. et al., 2009, ApJ, 705, L31

García-Hernández D. A. et al., 2011, ApJ, 737, L30

Garmany C. D., Conti P. S., Massey P., 1987, AJ, 93, 1070

Georgiev L., Koenigsberger G., Hillier D. J., Morrell N., Barbá R., Gamen R., 2011, AJ, 142, 191

González R. F., Koenigsberger G., 2014, A\&A, 561, A105

Gordon K. D. et al., 2011, AJ, 142, 102

Gouliermis D. A., Dolphin A. E., Brandner W., Henning T., 2006, ApJS, 166,549
Gouliermis D. A., Bestenlehner J. M., Brandner W., Henning T., 2010, A\&A, 515, A56

Groenewegen M. A. T., 2004, A\&A, 425, 595

Groenewegen M. A. T., Blommaert J. A. D. L., 1998, A\&A, 332, 25

Groenewegen M. A. T., Blommaert J. A. D. L., Cioni M.-R., Okumura K., Habing H. J., Trams N. R., van Loon J. T., 2000, Mem. Soc. Astron. Ital., 71, 639

Groenewegen M. A. T., Sloan G. C., Soszyński I., Petersen E. A., 2009, A\&A, 506, 1277

Guerrero M. A., Chu Y.-H., 2008, ApJS, 177, 216

Gvaramadze V. V., Pflamm-Altenburg J., Kroupa P., 2011, A\&A, 525, A17

Henize K. G., 1956, ApJS, 2, 315

Henize K. G., Westerlund B. E., 1963, ApJ, 137, 747

Hennekemper E., Gouliermis D. A., Henning T., Brandner W., Dolphin A. E., 2008, ApJ, 672, 914

Heydari-Malayeri M., Selier R., 2010, A\&A, 517, A39

Heydari-Malayeri M., Rauw G., Esslinger O., Beuzit J.-L., 1997, A\&A, 322, 554

Hill V., 1997, A\&A, 324, 435

Hill V., Barbuy B., Spite M., 1997, A\&A, 323, 461

Hog E., Kuzmin A., Bastian U., Fabricius C., Kuimov K., Lindegren L., Makarov V. V., Roeser S., 1998, A\&A, 335, L65

Hoopes C. G., Sembach K. R., Howk J. C., Blair W. P., 2001, ApJ, 558, L35

Hoopes C. G., Sembach K. R., Howk J. C., Savage B. D., Fullerton A. W., 2002, ApJ, 569, 233

Houck J. R. et al., 2004, ApJS, 154, 18

Hummel W., Szeifert T., Gässler W., Muschielok B., Seifert W., Appenzeller I., Rupprecht G., 1999, A\&A, 352, L31

Humphreys R. M., 1979, ApJ, 231, 384

Hunter I., Lennon D. J., Dufton P. L., Trundle C., Simón-Díaz S., Smartt S. J., Ryans R. S. I., Evans C. J., 2008, A\&A, 479, 541

Hunter I. et al., 2009, A\&A, 496, 841

Hutchings J. B., 1966, MNRAS, 131, 299

Ita Y. et al., 2010, PASJ, 62, 273

Jacoby G. H., De Marco O., 2002, AJ, 123, 269

Jones O. C. et al., 2012, MNRAS, 427, 3209

Kamath D., Wood P. R., Soszyński I., Lebzelter T., 2010, MNRAS, 408, 522

Kamath D., Wood P. R., Van Winckel H., 2014, MNRAS, 439, 2211

Kato D. et al., 2007, PASJ, 59, 615

Keller S. C., Wood P. R., Bessell M. S., 1999, A\&AS, 134, 489

Kemper F., Waters L. B. F. M., de Koter A., Tielens A. G. G. M., 2001, A\&A, 369, 132

Kemper F. et al., 2010, PASP, 122, 683

Koenigsberger G., 2004, Rev. Mex. Astron. Astrofis., 40, 107

Koenigsberger G., Moreno E., 2008, Rev. Mex. Astron. Astrofis. Ser. Conf., 33,108

Koenigsberger G., Moffat A. F. J., St-Louis N., Auer L. H., Drissen L., Seggewiss W., 1994, ApJ, 436, 301

Koenigsberger G., Guinan E., Auer L., Georgiev L., 1995, ApJ, 452, L107

Koenigsberger G., Shore S., Guinan E., Auer L., 1996, Rev. Mex. Astron. Astrofis. Ser. Conf., 5, 92

Koenigsberger G., Auer L. H., Georgiev L., Guinan E., 1998a, ApJ, 496, 934

Koenigsberger G., Peña M., Schmutz W., Ayala S., 1998b, ApJ, 499, 889

Koenigsberger G., Georgiev L., Barbá R., Tzvetanov Z., Walborn N. R., Niemela V. S., Morrell N., Schulte-Ladbeck R., 2000, ApJ, 542, 428

Koenigsberger G. et al., 2001, AJ, 121, 267

Koenigsberger G., Fullerton A. W., Massa D., Auer L. H., 2006, AJ, 132, 1527

Koenigsberger G., Georgiev L., Hillier D. J., Morrell N., Barbá R., Gamen R., 2010, AJ, 139, 2600

Koenigsberger G., Morrell N., Hillier D. J., Gamen R., Schneider F. R. N., González-Jiménez N., Langer N., Barbá R., 2014, AJ, 148, 62

Köhler K., Borzyszkowski M., Brott I., Langer N., de Koter A., 2012, A\&A, 544, A76

Kraemer K. E., Sloan G. C., Wood P. R., Price S. D., Egan M. P., 2005, ApJ, 631, L147 
Kraemer K. E., Sloan G. C., Bernard-Salas J., Price S. D., Egan M. P., Wood P. R., 2006, ApJ, 652, L25

Kraus M., Borges Fernandes M., de Araújo F. X., 2010, A\&A, 517, A30

Kučinskas A., Vansevičius V., Sauvage M., Tanabé T., 2000, A\&A, 353, L21

Lagadec E. et al., 2007, MNRAS, 376, 1270

Lasker B. M. et al., 2008, AJ, 136, 735

Lebouteiller V., Bernard-Salas J., Brandl B., Whelan D. G., Wu Y., Charmandaris V., Devost D., Houck J. R., 2008, ApJ, 680, 398

Lebouteiller V., Barry D. J., Spoon H. W. W., Bernard-Salas J., Sloan G. C., Houck J. R., Weedman D. W., 2011, ApJS, 196, 8

Lebzelter T., Wood P. R., 2011, A\&A, 529, A137

Leisy P., Dennefeld M., 1996, A\&AS, 116, 95

Lennon D. J., 1997, A\&A, 317, 871

Lequeux J., Maurice E., Prevot-Burnichon M.-L., Prevot L., RoccaVolmerange B., 1982, A\&A, 113, L15

Levesque E. M., Massey P., Olsen K. A. G., Plez B., Meynet G., Maeder A., 2006, ApJ, 645, 1102

Levesque E. M., Massey P., Olsen K. A. G., Plez B., 2007, ApJ, 667, 202

Lindsay E. M., 1961, AJ, 66, 169

Lipman K., Pettini M., 1995, ApJ, 442, 628

Livanou E. et al., 2007, AJ, 133, 2179

Lloyd Evans T., 1971, The Observatory, 91, 118

Lloyd Evans T., 1978, MNRAS, 183, 319

Lloyd Evans T., 1980, MNRAS, 193, 333

Lloyd Evans T., 1983a, South Afr. Astron. Obs. Circ., 7, 96

Lloyd Evans T., 1983b, MNRAS, 204, 961

Lloyd Evans T., 1985, MNRAS, 212, 955

Lloyd Evans T., 1991, MNRAS, 249, 409

Lloyd Evans T., Glass I. S., Catchpole R. M., 1988, MNRAS, 231, 773

Lombaert R., de Vries B. L., de Koter A., Decin L., Min M., Smolders K., Mutschke H., Waters L. B. F. M., 2012, A\&A, 544, L18

Loup C., Zijlstra A. A., Waters L. B. F. M., Groenewegen M. A. T., 1997, A\&AS, 125, 419

McDonald I., van Loon J. T., 2007, A\&A, 476, 1261

McDonald I., van Loon J. T., Decin L., Boyer M. L., Dupree A. K., Evans A., Gehrz R. D., Woodward C. E., 2009, MNRAS, 394, 831

McDonald I., Zijlstra A. A., Boyer M. L., 2012, MNRAS, 427, 343

Marchenko S. V. et al., 2004, MNRAS, 353, 153

Marshall J. R., van Loon J. T., Matsuura M., Wood P. R., Zijlstra A. A., Whitelock P. A., 2004, MNRAS, 355, 1348

Martayan C., Floquet M., Hubert A. M., Gutiérrez-Soto J., Fabregat J., Neiner C., Mekkas M., 2007, A\&A, 472, 577

Martayan C., Baade D., Fabregat J., 2010, A\&A, 509, A11

Martins F., Hillier D. J., Bouret J. C., Depagne E., Foellmi C., Marchenko S., Moffat A. F., 2009, A\&A, 495, 257

Massey P., 2002, ApJS, 141, 81

Massey P., Duffy A. S., 2001, ApJ, 550, 713

Massey P., Olsen K. A. G., 2003, AJ, 126, 2867

Massey P., Parker J. W., Garmany C. D., 1989, AJ, 98, 1305

Massey P., Olsen K. A. G., Parker J. W., 2003, PASP, 115, 1265

Massey P., Levesque E. M., Olsen K. A. G., Plez B., Skiff B. A., 2007, ApJ, 660,301

Massey P., Zangari A. M., Morrell N. I., Puls J., DeGioia-Eastwood K., Bresolin F., Kudritzki R.-P., 2009, ApJ, 692, 618

Mathewson D. S., Ford V. L., 1970, AJ, 75, 778

Matsuura M. et al., 2005, A\&A, 434, 691

Matsuura M. et al., 2009, MNRAS, 396, 918

Matsuura M., Woods P. M., Owen P. J., 2013, MNRAS, 429, 2527

Maurice E. et al., 1987, A\&AS, 67, 423

Maurice E., Bouchet P., Martin N., 1989, A\&AS, 78, 445

Meixner M. et al., 2006, AJ, 132, 2268

Meixner M. et al., 2013, AJ, 146, 62

Meyssonnier N., Azzopardi M., 1993, A\&AS, 102, 451

Moffat A. F. J. et al., 1998, ApJ, 497, 896

Monet D. G. et al., 2003, AJ, 125, 984

Monk D. J., Barlow M. J., Clegg R. E. S., 1988, MNRAS, 234, 583

Morgan D. H., 1984, MNRAS, 208, 633
Morgan D. H., 1995, A\&AS, 112, 445

Morgan D. H., Vassiliadis E., Dopita M. A., 1991, MNRAS, 251, 51p

Morris P. W., Eenens P. R. J., Hanson M. M., Conti P. S., Blum R. D., 1996, ApJ, 470, 597

Nazé Y., Hartwell J. M., Stevens I. R., Corcoran M. F., Chu Y.-H., Koenigsberger G., Moffat A. F. J., Niemela V. S., 2002, ApJ, 580, 225

Nazé Y., Manfroid J., Stevens I. R., Corcoran M. F., Flores A., 2004, ApJ, 608, 208

Neugent K. F., Massey P., Skiff B., Drout M. R., Meynet G., Olsen K. A. G., 2010, ApJ, 719, 1784

Nugis T., Annuk K., Hirv A., 2007, Balt. Astron., 16, 227

Oey M. S., King N. L., Parker J. W., 2004, AJ, 127, 1632

Oksala M. E., Kraus M., Arias M. L., Borges Fernandes M., Cidale L., Muratore M. F., Curé M., 2012, MNRAS, 426, L56

Oksala M. E., Kraus M., Cidale L. S., Muratore M. F., Borges Fernandes M., 2013, A\&A, 558, A17

Oliveira J. M. et al., 2011, MNRAS, 411, L36

Oliveira J. M. et al., 2013, MNRAS, 428, 3001

Osmer P. S., 1973, ApJ, 181, 327

Otsuka M., Kemper F., Hyung S., Sargent B. A., Meixner M., Tajitsu A., Yanagisawa K., 2013, ApJ, 764, 77

Otsuka M., Kemper F., Cami J., Peeters E., Bernard-Salas J., 2014, MNRAS, 437, 2577

Parker J. W. et al., 1998, AJ, 116, 180

Payne-Gaposchkin C., Gaposchkin S., 1966, Smithsonian Contrib. Astrophys., 9, 1

Peeters E., Hony S., Van Kerckhoven C., Tielens A. G. G. M., Allamandola L. J., Hudgins D. M., Bauschlicher C. W., 2002, A\&A, 390, 1089

Pellegrini S., Fabbiano G., 1994, ApJ, 429, 105

Pickering E. C., Fleming W. P., 1901, ApJ, 14, 144

Plez B., Smith V. V., Lambert D. L., 1993, ApJ, 418, 812

Pojmanski G., 2002, Acta Astron., 52, 397

Polsdofer E., Seale J., Sewiło M., Vijh U. P., Meixner M., Marengo M., Terrazas M., 2015, AJ, 149, 78

Prevot L., Martin N., Rebeirot E., Maurice E., Rousseau J., 1983, A\&AS, 53,255

Prinja R. K., 1987, MNRAS, 228, 173

Raimondo G., Cioni M.-R. L., Rejkuba M., Silva D. R., 2005, A\&A, 438, 521

Rebeirot E., Azzopardi M., Westerlund B. E., 1993, A\&AS, 97, 603

Richter P. et al., 1998, A\&A, 338, L9

Riebel et al., 2015, ApJ, preprint (arXiv:1505.00845)

Riebel D., Srinivasan S., Sargent B., Meixner M., 2012, ApJ, 753, 71

Rieke G. H. et al., 2004, ApJS, 154, 25

Robitaille T. P., Whitney B. A., Indebetouw R., Wood K., Denzmore P., 2006, ApJS, 167, 256

Sabbi E. et al., 2007, AJ, 133, 44

Sanduleak N., 1968, AJ, 73, 246

Sanduleak N., 1989, AJ, 98, 825

Sanduleak N., MacConnell D. J., Philip A. G. D., 1978, PASP, 90, 621

Savage B. D., de Boer K. S., 1981, ApJ, 243, 460

Sawyer H. B., 1931, Harv. Coll. Obs. Circ., 366, 1

Schmidt T., 1970, A\&A, 6, 294

Schmidtke P. C., Chobanian J. B., Cowley A. P., 2008, AJ, 135, 1350

Schmutz W., Vacca W. D., 1991, A\&AS, 89, 259

Schwering P. B. W., Israel F. P., 1989, A\&AS, 79, 79

Sebo K. M., Wood P. R., 1994, AJ, 108, 932

Sembach K. R., Savage B. D., 1992, ApJS, 83, 147

Sembach K. R., Danks A. C., Savage B. D., 1993, A\&AS, 100, 107

Sewiło M. et al., 2013, ApJ, 778, 15

Shaw R. A. et al., 2010, ApJ, 717, 562

Sheets H. A., Bolatto A. D., van Loon J. T., Sandstrom K., Simon J. D., Oliveira J. M., Barbá R. H., 2013, ApJ, 771, 111

Shetrone M. D., Keane M. J., 2000, AJ, 119, 840

Shull J. M. et al., 2000, ApJ, 538, L73

Simon J. D. et al., 2007, ApJ, 669, 327

Sloan G. C., Kraemer K. E., Matsuura M., Wood P. R., Price S. D., Egan M. P., 2006, ApJ, 645, 1118 
Sloan G. C., Kraemer K. E., Wood P. R., Zijlstra A. A., Bernard-Salas J., Devost D., Houck J. R., 2008, ApJ, 686, 1056

Sloan G. C. et al., 2010, ApJ, 719, 1274

Sloan G. C. et al., 2011, ApJ, 729, 121

Sloan G. C. et al., 2014, ApJ, 791, 28

Smith Neubig M. M., Bruhweiler F. C., 1997, AJ, 114, 1951

Smith V. V., Lambert D. L., 1989, ApJ, 345, L75

Smith V. V., Lambert D. L., 1990, ApJ, 361, L69

Smith V. V., Plez B., Lambert D. L., Lubowich D. A., 1995, ApJ, 441, 735

Smith V. V., Shetrone M. D., Keane M. J., 1999, ApJ, 516, L73

Songaila A., Blades J. C., Hu E. M., Cowie L. L., 1986, ApJ, 303, 198

Soszyński I. et al., 2011, Acta Astron., 61, 217

Soubiran C., Le Campion J.-F., Cayrel de Strobel G., Caillo A., 2010, A\&A, 515, A111

Stahl O., Wolf B., Leitherer C., de Groot M., 1985, A\&AS, 61, 237

Stanghellini L., Shaw R. A., Balick B., Mutchler M., Blades J. C., Villaver E., 2003a, ApJ, 596, 997

Stanghellini L., Villaver E., Shaw R. A., Mutchler M., 2003b, ApJ, 598, 1000

Stanghellini L., García-Lario P., García-Hernández D. A., Perea-Calderón J. V., Davies J. E., Manchado A., Villaver E., Shaw R. A., 2007, ApJ, 671,1669

Stanghellini L., Lee T.-H., Shaw R. A., Balick B., Villaver E., 2009, ApJ, 702,733

Sterken C., Breysacher J., 1997, A\&A, 328, 269

Sterken C., de Groot M., van Genderen A. M., 1998, A\&A, 333, 565

Sturm R. et al., 2013, A\&A, 558, A3

Sylvester R. J., Skinner C. J., Barlow M. J., 1998, MNRAS, 301, 1083

Szeifert T., Stahl O., Wolf B., Zickgraf F.-J., Bouchet P., Klare G., 1993, A\&A, 280, 508

Tanabé T. et al., 1997, Nature, 385, 509

Tanabé T. et al., 2013, PASJ, 65, 55

Thompson G. I., Nandy K., Morgan D. H., Houziaux L., 1988, MNRAS, 230,429

Tisserand P. et al., 2004, A\&A, 424, 245

Tisserand P. et al., 2009, A\&A, 501, 985

Tsalmantza P., Kontizas E., Cambrésy L., Genova F., Dapergolas A., Kontizas M., 2006, A\&A, 447, 89

van Genderen A. M., van Leeuwen F., Brand J., 1982, A\&AS, 47, 591

van Loon J. T. et al., 1998, A\&A, 329, 169

van Loon J. T., Zijlstra A. A., Bujarrabal V., Nyman L.-A., 2001, A\&A, 368, 950

van Loon J. T., Marshall J. R., Zijlstra A. A., 2005, A\&A, 442, 597

van Loon J. T., Cohen M., Oliveira J. M., Matsuura M., McDonald I., Sloan G. C., Wood P. R., Zijlstra A. A., 2008, A\&A, 487, 1055

van Loon J. T., Oliveira J. M., Gordon K. D., Sloan G. C., Engelbracht C. W., 2010, AJ, 139, 1553

Vassiliadis E. et al., 1996, ApJS, 105, 375

Vassiliadis E. et al., 1998, ApJS, 114, 237

Velázquez P. F., Koenigsberger G., Raga A. C., 2003, ApJ, 584, 284

Villar-Sbaffi A., Moffat A. F. J., St-Louis N., 2003, ApJ, 590, 483

Villaver E., Stanghellini L., Shaw R. A., 2004, ApJ, 614, 716

Volk K. et al., 2011, ApJ, 735, 127

Werner M. W. et al., 2004, ApJS, 154, 1

Whelan D. G., Lebouteiller V., Galliano F., Peeters E., Bernard-Salas J. Johnson K. E., Indebetouw R., Brandl B. R., 2013, ApJ, 771, 16

Whitelock P. A., Feast M. W., Menzies J. W., Catchpole R. M., 1989 , MNRAS, 238, 769

Whitney B. A. et al., 2008, AJ, 136, 18

Wilke K., Stickel M., Haas M., Herbstmeier U., Klaas U., Lemke D., 2003, A\&A, 401, 873

Wisniewski J. P., Bjorkman K. S., Bjorkman J. E., Clampin M., 2007a, ApJ, 670,1331

Wisniewski J. P., Bjorkman K. S., Magalhães A. M., Bjorkman J. E., Meade M. R., Pereyra A., 2007b, ApJ, 671, 2040

Wood P. R., Bessell M. S., Fox M. W., 1983, ApJ, 272, 99

Wood P. R., Whiteoak J. B., Hughes S. M. G., Bessell M. S., Gardner F. F., Hyland A. R., 1992, ApJ, 397, 552
Woods P. M. et al., 2011, MNRAS, 411, 1597

Wyrzykowski L. et al., 2004, Acta Astron., 54, 1

Yang M., Jiang B. W., 2012, ApJ, 754, 35

Zacharias N., Urban S. E., Zacharias M. I., Wycoff G. L., Hall D. M., Monet D. G., Rafferty T. J., 2004, AJ, 127, 3043

Zaritsky D., Harris J., Thompson I. B., Grebel E. K., Massey P., 2002, AJ, 123,855

Zhang K., Jiang B. W., Li A., 2009, ApJ, 702, 680

Zickgraf F., 2000, in Smith M. A., Henrichs H. F., Fabregat J., eds, ASP Conf. Ser. Vol. 214, IAU Colloq. 175: The Be Phenomenon in Early-Type Stars. Astron. Soc. Pac., San Francisco, p. 26

Zickgraf F.-J., Wolf B., Leitherer C., Appenzeller I., Stahl O., 1986, A\&A, 163,119

Zijlstra A. A., Loup C., Waters L. B. F. M., Whitelock P. A., van Loon J. T., Guglielmo F., 1996, MNRAS, 279, 32

\section{SUPPORTING INFORMATION}

Additional Supporting Information may be found in the online version of this article:

Table 1. IRS staring-mode targets in the SMC. (http://mnras.oxfordjournals.org/lookup/suppl/doi:10.1093/ mnras/stv1106/-/DC1).

Online table described by Table 2. Classification of point sources targeted in IRS staring mode.

Please note: Oxford University Press (OUP) is not responsible for the content or functionality of any supporting materials supplied by the authors. Any queries (other than missing material) should be directed to the corresponding author for the article.

\section{APPENDIX A: LITERATURE-BASED CLASSIFICATION SUPPORT FOR SAGE-SPEC OBJECTS}

NGC 346 MPG 320 (SMC IRS 16) derives its name from the catalogue by Massey, Parker \& Garmany (1989). Simon et al. (2007) list it as entry no. 79, a probable YSO, consistent with our classification as a YSO-2. However, Lebouteiller et al. (2008) suggest that this point source is not stellar but rather a photon-dominated region (PDR). Whelan et al. (2013), who list this as PS12, follow that suggestion. Furthermore, Kamath, Wood \& Van Winckel (2014) list it as a PN candidate. This source is also known to be an infrared variable (Polsdofer et al. 2015).

NGC 346 MPG 454 (SMC IRS 18) derives its name from the catalogue by Massey et al. (1989). It is located at the position of N66, an H II region (Henize 1956). This source also occurs in the list of candidate PNe by Kamath et al. (2014). Chandra X-ray observations reveal that NGC 346 MPG 454 is one of the brightest blue stars in the NGC 346 cluster (Nazé et al. 2002). Indeed, the infrared part of the spectrum is dominated by a massive YSO (Sabbi et al. 2007; Whelan et al. 2013), in the latter work catalogued as PS9.

NGC 346:KWBBe 200 (SMC IRS 19) is also known as NGC 346 MPG 466 (Massey et al. 1989). It is known to show $\mathrm{H} \alpha$ emission (Meyssonnier \& Azzopardi 1993). It occurs in the list of PN candidates by Kamath et al. (2014). The source was recognized as a classical Be star by Keller, Wood \& Bessell (1999), and it also derives its name from this catalogue. Wisniewski et al. (2007b) performed a study of classical Be stars in NGC 346, and in a detailed follow-up paper on NGC 346:KWBBe 200 alone they concluded that this object is in fact a dusty $\mathrm{B}[\mathrm{e}]$ supergiant (Wisniewski et al. 2007a). However, recently Whelan et al. (2013) suggested that NGC 
346:KWBBe 200 is not an $\mathrm{B}$ [e] supergiant, but rather a Herbig AeBe star, based on the presence of silicate emission features, PAHs and cold dust. In this paper, we classify this object as an YSO-3.

NGC 346 MPG 534 (SMC IRS 20) derives its name from the catalogue by Massey et al. (1989). The location coincides with that of the N66A H II region (Henize 1956), but also with two class I protostars, of which the heaviest is thought to be $16.6 \mathrm{M}_{\odot}$ (Simon et al. 2007), later updated to $17.8 \mathrm{M}_{\odot}$ (Whelan et al. 2013). HeydariMalayeri \& Selier (2010) report that an O8 star is responsible for ionizing the N66A H II region. This source is a known infrared variable (Polsdofer et al. 2015; Riebel et al. 2015).

NGC 346 MPG 605 (SMC IRS 21) was identified for the first time as an $\mathrm{H} \alpha$ emitter (N66B; Henize 1956), and then in the survey of NGC 346 by Massey et al. (1989, star number 605) and classified as a red star. Kamath et al. (2014) list this object as a PN candidate. The star (also known as NGC 346:KWBBe 448) has been then observed by Keller et al. (1999) and classified as having Be spectral type. The spectral type has been established also by Martayan, Baade \& Fabregat (2010) as being B0. The star (95) has also been observed with $F 555 W(\mathrm{~V})$ and $F 814 W$ (I) $H S T$ Advanced Camera for Surveys (ACS) filters by Gouliermis et al. (2006, see also Hennekemper et al. 2008). The star seems to be located close to the border between upper main sequence (UMS) and RGB. Similar observations have been performed by Sabbi et al. (2007, source 17) and the obtained magnitudes may suggest that the star is a RGB source. In the infrared this source has been detected by Infrared Space Observatory (ISO)-CAM (source F - Contursi et al. 2000) and then by Spitzer (see Bolatto et al. 2007). Using SED fits Simon et al. (2007) classify the source as Class I YSO. The SL Spitzer spectrum has been analysed by Whelan et al. (2013, source PS6), and shows silicate emission features, which is rare among young star clusters. The reduced spectrum presented by Whelan et al. (2013) does not show $\mathrm{H}_{2} \mathrm{~S}(3)$ emission at $9.67 \mu \mathrm{m}$, while it seems to be present in our reduced spectrum. Whelan et al. (2013) assume that the optical star related to this IR source has spectral type O5.5V (star number 37 in Hennekemper et al. 2008). However this star is located more than 3 arcsec (about 1 pc) from the IR source, and probably unrelated.

NGC 346 MPG 641 (SMC IRS 22). The optical counterpart, located at about 0.8 arcsec from the extracted position, was detected for the first time by Massey et al. (1989). Better photometry has been obtained by Heydari-Malayeri \& Selier (2010) using the NTT (star N66A-2). The star has been classified as an UMS star (Gouliermis et al. 2006). Near-infrared observations with the VLT have been performed by Gouliermis et al. (2010, ID number 1043), who conclude that this is a classical Be star with approximately B0.5 V spectral type. In the infrared, this source has been detected by ISO-CAM (source H - Contursi et al. 2000), Spitzer (Bolatto et al. 2007), and Herschel (Meixner et al. 2013). The SL Spitzer spectrum has been analysed by Whelan et al. (2013, source PS4). It shows that at this position the extended emission is significant and 'optimal extraction' is required. Using SED fits Simon et al. (2007) classify the source as a Class I YSO, while we conclude that this is an $\mathrm{H} \mathrm{II}$ region.

LHA 115-N 1 (SMC IRS 26) has been detected as an $\mathrm{H} \alpha$ emissionline object by Henize (Henize 1956), and classified by Lindsay (1961) as a probable PN. Its nature as a PN has been confirmed by Sanduleak, MacConnell \& Philip (1978, SMP SMC 1). BernardSalas et al. (2009) already presented the IRS spectrum, showing that this object has an extremely strong $11.3-\mu \mathrm{m}$ feature due to $\mathrm{SiC}$ emission. Further discussion of this object is given by Otsuka et al. (2013).
LHA 115-N 4 (SMC IRS 27) is also catalogued as Lin 16, and SMP SMC 3. It is a well-known SMC PN first catalogued by Henize (1956) as an emission line object. It was subsequently identified as a PN in Lindsay (1961). It was noted as a lower excitation object in Morgan (1984). Various optical spectroscopic observations were carried out in the late 1980s and early 1990s, but the first determination of the C/O ratio was carried out by Vassiliadis et al. (1996) showing that the nebula is carbon-rich. There were no mid-infrared observations of this object prior to the Spitzer spectroscopy which was presented in Bernard-Salas et al. (2009). Their classification of the spectrum as being carbon-rich agrees with ours.

LHA 115-N 6 (SMC IRS 28) is another well-studied PN in the SMC: it is also known as SMP SMC 6, Lin 33, IRAS 00395-7403, LI-SMC 12, GSC 09141-007041, and 2MASS J00412777-7347063. The object was first noted as an emission-line object by Henize (1956) and classified as a PN by Lindsay (1961). The object is bright enough to have been detected by the Infrared Astronomical Satellite (IRAS), and aside from the original IRAS PSC there are pointed observations for this object (Schwering \& Israel 1989). The star was found to be of WR type by E. J. Wampler (unpublished, 1978: reported by Monk, Barlow \& Clegg 1988). The nebula was initially found to be oxygen-rich by Vassiliadis et al. (1996); however, this was revised to carbon-rich by Stanghellini et al. (2009) wherein the carbon abundance is determined from HST observations and combined with a previous ground-based oxygen abundance. The latter result is consistent with our spectral classification of C-PN, which is the same as the classification from Bernard-Salas et al. (2009) where the Spitzer spectrum was first published.

LHA 115-N 67 (SMC IRS 29) was discovered by Henize (1956) and identified as a PN by Lindsay (1961). The object has many other names including Lin 333, SMP SMC 22, and DENIS-P J005837.0-7131548. It also appears to be a supersoft X-ray source detected by Einstein, ROSAT, and XMM (i.e. Pellegrini \& Fabbiano 1994; Sturm et al. 2013). Our classification of the object as an O-rich PN based on the infrared spectrum is consistent with the abundance determinations of Leisy \& Dennefeld (1996) which suggests C/O of 0.275 from combined optical and IUE spectroscopy. Similar abundance results were obtained by Vassiliadis et al. (1998).

Lin 536 (SMC IRS 30) is a PN with cold dust compared to other Magellanic Cloud PNe (SED peaks around $30 \mu \mathrm{m}$ Bernard-Salas et al. 2009). HST ACS prism spectra show it is somewhat carbonpoor, with an $A(C) \sim 7$, while other PNe in the SMC have $A(C)>8$ (Smith et al. 1995).

LHA 115-N 70 (SMC IRS 31) was discovered by Henize (1956), and identified as a PN by Lindsay (1961) under the name Lin 347. It is also known as 2MASS J00591608-7201598 and SMP SMC 24. No carbon abundance was available for this nebula before the measurement of Stanghellini et al. (2009) which, along with a previous oxygen abundance determination from Leisy \& Dennefeld (1996), suggests that the object has C/O just larger than unity. The mid-infrared spectrum of the object was considered as being unusual (but carbon-rich) in Bernard-Salas et al. (2009), and some of the emission features were identified as due to fullerenes by García-Hernández et al. (2011). These are all consistent with our classification as a C-PN.

SMP SMC 11 (SMC IRS 32) is a well-known PN. Other names for this object include LHA 115-N 29, Lin 115, and IRAS 00467-7314. The object was first discovered by Henize (1956) as an emissionline object, and it was classified as a PNe by Lindsay (1961). The carbon-rich nature of the nebula was confirmed from HST observations well before there was any mid-infrared spectroscopic 
information available (i.e. Stanghellini et al. 2003b). Due to the relatively strong mid-infrared emission the object was detected by IRAS in the PSC and was the subject of pointed observations as reported by Schwering \& Israel (1989).

HV 1366 (SMC IRS 36). This O-EAGB object has long been known to be an oxygen-rich AGB star (Catchpole \& Feast 1981), with a period of 293 d (Wood, Bessell \& Fox 1983); it is also known to vary in the infrared (Polsdofer et al. 2015; Riebel et al. 2015). Repeated searches do not show detectable enhanced lithium abundances due to HBB in 4-8 $\mathrm{M}_{\odot}$ AGB stars (Smith \& Lambert 1990; Smith et al. 1995). Sloan et al. (2008) report a revised period of 305 d based on MACHO data, and classify the Spitzer IRS spectrum as 1.N. The corresponding blackbody temperature was found to be $(2270 \pm 560)$ K. OGLE variability data also exist (Soszyński et al. 2011). Boyer et al. (2012) estimate that HV 1366 has a dust massloss rate of $10^{-10.1} \mathrm{M}_{\odot} \mathrm{yr}^{-1}$.

HV 11303 (SMC IRS 38). This object is recognized as a variable oxygen-rich AGB star with a period of $534 \mathrm{~d}$ by Wood et al. (1983), in agreement with our classification of O-EAGB. The star shows enhanced lithium abundances due to hot bottom convective envelope (HBCE) burning (Smith \& Lambert 1990; Smith et al. 1995). The red spectra are dominated by $\mathrm{CN}$ bands, showing signs of enhancements in ${ }^{13} \mathrm{CN}$ (Brett 1991), which is further evidence for hot bottom burning processes. Sloan et al. (2008) assign a spectral classification of $1 . \mathrm{N}$, and fit a blackbody of $2130 \pm 240 \mathrm{~K}$ to the SED.

HV 838 (SMC IRS 39). This object was initially thought to be a RSG, showing strong hydrogen emission (Lloyd Evans 1971; Humphreys 1979), but later recognized to be an oxygen-rich AGB star (Wood et al. 1983). Yang \& Jiang (2012) consider HV 838 as an RSG candidate, but notice that it is indeed an outlier with respect to the RSG population. A period of $654 \mathrm{~d}$ was reported by Lloyd Evans (1985), which was later revised to $629 \mathrm{~d}$ based on the MACHO data (Cioni et al. 2003), however Sloan et al. (2008) report a period of $622 \mathrm{~d}$, based on the MACHO data. Riebel et al. (2015) also find it to vary in the infrared. HV 838 shows Li I 6707- $\AA$ absorption, a consequence of HBCE burning (Smith et al. 1995). The IRS spectrum is classified as $1 . \mathrm{N}$ by Sloan et al. (2008), with a blackbody temperature of $2320 \pm 320 \mathrm{~K}$. García-Hernández et al. (2009) detect $\mathrm{Zr}$ and derive its abundance, and report an upper limit for the $\mathrm{Rb}$ abundance.

HV 11366 (SMC IRS 41). This is an oxygen-rich AGB star with a period of 366 d (Catchpole \& Feast 1981; Wood et al. 1983), in agreement with our classification of O-EAGB. Its spectrum shows strong Li I absorption features at $6707 \AA$, indicative of HBB (Smith \& Lambert 1989, 1990; Plez, Smith \& Lambert 1993; Smith et al. 1995). TiO bands are also visible in the red spectra, but there is no clear sign of $\mathrm{CN}$, and the $\mathrm{C} / \mathrm{O}$ ratio is estimated to be $0.4-0.8$ (Brett 1991). This member of NGC 292 has an effective temperature of $3450 \mathrm{~K}$, with $\log g=0.00 \mathrm{~cm} \mathrm{~s}^{-2}$ and a metallicity of $[\mathrm{Fe} / \mathrm{H}]=-0.42$ (Soubiran et al. 2010). Sloan et al. (2008) classify the Spitzer IRS spectrum as 1.N:O::, with a blackbody temperature of $2230 \pm 300 \mathrm{~K}$. OGLE (Soszyński et al. 2011) and MACHO (Yang \& Jiang 2012) variability data are available.

OGLE SMC-LPV-7488 (SMC IRS 44) is a Mira variable with a period of $434 \mathrm{~d}$, and an amplitude of $1.54 \mathrm{mag}$, derived from its OGLE light curve (Soszyński et al. 2011). It is also variable in the infrared (Polsdofer et al. 2015; Riebel et al. 2015). It is known to be an carbon-rich object from the optical spectrum (Lloyd Evans 1980), consistent with our classification of C-AGB.

HV 12149 (SMC IRS 45). In agreement with our result, this object was recognized as an O-rich AGB star (Wood et al. 1983), with a period of 742 d (Payne-Gaposchkin \& Gaposchkin 1966). This star is also an infrared variable (Polsdofer et al. 2015; Riebel et al. 2015). Enhanced Li I absorption indicative of HBB is not detected (Smith \& Lambert 1990; Smith et al. 1995), however, very strong TiO bands are visible in the red spectrum (Brett 1991), pointing to a low C/O ratio. VO bands are also present (Brett 1991). A search for $\mathrm{OH}$ maser emission only yielded an upper limit of $0.04 \mathrm{Jy}$ at $1612 \mathrm{MHz}$ (Wood et al. 1992). Sloan et al. (2008) classify the Spitzer IRS spectrum as 2.SE8, and the dust mass-loss rate has been estimated to be $10^{-9.0} \mathrm{M}_{\odot} \mathrm{yr}^{-1}$ (Boyer et al. 2012). OGLE (Soszyński et al. 2011) and MACHO (Yang \& Jiang 2012) variability data are available.

HV 11223 (SMC IRS 46) is known to be a semiregular variable from its OGLE light curve, and is also known as OGLE SMCLPV-1141 (Soszyński et al. 2011). Smith et al. (1995) show that it is in fact an O-rich AGB star with Li I 6707- $\AA$ detection, with $\log _{\epsilon}(\mathrm{Li})=3.5$.

Dachs SMC 2-37 (SMC IRS 47). This object, also known as Massey SMC 59803, is known to be an M supergiant (Humphreys 1979), in agreement with our classification. Its spectral type is reported to be M0-1 I (Massey \& Olsen 2003), although Levesque et al. (2006) believe it to be K2-3 I. Modelling of optical spectra results in determinations of $T_{\text {eff }}=4100 \mathrm{~K}, A_{V}=0.93, \log$ $g=0.0$ (model) or -0.2 (actual) and $R=900 \mathrm{R}_{\odot}$ (Levesque et al. 2006). The Spitzer IRS spectrum is classified as 2.SE4u (Sloan et al. 2008), looking similar to the ISO-SWS spectra of RSGs in the $\mathrm{h}$ and $\chi$ Perseus supergiant cluster (Sylvester, Skinner \& Barlow 1998), and a C class PAH spectrum (Peeters et al. 2002). The period is derived from MACHO data and found to be $547 \pm 17 \mathrm{~d}$, with a long secondary period of $2543 \pm 38$ d (Yang \& Jiang 2012). This source is also variable in the infrared (Polsdofer et al. 2015).

SMC-WR9 (SMC IRS 57) is a WN3ha WR star with a possible spectroscopic binary orbit of 37.6 d (Morgan, Vassiliadis \& Dopita 1991; Massey \& Duffy 2001; Foellmi, Moffat \& Guerrero 2003). The star is modelled to be $94000-168000 \mathrm{~L}_{\odot}$ and to have a wind velocity of $1616 \mathrm{~km} \mathrm{~s}^{-1}$ and a mass-loss rate $\dot{M}$ of 1.4 $3.5 \times 10^{-6} \mathrm{M}_{\odot} \mathrm{yr}^{-1}$ (Nugis, Annuk \& Hirv 2007).

SMC-WR10 (SMC IRS 58), also known as 2MASS J00452890-7304458, is a single WN3ha WR star, lying in nebulous knot e12 within NGC 249 (Massey \& Duffy 2001; Foellmi et al. 2003; Crowther \& Hadfield 2006). The star is modelled to be $120000-240000 \mathrm{~L}_{\odot}$ and to have a wind velocity of $1731 \mathrm{~km}$ $\mathrm{s}^{-1}$ and $\dot{M}=2-5 \times 10^{-6} \mathrm{M}_{\odot} \mathrm{yr}^{-1}$ (Nugis et al. 2007). Its spectrum is noted for having strong nebulosity (Massey \& Duffy 2001).

SMC-WR12 (SMC IRS 60), also known as Massey SMC 54730 (Massey 2002), was identified as a WN3-4.5 WR star by Massey, Olsen \& Parker (2003).

GSC 09141-05631(SMC IRS 61), also known as SMC-WR1, is a WR star of spectral type WN3ha (Bonanos et al. 2010).

SMC-WR2 (SMC IRS 64) is a single WN5ha WR star with surrounding nebulosity (Azzopardi \& Breysacher 1980; Massey \& Duffy 2001; Foellmi et al. 2003). The star has an estimated luminosity of $320000 \mathrm{~L}_{\odot}$ and $\dot{M}=7-8 \times 10^{-6} \mathrm{M}_{\odot} \mathrm{yr}^{-1}$ (Martins et al. 2009).

SMC-WR3 (SMC IRS 66) is a binary (WN3h+O9:) WR star with a spectroscopic period of 10 d (Azzopardi \& Breysacher 1980; Massey \& Duffy 2001; Foellmi et al. 2003). It is thought to be a lower mass, evolved, H-rich object evolved from a $40-50-\mathrm{M}_{\odot}$ star (Marchenko et al. 2004). This star appears to be interacting with the surrounding ISM, producing a kidney-shaped nebulosity (Gvaramadze, Pflamm-Altenburg \& Kroupa 2011). 
SMC-WR4 (SMC IRS 68) is a binary WN6h+ WR star with a photometric period of 6.55 d (Azzopardi \& Breysacher 1980; Massey \& Duffy 2001; Foellmi et al. 2003). The system has an estimated luminosity of $800000 \mathrm{~L}_{\odot}$ and a combined effective temperature of $\sim 42500 \mathrm{~K}$ (Martins et al. 2009).

RMC 31 (SMC IRS 70), also known as SMC-WR6, is a 6.5-d binary (WN4:+O6.5I:) WR star showing X-ray emission and longperiod variability (Azzopardi \& Breysacher 1980; Massey \& Duffy 2001; Foellmi et al. 2003).

SMC-WR11 (SMC IRS 85), also known as 2MASS J00520738-7235385, is a single WN4h:a WR star with appreciable reddening (Massey \& Duffy 2001; Foellmi et al. 2003). The star is modelled to be $210000-390000 \mathrm{~L}_{\odot}$ and have a wind velocity of $1616 \mathrm{~km} \mathrm{~s}^{-1}$ and $\dot{M}=2-5 \times 10^{-6} \mathrm{M}_{\odot} \mathrm{yr}^{-1}$ (Nugis et al. 2007).

2MASS J00461632-7411135 (SMC IRS 94), also known as MSX SMC 014, was classified as an R CrB candidate by Kraemer et al. (2005), based on the IRS spectrum and near-IR variability. This classification is supported by its 3-4- $\mu \mathrm{m}$ spectrum from van Loon et al. (2008).

2MASS J00364631-7331351 (SMC IRS 95) is also known as MSX SMC 029. PAH features in the IRS spectrum of this source (Kraemer et al. 2006) and the spectral appearance of the 3-4- $\mu \mathrm{m}$ wavelength range (van Loon et al. 2008) point to a C-rich post-AGB star.

2MASS J00455394-7323411 (SMC IRS 96) is also known as MSX SMC 036. Spectra from Groenewegen et al. (2009), van Loon et al. (2008), and Sloan et al. (2006) all reveal the presence of $\mathrm{C}_{2} \mathrm{H}_{2}$, demonstrating that this a C-rich AGB star. Sloan et al. (2006) also note $\mathrm{SiC}$ and $\mathrm{MgS}$ dust features in the IRS spectrum. OGLEIII (Soszyński et al. 2011) measure a period of $553 \mathrm{~d}$, and an Iband amplitude of $2.01 \mathrm{mag}$. It is also variable in the infrared (Polsdofer et al. 2015). Groenewegen et al. (2009) find $\dot{M}=3.6 \times$ $10^{-6} \mathrm{M}_{\odot} \mathrm{yr}^{-1}$.

2MASS J00430590-7321406 (SMC IRS 97), also known as MSX SMC 054, is a C-rich AGB star, as demonstrated by spectra from Groenewegen et al. (2009), van Loon et al. (2008), and Sloan et al. (2006), which all confirm the presence of $\mathrm{C}_{2} \mathrm{H}_{2}$. Sloan et al. (2006) also note $\mathrm{SiC}$ and $\mathrm{MgS}$ dust features in the IRS spectrum. Groenewegen et al. (2009) find $\dot{M}=3.9 \times 10^{-6} \mathrm{M}_{\odot} \mathrm{yr}^{-1}$. Its period is not known, although it is an infrared variable (Polsdofer et al. 2015).

IRAS F00483-7347 (SMC IRS 98) is also known as S9 and MSX SMC 055. IR spectra from Groenewegen et al. (2009) and van Loon et al. (2008) indicate an M star, with spectral type M8. Optical spectra show $\mathrm{ZrO}+\mathrm{H}_{2} \mathrm{O}$ and $\mathrm{TiO}$ absorption (Groenewegen \& Blommaert 1998). Variability information $\left(P=1749 \mathrm{~d}, A m p_{I}=0.87 \mathrm{mag}\right.$; Groenewegen et al. 2009), a Li-overabundance (Castilho et al. 1998), and Rb enhancement (García-Hernández et al. 2009) also point to an AGB star. Groenewegen et al. (2009) classify the star as a Mira, and a candidate super-AGB $\operatorname{star}\left(\dot{M}=5.3 \times 10^{-6} \mathrm{M}_{\odot} \mathrm{yr}^{-1}\right)$. No maser emission has been detected (van Loon et al. 2001; Marshall et al. 2004). We classify it as an RSG by virtue of its $M_{\text {bol }}$ $=-7.3$ mag. It is also in the Riebel et al. (2015) list of infrared variables.

IRAS F00448-7332 (SMC IRS 99), also known as S6 or MSX SMC 060 is known to be a carbon-rich AGB star. Spectra from Groenewegen et al. (2009), van Loon et al. (2008), and Sloan et al. (2006) all confirm the presence of $\mathrm{C}_{2} \mathrm{H}_{2}$. Sloan et al. (2006) also note $\mathrm{SiC}$ and $\mathrm{MgS}$ dust features in the IRS spectrum. Groenewegen et al. (2009) measure a period of 429 d, a $K$-band amplitude of $0.55 \mathrm{mag}$, and $\dot{M}=8.9 \times 10^{-6} \mathrm{M}_{\odot} \mathrm{yr}^{-1}$. It is also variable in the infrared (Polsdofer et al. 2015; Riebel et al. 2015). Sloan et al.
(2006) note that its pulsation properties and photometry make it look similar to $\mathrm{R} \mathrm{CrB}$ stars, but its spectrum does not support that classification. We classify it as an C-AGB star, consistent with the literature.

2MASS J00485250-7308568 (SMC IRS 100), also known as MSX SMC 066, was suggested to be a carbon star by Raimondo et al. (2005) based on its near-IR colours, consistent with our classification and that of Sloan et al. (2006) as a carbon-rich AGB star. Although listed as a Mira by Soszyński et al. (2011), there is some question regarding its primary period, given as $519.5 \mathrm{~d}$ therein, but as $267 \mathrm{~d}$ by Groenewegen (2004) with a secondary period of 512 d. It is also in the Riebel et al. (2015) list of infrared variables.

2MASS J00592336-7356010 (SMC IRS 101), also known as MSX SMC 093, was identified as a carbon-rich object by Sloan et al. (2006), who noted it has a particularly weak SiC emission feature. Soszyński et al. (2011) used the OGLE light curve and near-infrared photometry to classify it as a carbon-rich semiregular variable with a primary period of $457 \mathrm{~d}$. We classify it here as C-AGB.

2MASS J00450214-7252243 (SMC IRS 102), also known as MSX SMC 105, was identified as a carbon star candidate by Tsalmantza et al. (2006) by its 2MASS near-IR colours. Shortly thereafter, Sloan et al. (2006) found that its IRS spectrum showed a moderately thick carbon-rich dust shell. OGLE data show that it is a Mira with a period of $\sim 670 \mathrm{~d}$ (Groenewegen 2004; Soszyński et al. 2011). It is also a known infrared variable (Polsdofer et al. 2015). We classify it here as a C-AGB, consistent with the previous works.

2MASS J00515018-7250496 (SMC IRS 103), also known as MSX SMC 125, was identified as a carbon star by Raimondo et al. (2005) based on its near-IR photometry. MACHO and OGLE both find a primary period of $\sim 460 \mathrm{~d}$ and Soszyński et al. (2011) call it a carbon-rich Mira. This source is also known to vary in the infrared (Polsdofer et al. 2015; Riebel et al. 2015). It was not in the Sloan et al. (2006) sample of SMC carbon star observations with the IRS due to contamination by Massey SMC 21202 in the IRS slit. The IRS spectrum of 2MASS J00515018-725049 was recovered in the present re-processing and is classified as a C-AGB, consistent with the optical and near-IR data.

2MASS J00542228-7243296 (SMC IRS 104), also known as MSX SMC 159, is classified as a carbon-rich AGB star by Sloan et al. (2006), based on its IRS spectrum, its period (560 d) and its luminosity $(7470 \mathrm{~L} \odot)$. It is also known to vary in the infrared (Riebel et al. 2015). Kamath et al. (2014) list this object as a candidate PN, but that is inconsistent with its firm C-AGB classification.

2MASS J00510074-7225185 (SMC IRS 105), also known as MSX SMC 163, is classified as a carbon-rich AGB star by Sloan et al. (2006), based on its IRS spectrum, its period (660 d) and its luminosity $(13000 \mathrm{~L} \odot)$. This source was also found to be infraredvariable (Polsdofer et al. 2015; Riebel et al. 2015).

IRAS 01039-7305 (SMC IRS 106), also known as 2MASS J01053027-7249536 and MSX SMC 180, was first detected with IRAS as an infrared source towards the SMC (Schwering \& Israel 1989). It was identified as being part of an OB association by Battinelli (1991), and classified as a YSO candidate based on its near-IR spectroscopy (van Loon et al. 2008). This classification was confirmed using Spitzer spectroscopy (Oliveira et al. 2013). Here, we classify it as YSO-2.

2MASS J00571098-7230599 (SMC IRS 107), also known as MSX SMC 198, is classified as a carbon-rich AGB star by Sloan 
et al. (2006), based on its IRS spectrum, its period (500 d) and it luminosity $\left(7810 \mathrm{~L}_{\odot}\right)$. This source is also known to vary in the infrared (Polsdofer et al. 2015; Riebel et al. 2015).

2MASS J00465078-7147393 (SMC IRS 108), also known as MSX SMC 200, is classified as a carbon-rich AGB star by Sloan et al. (2006), based on its IRS spectrum and its luminosity $\left(9130 \mathrm{~L}_{\odot}\right)$.

2MASS J01060330-7222322 (SMC IRS 109), also known as MSX SMC 232, is classified as a carbon-rich AGB star by Sloan et al. (2006), based on its IRS spectrum, its period (460 d) and its luminosity $(7280 \mathrm{~L} \odot$ ). It is an infrared variable (Polsdofer et al. 2015; Riebel et al. 2015).

HV 1375 (SMC IRS 110) is also known as MSX SMC 024. IR spectra (van Loon et al. 2008; Groenewegen et al. 2009; this work) identify it as an M-type AGB star. Groenewegen et al. (2009) find a spectral type of M5, a period of $418 \mathrm{~d}$, an I-band amplitude of $0.11 \mathrm{mag}$, and $\dot{M}=6.3 \times 10^{-7} \mathrm{M}_{\odot} \mathrm{yr}^{-1}$. Smith et al. (1995) detect a strong Li overabundance.

HV 11262 (SMC IRS 111), which is also known as MSX SMC 067 , is a RSG with an optical spectral type of late K or early M (Massey 2002; Massey et al. 2009), and was first noted as a possible variable by Payne-Gaposchkin \& Gaposchkin (1966). This source is also known to vary in the infrared (Polsdofer et al. 2015; Riebel et al. 2015). Its IRS spectrum is dominated by photospheric emission, and the fine structure lines longward of $15 \mu \mathrm{m}$ are probably contamination from a nearby source or the extended emission seen in the neighbourhood.

IRAS 00469-7341 (SMC IRS 112), also known as MSX SMC 079, was identified by Bolatto et al. (2007) as a candidate YSO based on its IRAC and MIPS photometry. Van Loon et al. (2008) detected $\mathrm{H}_{2} \mathrm{O}$ absorption as well as $\mathrm{Pf} \gamma$ and $\mathrm{Br} \alpha$ emission in a $3-4-\mu \mathrm{m}$ spectrum, and noted it is located near the young cluster Bruck 48. Oliveira et al. (2013) analysed its IRS spectrum as part of an IRS study of SMC YSOs and classified it with a group of early, embedded YSOs with strong ice and silicate absorption, weak PAHs, and no fine-structure lines. It is known to be an infrared variable (Polsdofer et al. 2015).

$R A W 631$ (SMC IRS 113), also known as MSX SMC 134, was classified as a carbon star from its optical spectrum by Rebeirot, Azzopardi \& Westerlund (1993). It also shows absorption features from $\mathrm{C}_{2} \mathrm{H}_{2}$ and $\mathrm{HCN}$ in the 3-4- $\mu \mathrm{m}$ region (van Loon et al. 2008), although its spectrum is somewhat atypical of the other carbon stars in that sample. Kamath et al. (2014) also list it as a carbon star. In contrast, the IRS spectrum shows strong features from crystalline silicates at $\sim 19,23,27$, and $33 \mu \mathrm{m}$, which are more typical of oxygen-rich objects. Jones et al. (2012) analyse this dual chemistry source in their sample of O-rich evolved stars, and suggest the strong crystalline silicate features are indicative of dust processing in a circumstellar disc, possibly around a binary system (Barnbaum et al. 1991; Lloyd Evans 1991). In this work, we classify it as $\mathrm{O}-\mathrm{AGB}$, because of the dominance of oxygen-rich features in the Spitzer spectrum.

2MASS J00571814-7242352 (SMC IRS 114), also known as MSX SMC 155, was classified as a R CrB candidate by Tisserand et al. (2004), based on its EROS 2 optical light curve. From Spitzer spectroscopy, Kraemer et al. (2005) confirmed that it is carbon-rich, with an almost featureless mid-infrared spectrum, characteristic of $\mathrm{R} \mathrm{CrB}$ stars. It is known to vary in the infrared (Polsdofer et al. 2015).

HV 11417 (SMC IRS 115) was classified as an SMC M supergiant by Elias et al. (1980), based on photometric and spectroscopic observations. It was the first extragalactic RSG ever identified. This source is also an infrared variable (Polsdofer et al. 2015; Riebel et al. 2015).

HV 12122 (SMC IRS 116) is an M-type AGB star (Spectral Type: M5) with a period of $544 \mathrm{~d}$ and an I-band amplitude of $1.43 \mathrm{mag}$ (Groenewegen et al. 2009). They measure a low value for $\dot{M}$ of $2.7 \times 10^{-8} \mathrm{M}_{\odot} \mathrm{yr}^{-1}$. The source is also known to vary in the infrared (Polsdofer et al. 2015; Riebel et al. 2015). Smith et al. (1995) detect a Li overabundance in the optical spectrum, indicating HBB. They also detect $\mathrm{TiO} \& \mathrm{ZrO}$, in the optical spectrum.

PMMR 34 (SMC IRS 117), which is also known as MSX SMC 096, is a RSG (e.g. Prevot et al. 1983; Elias, Frogel \& Humphreys 1985; Massey 2002). Yang \& Jiang (2012) found it to be a semiregular pulsator with a period of 381-388 d using data from the ASAS project (Pojmanski 2002). Its IRS spectrum shows weak silicate emission as well as PAH features. There is no apparent extended emission in the region, so the PAHs must be local to the source.

SkKM 71 (SMC IRS 118), also known as MSX SMC 109, is one of the many RSGs discovered by Sanduleak in the Magellanic Clouds (Humphreys 1979; Elias et al. 1985; Sanduleak 1989). Using ASAS data (Pojmanski 2002), Yang \& Jiang (2012) found it to be a 'long secondary period' variable with a period of $\sim 565 \mathrm{~d}$. Its IRS spectrum shows 9- and 18- $\mu \mathrm{m}$ silicate emission features as well as a PAH feature at $11.3 \mu \mathrm{m}$. As with PMMR 34, there is no obvious extended emission to contaminate the IRS slit so we conclude that the PAHs are local to the RSG.

HV 2084 (SMC IRS 119) is a known RSG (e.g. Prevot et al. 1983; Elias et al. 1985; Massey 2002).

HV 1652 (SMC IRS 120) was observed during an objective-prism survey of the SMC by Prevot et al. (1983). It was identified as a M0-1 supergiant.

2MASS J00463159-7328464 (SMC IRS 121), also known as MSX SMC 018, is shown to be a Mira with $P=897 \mathrm{~d}$ and $\Delta I=2.47$ mag based on its OGLE-III light curve (Soszyński et al. 2011). It is also an infrared variable (Polsdofer et al. 2015). Groenewegen et al. (2009) classify it as M-type (SpT = M7) and measure $\dot{M}=8.1 \times 10^{-6} \mathrm{M}_{\odot} \mathrm{yr}^{-1}$. van Loon et al. (2008) also classify the star as M-type via 3-4- $\mu \mathrm{m}$ spectra, consistent with our classification as $\mathrm{O}-\mathrm{AGB}$.

2MASS J00470552-7321330 (SMC IRS 122) is also known as MSX SMC 033. Spectra from Groenewegen et al. (2009), van Loon et al. (2008), and Sloan et al. (2006) all demonstrate the carbonrich AGB nature of this object with the presence of $\mathrm{C}_{2} \mathrm{H}_{2}$ features. Sloan et al. (2006) also note a SiC dust feature in the IRS spectrum. OGLE-III (Soszyński et al. 2011) measure a period of 535 d, and an $I$-band amplitude of 1.93 mag. Groenewegen et al. (2009) find that $\dot{M}=3.7 \times 10^{-6} \mathrm{M}_{\odot} \mathrm{yr}^{-1}$. It is also variable in the infrared (Riebel et al. 2015). We classify this source as C-AGB, consistent with previous studies.

2MASS J00433957-7314576 (SMC IRS 123), also known as MSX SMC 044, is a C-rich AGB star. Spectra from Groenewegen et al. (2009), van Loon et al. (2008), and Sloan et al. (2006) all confirm the presence of $\mathrm{C}_{2} \mathrm{H}_{2}$. Sloan et al. (2006) also note an $\mathrm{SiC}$ dust feature in the IRS spectrum. OGLE-III (Soszyński et al. 2011) measure a period of $440 \mathrm{~d}$, and an I-band amplitude of 1.39 mag. This source is also known to vary in the infrared (Polsdofer et al. 2015; Riebel et al. 2015). Groenewegen et al. (2009) find $\dot{M}=$ $3.1 \times 10^{-6} \mathrm{M}_{\odot} \mathrm{yr}^{-1}$. We classify this source as C-AGB, consistent with previous studies.

2MASS J00424090-7257057 (SMC IRS 124), also known as MSX SMC 062, is a C-rich AGB star. Spectra from Groenewegen et al. (2009) and Sloan et al. (2006) confirm the presence of $\mathrm{C}_{2} \mathrm{H}_{2}$. Sloan et al. (2006) also notes an $\mathrm{SiC}$ dust feature in the IRS 
spectrum. OGLE-III (Soszyński et al. 2011) measure a period of $550 \mathrm{~d}$, and an $I$-band amplitude of $1.78 \mathrm{mag}$ (Mira). Groenewegen et al. (2009) measure that $\dot{M}=1.8 \times 10^{-6} \mathrm{M}_{\odot} \mathrm{yr}^{-1}$. We classify this source as C-AGB, consistent with these earlier studies.

2MASS J00365671-7225175 (SMC IRS 125), also known as MSX SMC 091, is an AGB star with a thick carbon-rich dust shell (Sloan et al. 2006). They noted it has a particularly strong SiC emission feature for an SMC source but no $26-30-\mu \mathrm{m}$ feature. It has a very limited presence in optical catalogues, with no detections blueward of $R$ band, where it has a magnitude of $\sim 18.2$ or 18.5 (Monet et al. 2003; Lasker et al. 2008, respectively).

2MASS J00514047-7257289 (SMC IRS 126), also known as MSX SMC 142, was identified as a carbon star candidate by Raimondo et al. (2005) from its near-IR photometry. The OGLE light curve shows it is a Mira (Soszyński et al. 2011) with a period of $\sim 295$ d (Groenewegen 2004; Soszyński et al. 2011). It is also variable in the infrared (Polsdofer et al. 2015, Riebel et al. 2015). It is the weakest source in the Sloan et al. (2006) sample but clearly has a carbon-rich dust spectrum in the IRS.

2MASS J00524017-7247276 (SMC IRS 127), also known as MSX SMC 162, is classified as a carbon-rich AGB star by Sloan et al. (2006), based on its IRS spectrum, its period (520 d) and its luminosity $(11480 \mathrm{~L} \odot)$. It is also known to vary in the infrared (Polsdofer et al. 2015; Riebel et al. 2015).

2MASS J00531013-7211547 (SMC IRS 128), also known as MSX SMC 202, is classified as a carbon-rich AGB star by Sloan et al. (2006), based on its IRS spectrum and its luminosity $\left(11460 \mathrm{~L}_{\odot}\right.$ ). This source is also an infrared variable (Polsdofer et al. 2015).

2MASS J00561639-7216413 (SMC IRS 129), also known as MSX SMC 209, is classified as a carbon-rich AGB star by Sloan et al. (2006), based on its IRS spectrum, its period (520 d) and its luminosity $\left(16330 \mathrm{~L}_{\odot}\right)$. It is also in the Polsdofer et al. (2015) and Riebel et al. (2015) lists of infrared variables.

2MASS J01080114-7253173 (SMC IRS 131), also NGC 419 LE 16 , is a semiregular variable located near the cluster NGC 419. It was discovered by Lloyd Evans (1978) and classified as a carbon star based on colours. The classification is confirmed from Spitzer spectroscopy (Lagadec et al. 2007). The cluster is approximately 2 Gyr old (Kamath et al. 2010). The star shows several periods including a long secondary one; the likely fundamental period is $416 \mathrm{~d}$ (Soszyński et al. 2011). It is also variable in the infrared (Polsdofer et al. 2015).

2MASS J01082495-7252569 (SMC IRS 134), also RAW 1553 and NGC 417 LE 18, is a well-studied carbon star in the cluster NGC 419. It was first discovered by Lloyd Evans (1978) and spectroscopically observed by Rebeirot et al. (1993). The semiregular variable (Soszyński et al. 2011) has a dominant period of $181 \mathrm{~d}$ (Kamath et al. 2010).

2MASS J01082067-7252519 (SMC IRS 135), also NGC 419 LE 27, was discovered by Lloyd Evans (1978). The semiregular variable has a dominant period of $311 \mathrm{~d}$ (Soszyński et al. 2011). It shows the characteristic carbon star bands in the Spitzer SL spectrum (Lagadec et al. 2007)

2MASS J00353726-7309561 (SMC IRS 136) was discovered as a variable by Glen Moore (see Lagadec et al. 2007) using UK schmidt plates. It is classified as a carbon star on the basis of both colours (Raimondo et al. 2005) and Spitzer spectra (Lagadec et al. 2007). A $\mathrm{C} / \mathrm{O}$ ratio close to unity is indicated by the peculiar $11-\mu \mathrm{m}$ feature, lack of $\mathrm{C}_{2} \mathrm{H}_{2}$ features (Lagadec et al. 2007), and high-resolution spectra of Abia et al. (2011).
PMMR 52 (SMC IRS 137), is a well-studied SMC RSG. Massey $\&$ Olsen (2003) find $M_{\text {bol }}=-8.8 \mathrm{mag}, v_{\mathrm{rad}}=159.1 \mathrm{~km} \mathrm{~s}^{-1}$, and a spectral type of K5-7 I. The star shows low-amplitude variability with a period of $483 \mathrm{~d}$ (Yang \& Jiang 2012) and has silicate dust (Lagadec et al. 2007). It is also an infrared variable (Polsdofer et al. 2015). Note that the star should not be confused with the nebrkarby carbon Mira J005304.7-730409 (van Loon et al. 2008) which was the original but missed target of the Spitzer spectroscopy.

2MASS J00545410-7303181 (SMC IRS 139) is a 541-d Mira variable (Soszyński et al. 2011), classified as a carbon star on the basis of optical and Spitzer spectroscopy (Rebeirot et al. 1993; Lagadec et al. 2007). The 13.7- $\mu \mathrm{m}_{2} \mathrm{H}_{2}$ absorption features are absent. It is also in the Polsdofer et al. (2015) and Riebel et al. (2015) lists of infrared variables.

2MASS J00545075-7306073 (SMC IRS 140) is classified as a Mira with a period of $430 \mathrm{~d}$ (Soszyński et al. 2011), and it is also an infrared variable (Polsdofer et al. 2015; Riebel et al. 2015). It is classified as a carbon star based on photometry (Raimondo et al. 2005) and Spitzer spectroscopy (Lagadec et al. 2007).

2MASS J01015458-7258223 (SMC IRS 142) is identified as a carbon star based on the absorption bands in the Spitzer spectrum (Lagadec et al. 2007). Soszyński et al. (2011) report a period of $339 \mathrm{~d}$. The source is also known to vary in the infrared (Polsdofer et al. 2015; Riebel et al. 2015).

LEGC 105 (SMC IRS 143) is located near a small cluster, Bruck 80. The age of the cluster is $5 \times 10^{8} \mathrm{yr}$ (Chiosi et al. 2006). The star was discovered as a 310-d variable by Lloyd Evans, Glass \& Catchpole (1988, \#105 in their paper). Polsdofer et al. (2015) and Riebel et al. (2015) also find it to be an infrared variable. The Spitzer spectrum classifies it as a carbon star but there is little or no dust excess (Lagadec et al. 2007).

2MASS J00572054-7312460 (SMC IRS 144) is a 350-d Mira variable (Soszyński et al. 2011). It is also known to vary in the infrared (Polsdofer et al. 2015; Riebel et al. 2015). Lagadec et al. (2007) find the typical carbon-star bands in the Spitzer spectrum whilst Raimondo et al. (2005) classify it as a carbon star based on infrared colours.

2MASS J00555464-7311362 (SMC IRS 145), also RAW 960, is a 315-d Mira variable (Soszyński et al. 2011), classified as a carbon star based on optical photometry and spectroscopy (Rebeirot et al. 1993), using the $516 \mathrm{~nm} \mathrm{C}_{2}$ band. Lagadec et al. (2007) find little evidence for cool dust but strong molecular absorption bands of $\mathrm{C}_{2} \mathrm{H}_{2}$. This source is also an infrared variable (Polsdofer et al. 2015; Riebel et al. 2015).

IRAS 00554-7351 (SMC IRS 146) was identified by Whitelock et al. (1989) as a very long-period variable near the tip of the AGB. Soszyński et al. (2011) report a period of 610 d. The Spitzer spectra (Lagadec et al. 2007; this work) show the typical carbon-star features.

NGC 419 IR 2 (SMC IRS 147) is located in a crowded region of NGC 419. Tanabé et al. (1997) first discovered the self-obscured infrared source and the high mass-loss rate was confirmed by van Loon, Marshall \& Zijlstra (2005). The period is $381 \mathrm{~d}$ (Kamath et al. 2010). It is also in the Polsdofer et al. (2015) of infrared variables. We classify it as C-AGB.

NGC 419 IR 1 (SMC IRS 148), also known as 2MASS J01081296-7252439, is a large-amplitude (Kamath et al. 2010), 461-d Mira variable (Soszyński et al. 2011) first reported by Tanabé et al. (1997). It is classified as carbon-rich based on the Spitzer spectra (Lagadec et al. 2007; this work), and is also known to vary in the infrared (Polsdofer et al. 2015). 
$\mathrm{AzV} 404$ (SMC IRS 149) is a B2.5 yellow supergiant (Lennon 1997). It has been modelled to have a luminosity of $174000 \mathrm{~L}_{\odot}$ and light reddening of $E(B-V)=0.05 \mathrm{mag}$ (Dufton et al. 2000). We have classified it as STAR, due to the absence of emission features in the IR.

LHA 115-N38 (SMC IRS 153), also known as SMP SMC 13, is a round PN with a radius of 0.19 arcsec (Stanghellini et al. 2003a). The dust features point to a carbon-rich chemistry, and the object shows intermediate excitation lines. The temperature of the dust continuum is derived to be $T_{\text {cont }}=190 \mathrm{~K}$ (Stanghellini et al. 2007). The abundance of carbon is slightly elevated (Stanghellini et al. 2009), and LHA 115-N 38 is known to contain fullerenes (GarcíaHernández et al. 2011).

LHA 115-N 40 (SMC IRS 154), also known as SMP SMC 14, is a round PN with a radius of 0.42 arcsec, showing some internal structure (Stanghellini et al. 2003a). The Spitzer spectrum reveals carbon-rich dust features and high excitation lines, and a dust temperature of $T_{\text {cont }}=150 \mathrm{~K}$ can be derived (Stanghellini et al. 2007).

LHA 115-N 43 (SMC IRS 155), which is also known as SMP SMC 15 , is a round PN with a radius of 0.17 arcsec (Stanghellini et al. 2003a). The Spitzer spectrum shows carbon-rich dust features, intermediate excitation lines, a dust continuum with $T_{\text {cont }}=190 \mathrm{~K}$, and PAH-related emission features at $15-21 \mu \mathrm{m}$. The SiC feature is unusually broad, and the spectrum is similar to that of SMP SMC 18 and 20 (Stanghellini et al. 2007). LHA 115-N 43 is known to contain fullerenes (García-Hernández et al. 2011).

LHA 115-N 42 (SMC IRS 156), also known as SMP SMC 16 , is an elliptical PN with a radius of $0.18 \operatorname{arcsec}$ (Stanghellini et al. 2003a). The IRS spectrum shows carbon-rich dust, and lowexcitation lines, while a dust temperature of $T_{\text {cont }}=180 \mathrm{~K}$ can be derived (Stanghellini et al. 2007). The object is known to contain fullerenes (García-Hernández et al. 2011). All of this is consistent with our classification as C-PN.

LHA 115-N 44 (SMC IRS 157) is also known as SMP SMC 17. It is an elliptical PN with a radius of 0.25 arcsec, with a faint detached halo (Stanghellini et al. 2003a). The carbon-rich nature is revealed by its IRS spectrum, due to the presence of carbonrich dust. Intermediate excitation lines are present and the dust temperature is $T_{\text {cont }}=160 \mathrm{~K}$ (Stanghellini et al. 2007).

LHA 115-N 47 (SMC IRS 158), also known as SMP SMC 18, is an unresolved PN (Stanghellini et al. 2003a). It is carbon-rich in nature, and shows intermediate excitation lines. The dust temperature is derived to be $T_{\text {cont }}=170 \mathrm{~K}$ (Stanghellini et al. 2007). The $\mathrm{N}$ and O abundances are slightly low (Shaw et al. 2010), and it contains fullerenes (García-Hernández et al. 2011).

Lin 239 (SMC IRS 159), also known as Jacoby SMC 20 and SMP SMC 19 is a round PN with a radius of 0.30 arcsec, showing some outer structure (Stanghellini et al. 2003a). It is carbon-rich in nature and shows very high excitation lines. The dust temperature is measured to be $T_{\text {cont }}=150 \mathrm{~K}$ (Stanghellini et al. 2007).

LHA 115-N 54 (SMC IRS 160), also known as SMP SMC 20, is an unresolved PN (Stanghellini et al. 2003a), with a carbon-rich nebula showing intermediate excitation lines (Stanghellini et al. 2007). These last authors have also derived the dust temperature to be $T_{\text {cont }}=250 \mathrm{~K}$, which is very high, and suggest that it is one of the least evolved PNe of their sample. LHA 115-N 54 shows somewhat low N, O, Ne, S, and Ar abundances (Shaw et al. 2010), and may also contain fullerenes (García-Hernández et al. 2011), although Sloan et al. (2014) could not confirm this claim.

Lin 343 (SMC IRS 161), also known as SMP SMC 23 or Jacoby SMC 26, is a bipolar core PN with a radius of 0.30 arcsec (Stanghellini et al. 2003a). The IR spectrum shows intermedi- ate excitation lines, and a dust continuum with a temperature of $T_{\text {cont }}=150 \mathrm{~K}$ (Stanghellini et al. 2007).

Lin 357 (SMC IRS 162), also known as SMP SMC 25, is an elliptical PN with a radius of 0.19 arcsec (Stanghellini et al. 2003a). The IRS spectrum reveals an O-rich chemistry and shows very high excitation lines, on top of a dust continuum of $T_{\text {cont }}=130 \mathrm{~K}$ (Stanghellini et al. 2007). The object shows a low carbon abundance (Stanghellini et al. 2009). The progenitor was probably a high-mass star (Villaver, Stanghellini \& Shaw 2004), causing HBB.

Lin 430 (SMC IRS 163), also known as SMP SMC 26, is a point-symmetric PN with a radius of 0.28 arcsec (Stanghellini et al. 2003a). Very high excitation lines are present in the IR, on top of a dust continuum of $T_{\text {cont }}=130 \mathrm{~K}$ (Stanghellini et al. 2007). We classify it as C-PN.

LHA 115-N 87 (SMC IRS 164), also known as SMP SMC 27, is a round PN with a radius of 0.23 arcsec, and an attached outer halo (Stanghellini et al. 2003a). The IRS spectrum shows carbonrich dust features and intermediate excitation lines, on top of a dust continuum of $T_{\text {cont }}=180 \mathrm{~K}$ (Stanghellini et al. 2007). Fullerenes may also be present in this source (García-Hernández et al. 2011), although this is not confirmed by Sloan et al. (2014).

LHA 115-N2 (SMC IRS 165), also known as SMP SMC 2 or Lin 14 , is a round PN with radius of 0.25 arcsec (Stanghellini et al. 2003a). The IRS spectrum shows carbon-rich dust and very high excitation lines on a dust continuum of $T_{\text {cont }}=160 \mathrm{~K}$ (Stanghellini et al. 2007).

LHA 115-N 5 (SMC IRS 166), or SMP SMC 5 or Line 32, is a round PN with a radius of 0.31 arcsec (Stanghellini et al. 2003a). The IRS spectrum shows carbon-rich dust and very high excitation lines, on a dust continuum of $T_{\text {cont }}=180 \mathrm{~K}$ (Stanghellini et al. 2007).

LHA 115-N 7 (SMC IRS 167), also SMP SMC 8 or Lin 43, is a round PN with a radius of 0.23 arcsec (Stanghellini et al. 2003a). In the infrared, intermediate excitation lines sit on a featureless continuum of $T_{\text {cont }}=160 \mathrm{~K}$ (Stanghellini et al. 2007).

BFM 1 (SMC IRS 170). The presence of the $\mathrm{LaO}$ band at $0.79 \mu \mathrm{m}$ indicates that BFM 1 is an S star (Blanco, Frogel \& McCarthy 1981). It is known to show $\mathrm{H} \alpha$ emission (Meyssonnier \& Azzopardi 1993), and its MACHO light curve is used to derive a period of 394-400 d (Raimondo et al. 2005; Sloan et al. 2008; van Loon et al. 2008). OGLE variability data also exist (Soszyński et al. 2011). It is also an infrared variable (Riebel et al. 2015). BFM 1 is observed twice with Spitzer IRS (2.ST, 2.NO; Sloan et al. 2008) and the spectrum shows $\mathrm{ZrO}$ and $\mathrm{LaO}$ bands, and a dust emission feature at 13-14 $\mu \mathrm{m}$, which has been attributed to SiS (Sloan et al. 2011). The fitted blackbody temperature is $1460 \pm 110$ or $1160 \pm 10 \mathrm{~K}$ (Sloan et al. 2008). We have classified this as OTHER - S Star.

HV 1963 (SMC IRS 171) is a known LPV, with a spectral type consistent with an AGB star and a period of $330 \mathrm{~d}$ (Catchpole \& Feast 1981; Wood et al. 1983), in agreement with our O-EAGB classification. It is also known to vary in the infrared (Polsdofer et al. 2015). HV 1963 appears to be enhanced in lithium and $s$-process elements, while otherwise having low metallicity (Smith \& Lambert 1989). The high lithium abundance, indicative of HBB in 4-8-M $\odot$ AGB stars, is confirmed by Smith \& Lambert (1990), Plez et al. (1993), and Smith et al. (1995). The red spectrum shows moderately strong $\mathrm{TiO}$ and weak $\mathrm{CN}$ and $\mathrm{ZrO}$ bands, pointing towards carbon dredge up, and possibly envelope burning (Brett 1991). These authors also estimate that $\mathrm{C} / \mathrm{O}$ ratio is around $0.4-0.8$. The spectral type of $\mathrm{HV}$ 1963 is established to be M4.5s..., with $T_{\text {eff }}=3350 \mathrm{~K}, \log g=-0.27$ and $[\mathrm{Fe} / \mathrm{H}]=-0.43$ (Soubiran et al. 2010). HV 1963 was observed twice with Spitzer IRS. Sloan et al. (2008) report a spectral 
classification of $1 . \mathrm{N}: \mathrm{O}(:)$ and fit a blackbody temperature of $2160 \pm 120$ or $1990 \pm 50 \mathrm{~K}$. A revised period of $249 \mathrm{~d}$ is derived from the OGLE data (Sloan et al. 2008). The dust mass-loss rate is found to be $10^{-9.8} \mathrm{M}_{\odot} \mathrm{yr}^{-1}$ (Boyer et al. 2012).

HV 11329 (SMC IRS 172) is reported to be a variable O-rich AGB star (Catchpole \& Feast 1981; Wood et al. 1983), with a period of $390 \mathrm{~d}$, in agreement with our classification of O-EAGB. Lloyd Evans (1985) revised the pulsational period to $380 \mathrm{~d}$. It is also in the Polsdofer et al. (2015) and Riebel et al. (2015) lists of infrared variables. Strong Li I absorption at 6707 and $8126 \AA$ indicates HBB (Smith \& Lambert 1990; Plez et al. 1993; Smith et al. 1995). The effective temperature of this member of NGC 292 is found to be $T_{\text {eff }}=3600 \mathrm{~K}$, with $\log g=-0.05$ and $[\mathrm{Fe} / \mathrm{H}]=-0.37$ (Soubiran et al. 2010). Sloan et al. (2008) assign a spectral classification of $1 . \mathrm{NO}$, and fit a blackbody of $1600 \pm 80 \mathrm{~K}$. The MACHO data indicate a period of $377 \mathrm{~d}$ (Sloan et al. 2008; Yang \& Jiang 2012) and OGLE variability data also exist for this source (Soszyński et al. 2011).

2MASS J00445256-7318258 (SMC IRS 175). Variability data are available for this object in the OGLE and MACHO surveys, and a period of $158 \mathrm{~d}$ has been derived from the OGLE data (Groenewegen 2004), while Raimondo et al. (2005) arrive at a period of $129 \mathrm{~d}$ using the MACHO data. We classify this object as an O-AGB star. The infrared spectrum is dominated by crystalline silicate features at $\sim 19,23,28$, and $33 \mu \mathrm{m}$ originating from the circumstellar dust shell, and we classify this object as an O-AGB star, despite the presence of the 7.5 and $13.7 \mu \mathrm{m}$ absorption bands due to $\mathrm{C}_{2} \mathrm{H}_{2}$, pointing towards a carbon-rich central star. Like RAW 631, this object shows a dual chemistry (see also Kraemer et al. in preparation).

PMMR 24 (SMC IRS 176), also known as Massey SMC 11939, is known to be an RSG (e.g. Bonanos et al. 2010; Yang \& Jiang 2012). Several (inconsistent) radial velocity measurements exist (Maurice et al. 1987; Maurice, Bouchet \& Martin 1989; Massey \& Olsen 2003), and the period of $352 \mathrm{~d}$ is determined using MACHO data (Cioni et al. 2003). Atmospheric modelling of the optical spectra yields $T_{\text {eff }}=4025 \mathrm{~K}, A_{V}=1.05, \log g=0.0$, and $R=750 \mathrm{R} \odot$. Polsdofer et al. (2015) found it to be variable in the infrared.

$B M B-B 75$ (SMC IRS 177) is a dust enshrouded O-rich AGB star of spectral type M6.0 (Blanco et al. 1980), showing longperiod variability in both the MACHO (Raimondo et al. 2005) and OGLE surveys (Groenewegen 2004; Soszyński et al. 2011). It is also variable in the infrared (Polsdofer et al. 2015; Riebel et al., 2015). SiO absorption is seen in the NIR spectroscopy (van Loon et al. 2008). This object has also been observed with Spitzer's MIPSSED, and the data show a rising continuum, indicative of cold dust (42 $\pm 2 \mathrm{~K}$ ), and no emission lines (van Loon et al. 2010).

IRAS F01066-7332 (SMC IRS 178) is a dust enshrouded oxygenrich AGB star of spectral type M8 (Groenewegen \& Blommaert 1998; van Loon et al. 2008), in agreement with our classification of O-AGB. SED fitting with a dusty circumstellar shell indicates that its luminosity is $25000 \mathrm{~L} \odot$, and the mass-loss rate is $5.0 \times 10^{-7} \mathrm{M}_{\odot} \mathrm{yr}^{-1}$ (Groenewegen et al. 2000). A search for $\mathrm{SiO}$ using NIR spectroscopy does not yield any results (van Loon et al. 2008). The source is also known as an LPV (Soszyński et al. 2011), and is also known to vary in the infrared (Polsdofer et al. 2015).

RMC 50 (SMC IRS 193), also known as LHA 115-S 65, SMC V2364, and IRAS 01432-7455, is a B[e] supergiant, classified between B2 and B9, with $T_{\text {eff }}=17000 \mathrm{~K}$ and $L=500000 \mathrm{~L}_{\odot}$ (Ardeberg \& Maurice 1977; Zickgraf et al. 1986; Zickgraf 2000; Cidale, Zorec \& Tringaniello 2001; Bonanos et al. 2010; Kraus, Borges Fernandes \& de Araújo 2010). Although the star is only slightly variable, the circumstellar environment appears very dy- namic, with 2.2- $\mu \mathrm{m} \mathrm{CO}$ emission appearing during 2011 (Oksala et al. 2012).

NGC 362 SAW V16 (SMC IRS 195) is not located in the SMC, but rather a foreground object. It is an LPV (V16 designation from Hogg; see Lloyd Evans 1983a) in the Galactic globular cluster NGC $362([\mathrm{Fe} / \mathrm{H}]=-1.33$; Shetrone \& Keane 2000), hence a low-mass AGB star or a tip-RGB star. Lloyd Evans (1983a) detected significant variations in the strength of the TiO bands during the pulsation cycle equivalent to spectral type variations K4-M4. $\mathrm{He}$ also detected hydrogen emission, presumably due to pulsation shocks; McDonald \& van Loon (2007) showed the $\mathrm{H} \alpha$ to be in selfabsorption in an Échelle spectrum, at a spectral type K5.5. Lloyd Evans (1983b) determined a period of $135 \mathrm{~d}$ (138 d in Sloan et al. 2010) and an amplitude $\Delta V=2.3 \mathrm{mag}$ (similar to that of Mira variables), and confirmed cluster membership on the basis of radial velocity measurements (cf. McDonald \& van Loon 2007). It is also known to be an infrared variable (Polsdofer et al. 2015). We note that it should be an O-EAGB, but rather classify it is as OTHER, since it is a foreground object.

HV 206 (SMC IRS 196) is a foreground star, rather than an SMC object. It is an LPV (Sawyer 1931) in the Galactic globular cluster NGC $362([\mathrm{Fe} / \mathrm{H}]=-1.33$; Shetrone \& Keane 2000), hence a low-mass AGB star or a tip-RGB star (cf. Frogel, Persson \& Cohen 1983). Lloyd Evans (1983a) detected modest variations in the strength of the $\mathrm{TiO}$ bands during the pulsation cycle. He also detected hydrogen emission, presumably due to pulsation shocks (cf. Smith, Shetrone \& Keane 1999). The pulsations are semiregular with periods quoted of $90 \mathrm{~d}$ (Lloyd Evans 1983b), $89 \mathrm{~d}$ (Sloan et al. 2010), and 105 d (Lebzelter \& Wood 2011). It has been found to be lithium-rich by Smith et al. (1999). Although it is an O-EAGB, we classify this source as OTHER, since it is also a foreground object.

Massey SMC 5822 (SMC IRS 197), is known as B4 in the sample of Sheets et al. (2013) and Adams et al. (2013), who are studying OB-type stars with an 24 or $70 \mu \mathrm{m}$ excess detected by MIPS indicative of dust emission. This star is also known as source \#5822 by Massey (2002), and is assigned a group identification number of 29 in the OB sample table (table 4) of Oey, King \& Parker (2004). It has spectral type O9 (Sheets et al. 2013), and Adams et al. (2013) determine $M_{\mathrm{V}}=-4.10$ mag. We classify it as OTHER: dusty OB star.

Massey SMC 7776 (SMC IRS 198), corresponds to B9 in the sample of infrared excess emission stars (Adams et al. 2013; Sheets et al. 2013). Adams et al. (2013) determine $M_{\mathrm{V}}=-3.87 \mathrm{mag}$ and Evans et al. (2004a) assign a spectral type of B0 V. We classify it as OTHER: dusty OB star.

2MASS J00465728-7318087 (SMC IRS 199) is entry B11 in the sample of Sheets et al. (2013) and Adams et al. (2013) of MIPS-24 or MIPS-70 excess emission stars. Adams et al. (2013) determine $M_{\mathrm{V}}=-2.95$ mag. We classify it as OTHER: dusty OB star.

2MASS J00471901-7307110 (SMC IRS 200) is B14 in the sample of infrared excess emission stars (Adams et al. 2013; Sheets et al. 2013). Adams et al. (2013) determine $M_{\mathrm{V}}=-4.52 \mathrm{mag}$, and the spectral type is thought to be B0-B2 (Sheets et al. 2013). We classify it as OTHER: dusty OB star.

Massey SMC 9114 (SMC IRS 201) is also known as source B21 in the infrared excess sample of Sheets et al. (2013) and Adams et al. (2013), as source 2dFS5016 (Bonanos et al. 2010), as OGLE 04.130984 (Evans et al. 2004a), and as source 01507 by Parker et al. (1998). Adams et al. (2013) determine $M_{\mathrm{V}}=-5.00 \mathrm{mag}$, Evans $\&$ Howarth (2008) report a radial velocity of $150 \pm 6 \mathrm{~km} \mathrm{~s}^{-1}$ and Evans et al. (2004a) assign a spectral type of B1-2 II. We classify it as OTHER: dusty OB star. 
Massey SMC 9265 (SMC IRS 202), also known as OGLE SMCSC4 175188, is source B24 in the sample of OB stars with infrared excess (Adams et al. 2013; Sheets et al. 2013). Adams et al. (2013) determine $M_{\mathrm{V}}=-3.56$ mag and Evans et al. (2004a) assign a spectral type of B1-5 III. Wyrzykowski et al. (2004) report this object is part of an eclipsing binary system with a period of $P=1.3243 \mathrm{~d}$. We classify this object as OTHER: dusty OB star.

SSTISAGEMA J004752.26-732121.8 (SMC IRS 203) is entry B26 in the list of OB stars with an infrared excess (Adams et al. 2013; Sheets et al. 2013). Adams et al. (2013) determine $M_{\mathrm{V}}=-3.93 \mathrm{mag}$, and the spectral type is thought to be B0-B2 (Sheets et al. 2013). We classify this source as OTHER: dusty OB star.

Massey SMC 10129 (SMC IRS 204) is entry B29 in the sample of OB stars with an infrared excess (Adams et al. 2013; Sheets et al. 2013), and is assigned a group identification number of 50 in the OB sample table (table 4) of Oey et al. (2004). Adams et al. (2013) determine $M_{\mathrm{V}}=-5.04 \mathrm{mag}$, and the spectral type is thought to be B0 (Sheets et al. 2013). We classify this source as OTHER: dusty OB star.

2MASS J00483000-7318096 (SMC IRS 205) is entry B34 in the sample of OB stars with an infrared excess (Adams et al. 2013; Sheets et al. 2013). The spectral type is thought to be B0 (Sheets et al. 2013). We classify this source as OTHER: dusty OB star.

Massey SMC 22613 (SMC IRS 206) corresponds to B83 in the sample of OB stars with an infrared excess (Adams et al. 2013; Sheets et al. 2013). Adams et al. (2013) did not analyse this source since it is spatially resolved by Spitzer-IRS. Evans et al. (2004a) assign a spectral type of B1-5 III, while Evans \& Howarth (2008) report a radial velocity of $165 \pm 6 \mathrm{~km} \mathrm{~s}^{-1}$. We classify this source as OTHER: dusty OB star.

Massey SMC 28845 (SMC IRS 207), is entry B96 in the sample of OB stars with an infrared excess (Adams et al. 2013; Sheets et al. 2013). The spectral type is thought to be B0, and the star is known to be part of an eclipsing binary system (Sheets et al. 2013). We classify this source as OTHER: dusty OB star.

NGC 330 ELS 57 (SMC IRS 208), also known as object B100 in the sample of Sheets et al. (2013) and Adams et al. (2013), SMC5_009833 (Bonanos et al. 2010), and Massey SMC 31632, although the Spitzer spectrum is extracted $\sim 1$ arcsec away from the position of NGC 330 ELS 57. According to Evans et al. (2006), ELS 57, located 7.70 arcmin away from the centre of NGC 330, is of spectral type $\mathrm{B} 0.5 \mathrm{~V}$ and has a radial velocity of $124 \pm 4 \mathrm{~km}$ $\mathrm{s}^{-1}$. Hunter et al. (2008) assign the following stellar parameters to this star: $T_{\text {eff }}=29000 \mathrm{~K}, \log g=4.15, L=10^{4.34} \mathrm{~L}_{\odot}$, projected rotational velocity $v \sin i=104 \mathrm{~km} \mathrm{~s}^{-1}$, and $M=13 \mathrm{M}_{\odot}$. Hunter et al. (2009) determine an atmospheric microturbulent velocity for the star of $5 \mathrm{~km} \mathrm{~s}^{-1}$, and abundances of $8.01 \pm 0.18$ dex for oxygen, $6.96 \pm 0.26$ for magnesium, and $<7.48 \pm 0.29$ for nitrogen. In the study by Köhler et al. (2012), this star is known as S27, and they assign a main-sequence lifetime of $13.3 \mathrm{Myr}$, an inclination angle of $\sin i_{\mathrm{N}}=0.33$, and a 10.2 Myr isochrone age. Adams et al. (2013) determine an absolute $V$ magnitude, $M_{\mathrm{V}}$, of $-3.36 \mathrm{mag}$. We classify this object as OTHER: dusty OB star, based on these studies.

Massey SMC 32159 (SMC IRS 209), is entry B102 in the sample of dusty OB stars from Sheets et al. (2013) and Adams et al. (2013), and it is assigned a group identification number of 195 in the OB sample table (table 4) of Oey et al. (2004). Adams et al. (2013) determine $M_{\mathrm{V}}=-4.43 \mathrm{mag}$, while Sheets et al. (2013) assessing a spectral type of 09 .

2MASS J00561161-7218244 (SMC IRS 210) is entry B112 in the sample of OB stars with a far-infrared excess (Adams et al. 2013; Sheets et al. 2013). The star is known to be part of an eclipsing binary system with a period of $4.48 \mathrm{~d}$ (Bayne et al. 2002; Faccioli et al. 2007). We classify this source as OTHER: dusty OB star.

AzV 216 (SMC IRS 211) also known as Massey SMC 44984, is entry B137 in the sample of OB stars with an infrared excess (Adams et al. 2013; Sheets et al. 2013). The spectral type is thought to be B1-3II (Evans et al. 2004a) or B1 (Sheets et al. 2013), and the star is known to be part of an eclipsing binary system with a period of $1.84 \mathrm{~d}$ (Faccioli et al. 2007). We classify this source as OTHER: dusty OB star.

Massey SMC 50031 (SMC IRS 212) is entry B148 in the sample of OB stars with an infrared excess (Adams et al. 2013; Sheets et al. 2013). The spectral type is thought to be B0 (Sheets et al. 2013). We classify this source as OTHER: dusty OB star.

Massey SMC 54281 (SMC IRS 213) is entry B154 in the sample of OB stars with an infrared excess (Adams et al. 2013; Sheets et al. 2013). The spectral type is thought to be B1 (Sheets et al. 2013). We classify this source as OTHER: dusty OB star.

Massey SMC 55094 (SMC IRS 214) is entry B159 in the sample of OB stars with an infrared excess (Adams et al. 2013; Sheets et al. 2013). The spectral type is thought to be B0 (Sheets et al. 2013). We classify this source as OTHER: dusty OB star.

Massey SMC 55634 (SMC IRS 215) is also known as B161 in the sample of Sheets et al. (2013) and Adams et al. (2013). The spectral type of this object is B0 (Sheets et al. 2013). Adams et al. (2013) did not analyse this source since it is spatially resolved by Spitzer-IRS, and there is a bright source nearby in its IRS data. We classify this source as OTHER: dusty OB star.

Massey SMC 60439 (SMC IRS 216) is entry B182 in the sample of Sheets et al. (2013) and Adams et al. (2013), and it is assigned a group identification number of 391 in the OB sample table (table 4) of Oey et al. (2004). Adams et al. (2013) determine $M_{\mathrm{V}}=-4.98 \mathrm{mag}$, while Sheets et al. (2013) determine a spectral type of B0. We classify this as OTHER: dusty OB star.

Massey SMC 67470 (SMC IRS 217) is entry B188 in the sample of Sheets et al. (2013) and Adams et al. (2013). It is assigned a group identification number of 445 in the OB sample table (table 4) of Oey et al. (2004). Adams et al. (2013) determine $M_{\mathrm{V}}=-3.97 \mathrm{mag}$, while Sheets et al. (2013) believe that this is a Be star with weak $\mathrm{H} \alpha$ absorption. We group it in the OTHER: dusty OB star category.

Massey SMC 77248 (SMC IRS 218) is entry B193 in the sample of OB stars with an infrared excess (Adams et al. 2013; Sheets et al. 2013). The spectral type is thought to be B0 (Sheets et al. 2013). We classify this source as OTHER: dusty OB star.

Massey SMC 25387 (SMC IRS 219) is entry B87 in the sample of OB stars with a MIPS far-infrared excess (Adams et al. 2013; Sheets et al. 2013). It is assigned a group identification number of 149 in the OB sample table (table 4) of Oey et al. (2004). Adams et al. (2013) determine $M_{\mathrm{V}}=-4.63 \mathrm{mag}$, while Sheets et al. (2013) its spectral type to be B0. We classify this source as OTHER: dusty OB star.

Massey SMC 55681 (SMC IRS 225) is listed as an M3 star by Elias et al. (1985) and M0-M1 in the catalogue of Massey \& Olsen (2003). The latter authors also calculate $M_{\text {bol }}=-10.53$ mag, which supports our classification of RSG. Polsdofer et al. (2015) also found it to vary in the infrared.

Massey SMC 10889 (SMC IRS 226) was confirmed to be an RSG in the SMC through high-accuracy radial velocity measurements by Massey \& Olsen (2003). A spectral type of M0 Ia was assigned by Elias et al. (1985) and it is listed as having K7 I spectral type by Massey \& Olsen (2003). This all supports our classification of RSG. 
Massey SMC 11709 (SMC IRS 227). We classify this object as an RSG, in agreement with Massey \& Olsen (2003).

Massey SMC 46662 (SMC IRS 228) was identified as a late-type RSG by Levesque et al. (2007) due to the significant differences in the spectral types (M2 I to K2-3 I) and effective temperatures over short time-scales. Its location in the Hayashi forbidden zone of the $\mathrm{H}-\mathrm{R}$ diagram indicates the star is no longer in hydrostatic equilibrium and exhibits considerable variability in $V$ magnitudes. This supports our classification of RSG. It is also known to vary in the infrared (Polsdofer et al. 2015), and an X-SHOOTER spectrum is available (Chen et al. 2014).

Massey SMC 52334 (SMC IRS 229). The preliminary spectral type assigned by Elias et al. (1985) is M0 Iab, and this object is also included in the RSG catalogue of Massey \& Olsen (2003) who list the spectral class as $\mathrm{K} 7 \mathrm{I}$ and give $M_{\mathrm{bol}}=-8.15 \mathrm{mag}$. This is all in agreement with our classification of RSG. An X-SHOOTER spectrum of this object has been obtained (Chen et al. 2014).

HV 2232 (SMC IRS 230) is assigned a spectral type of M2 in the catalogue of supergiants by Elias et al. (1985) and Levesque et al. (2007). However, we classify this object as O-AGB, based on the bolometric luminosity. An X-SHOOTER spectrum of this object has been obtained (Chen et al. 2014).

HV 11423 (SMC IRS 231) is an unstable cool supergiant that was found by Massey et al. (2007) to have varied its spectral type between K0-1 I and M4.5-5 I on a time-scale of a few years. It was originally classified as an M0 supergiant by Humphreys (1979) and Elias et al. (1985) and listed by Massey \& Olsen (2003) as an RSG in the SMC. We also classify it as RSG.

Massey SMC 55188 (SMC IRS 232). Massey \& Olsen (2003) classify this source as an RSGs in the SMC, and it is also identified as an unstable cool RSG with a spectral type (M2 I-4.5 I) by Levesque et al. (2007). We classify this object as RSG. It is in the Polsdofer et al. (2015) list of infrared variables, and an X-SHOOTER spectrum has been obtained (Chen et al. 2014).

$A z V 456$ (SMC IRS 234), also known as Sk 143 (Sanduleak 1968 ), is a O9.5 or B0-1 yellow supergiant with $T_{\text {eff }}=23000$ $30000 \mathrm{~K}$ and $L=950000 \mathrm{~L} \odot$. Its spectral type was previously thought to be even earlier (O8 II:; Smith Neubig \& Bruhweiler $1997)$. The star is significantly reddened by interstellar dust to $E(B$ $-V) \approx 0.37 \mathrm{mag}$, of which $\sim 0.18 \mathrm{mag}$ is attributed to foreground Galactic dust, and hence has been used extensively to study the intervening interstellar medium (Lequeux et al. 1982; Prinja 1987; Thompson et al. 1988). The object shows a wind with a terminal velocity of $1450 \mathrm{~km} \mathrm{~s}^{-1}$ and $\dot{M}=7 \times 10^{-7} \mathrm{M}_{\odot} \mathrm{yr}^{-1}$ (Prinja 1987; Evans et al. 2004b). Membership of the SMC is confirmed on the basis of its heliocentric radial velocity $v_{\text {hel }}=166 \pm 7 \mathrm{~km} \mathrm{~s}^{-1}$ (Evans \& Howarth 2008). Its proper motion has been measured at $\approx 2 \sigma$ significance $\left(\mu_{\alpha}=6.7 \pm 3.2 \mathrm{mas} \mathrm{yr}^{-1}, \mu_{\delta}=-5.6 \pm 3.1 \mathrm{mas}\right.$ $\mathrm{yr}^{-1}$; Zacharias et al. 2004).

NGC 346 MPG 293 (SMC IRS 235) is a B3 Ia (Azzopardi \& Vigneau 1982), B1 Ia (Bouchet et al. 1985), or B2 Ia (Smith Neubig \& Bruhweiler 1997) supergiant in the young massive cluster NGC 346. It has a relatively slow line-driven wind $\left(v_{\infty} \approx 900 \mathrm{~km}\right.$ $\mathrm{s}^{-1}$; Prinja 1987), attributed to the low metal content.

AzV 23 (SMC IRS 236) is a B3 Ia (Azzopardi \& Vigneau 1982; Lennon 1997) yellow supergiant, first catalogued as Sk 17 (Sanduleak 1968). Membership of the SMC is confirmed on the basis of its heliocentric radial velocity $v_{\text {hel }}=178 \mathrm{~km} \mathrm{~s}^{-1}$ (Neugent et al. 2010, who derived a spectral type of B2 I). It has been modelled to have a luminosity of $230000 \mathrm{~L}_{\odot}$ and moderate reddening of $E(B$ $-V)=0.21 \mathrm{mag}$ (Dufton et al. 2000).
IRAS 00350-7436 (SMC IRS 238), also known as LI-LMC 5, is a carbon-rich, high-luminosity object $\left(M_{\mathrm{bol}}=-6.564\right.$ to $-6.82 \mathrm{mag}$; Whitelock et al. 1989; van Loon et al. 1998; Tsalmantza et al. 2006). Zijlstra et al. (1996) designate it as a candidate AGB star, with a dust mass-loss rate derived from IR colours of $10^{-8.17}$ $10^{-7.25} \mathrm{M}_{\odot} \mathrm{yr}^{-1}$, depending on the specific colour. It is among the most luminous AGB stars in the Magellanic Clouds, with only one object in the LMC having similar luminosity, IRAS 04496-6958 (van Loon et al. 1998). Matsuura et al. (2005) suggest that this is a post-AGB star, based on the presence of the 3.3- $\mu \mathrm{m}$ PAH emission feature. Because of the presence of weak $\mathrm{C}_{2} \mathrm{H}_{2}$ absorption in the IRS spectrum, we classify it as C-AGB.

NGC 330 SW 515 (SMC IRS 239) is an irregular optical variable (Sebo \& Wood 1994), with a possible 45-d orbital period but also variability on both shorter and longer time-scales (Schmidtke, Chobanian \& Cowley 2008), and a possible member of the cluster NGC 330. It features broad $\mathrm{H} \alpha$ emission (Meyssonnier \& Azzopardi 1993; Oliveira et al. 2013), which is double peaked (Hummel et al. 1999), as well as near-IR hydrogen emission (Tanabé et al. 2013). Martayan et al. (2007) classified it as a HeB[e] object. Mid-IR excess emission was detected with the ISOby Kučinskas et al. (2000) and with Spitzer by Bolatto et al. (2007). The Spitzer IRS spectrum was first published by Oliveira et al. (2013), who recognized it as a young star but with no trace of $\mathrm{H}_{2} \mathrm{O}$ or $\mathrm{CO}_{2}$ ices.

Lin 517 (SMC IRS 240) is also known as [BSS2007 282] and LHA 115-N 86. It appears in earlier lists of $\mathrm{H} \alpha$ sources and possible $\mathrm{PNe}$, e.g. Henize (1956) and Lindsay (1961). Kamath et al. (2014) find that it may be a hot post-AGB star. We classify this object as YSO-4.

[MA93] 1771 (SMC IRS 241) is also 2MASS J01134116-7250499 and SSTISAGEMA J011341.20-725049.8. It is a known $\mathrm{H} \alpha$ source (Meyssonnier \& Azzopardi 1993), who believe it to be the red component of a 2 arcsec pair, and possibly related to Lin 497. Indeed, the MCPS survey (Zaritsky et al. 2002) shows three potential counterparts within 3 arcsec of the SAGE-SMC position (Gordon et al. 2011). This very red source is also detected within 3 arcsec by Herschel in the Herschel Inventory of the Agents of Galaxy Evolution survey (HERITAGE; Meixner et al. 2013) at 100, 160, and $250 \mu \mathrm{m}$. Kamath et al. (2014) find that this object may be a hot post-AGB star.

2MASS J00540342-7319384 (SMC IRS 242) is object 18 in the spectral catalogue by Oliveira et al. (2013). It is identified as a YSO candidate based on MIPS SED data (van Loon et al. 2008) and the IRS spectrum (Oliveira et al. 2011; this work).

2MASS J01054645-7147053 (SMC IRS 243). Initially proposed as a YSO (Bolatto et al. 2007), this object has since been classified as a carbon-rich proto-PN by Volk et al. (2011), who point out the strong mid-IR emission features usually attributed to PAHs, and a hint of a weak 21- $\mu \mathrm{m}$ emission feature of which the carrier remains unknown. They derived a luminosity of $4660 \pm 510 \mathrm{~L}_{\odot}$ and birth mass of only $1.00_{-0.04}^{+0.12} \mathrm{M}_{\odot}$ - which seems low for the carbon enrichment. Kamath et al. (2014) derive $L=4106 \mathrm{~L} \odot$ and, based on the presence of atomic emission lines and a UV continuum, conclude that this is a hot post-AGB star.

2MASS J00540236-7321182 (SMC IRS 244) is object 17 in the spectral catalogue by Oliveira et al. (2013). It is identified as a YSO candidate based on MIPS SED data (van Loon et al. 2008) and the IRS spectrum (Oliveira et al. 2011; this work).

OGLE SMC-SC10 107856 (SMC IRS 245) is a (strong) candidate R CrB type star (Tisserand et al. 2009), experiencing erratic, sudden obscuration events by circumstellar carbonaceous dust clouds. Polsdofer et al. (2015) and Riebel et al. (2015) list it as an infrared 
variable. It has a relatively cool atmosphere, $\sim 5000 \mathrm{~K}$ compared to most R CrB stars.

IRAS 00516-7259 (SMC IRS 246) is entry 16 in the spectral catalogue of YSO candidates by Oliveira et al. (2013).

IRAS 00509-7342 (SMC IRS 247) is entry 15 in the spectral catalogue of YSO candidates by Oliveira et al. (2013), who also report extended radio continuum emission at 1.42, 2.37, 4.80, and $8.64 \mathrm{GHz}$ at the same position. Kamath et al. (2014) confirm the YSO nature of this source.

2MASS J00505814-7307567 (SMC IRS 248) is entry 14 in the catalogue by Oliveira et al. (2013). It is also known as an $\mathrm{H} \alpha$ emitter (Meyssonnier \& Azzopardi 1993). Kamath et al. (2014) confirm the YSO nature of this source.

2MASS J00504326-7246558 (SMC IRS 249) is entry 13 in the spectral catalogue of YSO candidates by Oliveira et al. (2013), while it is also known as an $\mathrm{H} \alpha$ emitter (Meyssonnier \& Azzopardi 1993). It appears to be part of a star cluster (Bica \& Schmitt 1995; Chiosi et al. 2006). Kamath et al. (2014) confirm the YSO nature of this source.

2MASS J00504042-7320369 (SMC IRS 250) is entry 12 in the spectral atlas by Oliveira et al. (2013).

2MASS J00494469-7324331 (SMC IRS 251) is entry 11 in the spectral atlas by Oliveira et al. (2013).

SMP SMC 21 (SMC IRS 252) is a well-known SMC PN first identified by (Lindsay 1961). Our classification as an O-rich PN is consistent with the abundance analyses available in the literature (i.e. Leisy \& Dennefeld 1996).

Massey SMC 60447 (SMC IRS 253) was first catalogued by Basinski, Bok \& Bok (1967) where it is object 353 in their table V. The object was then classified as an RSG star in Sanduleak (1989) where it is object 276; the positions are slightly different in these two papers but it is clearly the same object. The spectral type is given as K2I in Massey (2002). This is all consistent with our classification of RSG. The source is also known to vary in the infrared (Polsdofer et al. 2015).

PMMR 145 (SMC IRS 254) was first noted by Prevot et al. (1983), as a RSG of spectral type K5-K8. The object is also discussed by Elias et al. (1985). An abundance analysis was carried out by Hill (1997), who gives parameters $T_{\text {eff }}=4300 \mathrm{~K}, \log g=+0.3$, and $[\mathrm{M} / \mathrm{H}]=-0.6$ along with a radial velocity of $160.6 \mathrm{~km} \mathrm{~s}^{-1}$. Analysis of the CNO abundances by Hill, Barbuy \& Spite (1997) yields a $\mathrm{C} / \mathrm{O}$ ratio of 0.3 . All of these observations are consistent with our classification of the object as RSG.

PMMR 141 (SMC IRS 255). Prevot et al. (1983) catalogue this star as a late-type supergiant. The spectral type is given as K7-M0I by Massey \& Olsen (2003). All the references to this object in the literature are consistent with our classification of the object as RSG.

PMMR 132 (SMC IRS 256) was catalogued as a late-type supergiant in the SMC by Prevot et al. (1983) based upon objective prism observations. Photometry is given in Elias et al. (1985). We classify it as RSG.

LHA 115-S 38 (SMC IRS 257) was identified as an emission line object by Henize (1956). It was then also catalogued by Lindsay (1961) as Lin 418. The spectral type is given as between A3 and F0 in Evans et al. (2004a, object 1804). The star is also discussed by Raimondo et al. (2005) as a likely carbon-rich object based on the $J$ $-K$ colour. Kamath et al. (2014) identify this object as a post-AGB candidate (\# 38 in their Q1 list), showing a slow brightening in the optical combined with a long period of $\sim 900 \mathrm{~d}$, possibly due to changes in the dust obscuration or the accretion rate. The IRS spectrum, reveals its O-rich nature and we are able classify this object as O-PAGB.
$R A W 594$ (SMC IRS 258) was identified as a probable carbonrich variable star by Rebeirot et al. (1993) based on its near-infrared colours. There are four other papers in the literature concerning the variability or SED properties of the object, which is a semiregular pulsator from the OGLE survey. Our IRS spectrum classification confirms the carbon-rich nature and we classify it as C-AGB.

2MASS J00444463-7314076 (SMC IRS 259). Nothing specific is known about this object, except that it is an infrared variable (Polsdofer et al. 2015).

SSTISAGEMA J005419.21-722909.7 (SMC IRS 260) was identified based on its IRAC colours as a YSO candidate by Bolatto et al. (2007), as YSO candidate 139 in their list ([BSS2007] 139), and as such included in the YSO sample to be observed with Spitzer-IRS by Oliveira et al. (2013, entry \#19). However, these authors find that this object is not a YSO, but rather a D-type symbiotic star, consisting of an AGB star and white dwarf, with mass transfer between them. There is also a nearby blue star (about 2.5 arcsec away from the symbiotic binary) contributing a continuum to the spectrum that cannot be separated out (Oliveira et al. 2013).

2MASS J00432649-7326433 (SMC IRS 261) is a semiregular variable carbon star (Raimondo et al. 2005; Soszyński et al. 2011). It is also in the Polsdofer et al. (2015) list of infrared variables.

Lin 250 (SMC IRS 262), also known as LHA 115-S 18, is a wellknown $\mathrm{B}[\mathrm{e}]$ supergiant (see e.g. Clark et al. 2013 and references therein for a comprehensive view of this system). It is an infrared variable (Polsdofer et al. 2015; Riebel et al. 2015).

IRAS 00471-7352 (SMC IRS 263), is known to be an LPV (Groenewegen 2004; Raimondo et al. 2005; Soszyński et al. 2011), carbon-rich in nature (Raimondo et al. 2005). It is also an infrared variable (Polsdofer et al. 2015).

SSTISAGEMA J004901.61-731109.5 (SMC IRS 264) is entry 10 in the Spitzer-IRS spectral atlas of YSO candidates by Oliveira et al. (2013).

LHA 115-N 31 (SMC IRS 265), also known as Lin 120, is an emission line star (Henize 1956; Meyssonnier \& Azzopardi 1993). It is classified as a candidate YSO by Bolatto et al. (2007), while Charmandaris, Heydari-Malayeri \& Chatzopoulos (2008) included it in their sample of candidate compact $\mathrm{H}$ II regions, as object \#5, and remark that the IRAC colours set it apart from class I and class II YSOs. LHA 115-N 31 is also entry 9 in the Spitzer-IRS spectral atlas of YSO candidates by Oliveira et al. (2013).

Lin 60 (SMC IRS 266) is also known as LHA 115-N 10 (see e.g. Meyssonnier \& Azzopardi 1993). It was one of the sample with MIPS spectra obtained by van Loon et al. (2010), and Oliveira et al. (2013) examined it as a bright YSO as well. Its spectrum has clear $\mathrm{CO}_{2}$ ice absorption at $15 \mu \mathrm{m}$. It appears in older lists, such as Henize (1956) and Lindsay (1961).

S3MC J004825.83-730557.29 (SMC IRS 267) is found in a complex region, and is therefore not identified as a point source by the SAGE-SMC team (Gordon et al. 2011), but its flux levels were extracted by the S3MC team (Bolatto et al. 2007), who also classify it as a candidate YSO. This is entry \#8 in the IRS spectral catalogue of YSO candidates by Oliveira et al. (2013), who report detections of $\mathrm{CO}_{2}$ ice and PAHs. Kamath et al. (2014) offer an alternative view by classifying this as a candidate PN. We classify this object as YSO-1.

2MASS J00444111-7321361 (SMC IRS 268) is a smallamplitude semiregular variable star, with $P \approx 98 \mathrm{~d}$ (Soszyński et al. 2011), later refined to $96.338 \mathrm{~d}$ (Kamath et al. 2014). While it was selected as a candidate YSO on the basis of mid-IR photometry (Bolatto et al. 2007), it has subsequently been shown to be a postAGB object on the basis of its $21-\mu \mathrm{m}$ emission feature (Volk et al. 
2011). The post-AGB nature was confirmed by the measurement of extreme $s$-process elemental enhancements, its carbon enrichment (with $\mathrm{C} / \mathrm{O}>1$ ), and iron depletion, but not the otherwise expected lead overabundance; a birth mass of $\sim 1.3 \mathrm{M}_{\odot}$ was inferred (De Smedt et al. 2012, 2014).

2MASS J00465185-7315248 (SMC IRS 269) appears to be slightly extended at IRAC wavelengths, and is therefore not identified as a point source by the SAGE-SMC team (Gordon et al. 2011). The S3MC team (Bolatto et al. 2007) was able to fit a point source and extract the flux levels. They also classify it as a candidate YSO. This is entry \#7 in the IRS spectral catalogue of YSO candidates by Oliveira et al. (2013), who report the presence of PAH emission.

S3MC J004624.46-732207.30 (SMC IRS 270) is a YSO candidate (Bolatto et al. 2007, \#43), observed with MIPS SED (van Loon et al. 2010, \#3) and IRS (Oliveira et al. 2013, \#6).

SSTISAGEMA J004547.53-732142.1 (SMC IRS 271) is entry 5 in the IRS spectral catalogue by Oliveira et al. (2013). It is an infrared variable (Polsdofer et al. 2015).

2MASS J00452129-7312185 (SMC IRS 272) is entry 4 in the IRS spectral catalogue by Oliveira et al. (2013). It also appears in the emission-line star catalogue by Meyssonnier \& Azzopardi (1993).

IRAS 00429-7313 (SMC IRS 273), also known as LI-SMC 25, was originally thought of as an AGB star candidate, based on its IRAS detection (Loup et al. 1997). More recently, it was recognized as an early-type YSO candidate, and it is included in the spectral catalogues by van Loon et al. (2010, \#1; who also provide a short literature review on this source) and Oliveira et al. (2013, \#2). Kamath et al. (2014) also list it as YSO candidate, showing optical variability in the form of Cepheid-like small oscillations, with a period of $22.5 \mathrm{~d}$.

LHA 115-N 8 (SMC IRS 274), also known as Lin 41, is entry \#1 in the IRS spectral catalogue of YSO candidates by Oliveira et al. (2013). It is also known as an emission-line star (Meyssonnier \& Azzopardi 1993).

2MASS J01061966-7155592 (SMC IRS 275) is catalogued as a YSO candidate by Bolatto et al. (2007, entry \#257). Based on its UV continuum and emission-line characteristics, Kamath et al. (2014) also list this as a YSO candidate.

2MASS J01052863-7159426 (SMC IRS 276) is catalogued as a YSO candidate by Bolatto et al. (2007, entry \#251). Based on its UV continuum and emission-line characteristics, Kamath et al. (2014) also list this as a YSO candidate.

HV 12956 (SMC IRS 277) is a luminous ( $M_{\mathrm{bol}} \sim-6.5 \mathrm{mag}$ ), Miratype variable with a period of 518 d (Catchpole \& Feast 1981; Wood et al. 1983; Soszyński et al. 2011; Yang \& Jiang 2012). Polsdofer et al. (2015) also list it as an infrared variable. This M5e-type AGB star was identified with the mid-IR source IRAS 01074-7140 (Whitelock et al. 1989; Zijlstra et al. 1996; Groenewegen \& Blommaert 1998; van Loon et al. 1998), and the mid-IR spectrum obtained with the $I S O$ satellite was analysed by Groenewegen et al. (2000). Despite being one of the better candidates in the SMC for circumstellar maser emission, none was detected in deep searches (van Loon et al. 2001). The star was found to be lithium-rich, probably as a result of nuclear processing at the base of the convection zone (Smith et al. 1995). A 3-4 $\mu \mathrm{m}$ spectrum was presented in van Loon et al. (2008).

2MASS J01035898-7255327 (SMC IRS 278) is entry \#238 in the YSO candidate list by Bolatto et al. (2007).

LHA 115-N 61 (SMC IRS 279), also known as Lin 321; IRAS 00557-7248 and LI-SMC 121; this is a well-known emission line nebula in the SMC first discovered by Henize (1956). It was classi- fied as a PN by Lindsay (1961) based on a lack of detected optical continuum around $\mathrm{H} \alpha$ and the presence of the [N II] 6548/6584 forbidden lines. For this reason, the object has sometimes been considered as a PN in the literature (i.e. Jacoby \& De Marco 2002), although it was classified as an $\mathrm{H}$ II region by Henize \& Westerlund (1963) and this was confirmed spectroscopically by Dufour \& Killen (1977).

Lin 238 (SMC IRS 280) is a known emission-line star (Lindsay 1961; Meyssonnier \& Azzopardi 1993). More recently it was included in the catalogue by Bolatto et al. (2007, object 132) as a YSO candidate. It is an infrared variable (Polsdofer et al. 2015).

HD 5980 (SMC IRS 281) is a famous 19-d eclipsing WR binary (Breysacher \& Perrier 1980; Breysacher, Moffat \& Niemela 1982) with estimated masses of $M_{\mathrm{A}}=58-79 \mathrm{M}_{\odot}$ and $M_{\mathrm{B}}=51-67 \mathrm{M}_{\odot}$, respectively (Foellmi et al. 2008). Koenigsberger et al. (2014) report that the system has in fact a third component, which is an O-star in an eccentric orbit with a period of $96.56 \mathrm{~d}$. HD 5980 had already been recognized as peculiar well over a century ago (Pickering \& Fleming 1901), and was identified as an emission-line star half a century later (Henize 1956), with variable emission-line width (Feast, Thackeray \& Wesselink 1960). It resides in the massive cluster NGC 346, but its proper motion of ( $\mu_{\alpha}=-3.50 \pm 1.70$ mas $\mathrm{yr}^{-1}, \mu_{\delta}=-2.40 \pm 1.60$ mas $\mathrm{yr}^{-1}$; Hog et al. 1998) is marginally significant. Component $\mathrm{A}$, of WN type, underwent luminous blue variable (LBV)-type eruptions in 1993 and 1994 (Barba et al. 1995, 1996; Cellone et al. 1996; Eenens \& Morris 1996; Koenigsberger et al. 1995, 1996, 1998a,b; Heydari-Malayeri et al. 1997; Sterken \& Breysacher 1997; Moffat et al. 1998), creating a circumstellar shell (Dopita et al. 1994; Morris et al. 1996; Koenigsberger et al. 2000, 2001; González \& Koenigsberger 2014), as well as showing longer term S Doradus-type variability (Koenigsberger et al. 2010; Georgiev et al. 2011) and spectral changes preceding the outburst (Koenigsberger et al. 1994). HD 5980 is a bright, variable X-ray source (Nazé et al. 2004; Guerrero \& Chu 2008) as a result of colliding winds (Breysacher \& François 2000; Koenigsberger 2004; Koenigsberger et al. 2006; Koenigsberger \& Moreno 2008); polarimetric variability indicates complex structure and/or a neutron star companion (Villar-Sbaffi, Moffat \& St-Louis 2003). Indeed, the supernova remnant SNR 0057-7226 lies in the direction towards HD 5980 (Hoopes et al. 2001; Nazé et al. 2002; Velázquez, Koenigsberger \& Raga 2003). The binary was detected at mid-IR wavelengths with ISO by Contursi et al. (2000); the Spitzer midIR photometric properties were analysed by Bonanos et al. (2010), who point out that this is the only WR star (but not the only LBV) in the SMC with a detection at $24 \mu \mathrm{m}$. Being such bright, earlytype, line-broadened star, HD 5980 has been used extensively as a background probe for interstellar medium studies (despite its low $E(B-V)=0.05 \mathrm{mag}-$ Schmutz \& Vacca 1991) - e.g., polarization (Mathewson \& Ford 1970; Schmidt 1970); UV (de Boer \& Savage 1980; Savage \& de Boer 1981; Fitzpatrick \& Savage 1983, 1985; Sembach \& Savage 1992) including detection of $\mathrm{H}_{2}$ (Richter et al. 1998; Shull et al. 2000) - see also Cohen (1984), Songaila et al. (1986), Sembach, Danks \& Savage (1993), Lipman \& Pettini (1995), and Hoopes et al. (2002). Polsdofer et al. (2015) list it as an infrared variable.

HD 6884 (SMC IRS 282) (or R 40) is a luminous blue supergiant (Feast et al. 1960) of type B9 Iae (Garmany, Conti \& Massey 1987; B8 Ia based on UV - Smith Neubig \& Bruhweiler 1997) displaying $\mathrm{H} \alpha$ line emission (LHA 115-S 52; Henize 1956), with confirmed membership of the SMC based on a radial velocity of $170 \mathrm{~km}$ $\mathrm{s}^{-1}$ (Buscombe \& Kennedy 1962). It is the visually brightest B star (Stahl et al. 1985) and the first recognized LBV in the SMC (Szeifert 
et al. 1993), explaining the later spectral type of A2 determined by (Lennon 1997) and the S Dor-type variability (Sterken, de Groot \& van Genderen 1998). Early optical photometry was presented by Dachs (1970), Osmer (1973), Ardeberg \& Maurice (1977), and van Genderen, van Leeuwen \& Brand (1982); a $K$-band spectrum was presented by Oksala et al. (2013). Not surprisingly, it has been used extensively for studies of the interstellar medium (e.g. Cohen 1984; Songaila et al. 1986); the 4430- $\AA$ diffuse interstellar band was detected in its spectrum by Hutchings (1966), and the degree of polarization was measured by Mathewson \& Ford (1970). Bonanos et al. (2010) detected mid-IR excess emission on the basis of Spitzer photometry. It is in the Polsdofer et al. (2015) list of infrared variables.

2MASS J00531330-7312176 (SMC IRS 283) is entry \#131 in the YSO candidate list by Bolatto et al. (2007). The source is possibly also detected in the far-infrared (Wilke et al. 2003), as [WSH2003] b-63, although the coordinates are very coarse at these wavelengths. Within 10 arcsec, another far-infrared detection with Herschel has also been reported, again with rather imprecise coordinates (Meixner et al. 2013).

2MASS J00531330-7312176 (SMC IRS 284) is entry \#129 in the YSO candidate list by Bolatto et al. (2007).

2MASS J00505425-7324170 (SMC IRS 286) is YSO candidate 112 in the catalogue by Bolatto et al. (2007).

LHA 115-N 32 (SMC IRS 287) LHA 115-N 32 is a well-known source, with more than 20 references, starting with Henize (1956) and Lindsay (1961). It has been characterized more as an $\mathrm{H}$ II region than a PN (most recently by Charmandaris et al. 2008). We classify the spectrum as YSO-3, but remark that it is due from a source located within an $\mathrm{H}$ II region.

2MASS J00484901-7311226 (SMC IRS 288) is entry \#83 in the list of YSO candidates by Bolatto et al. (2007). In contrast, Kamath et al. (2014) catalogue this object as a hot post-AGB candidate. We classify it as YSO-3.

2MASS J00465576-7331584 (SMC IRS 289) is entry \#47 in the list of YSO candidates by Bolatto et al. (2007). Kamath et al. (2014) also list this object as a candidate YSO.

2MASS J00454296-7317263 (SMC IRS 290) is entry \#27 in the list of YSO candidates by Bolatto et al. (2007).

IRAS 01042-7215 (SMC IRS 291). Wilke et al. (2003) determined the 25- and 60- $\mu \mathrm{m}$ flux densities from high-resolution IRAS maps (source b-97), to be $F_{25}=0.6 \pm 0.1$ and $F_{60}=8.4 \pm 0.3 \mathrm{Jy}$ (cf. Schwering \& Israel 1989); they did not detect the source in their ISO-PHOT maps at a wavelength of $170 \mu \mathrm{m}$. A far-IR spectrum obtained with the Spitzer SED mode was presented by van Loon et al. (2010). The near-IR counterpart was found by Groenewegen \& Blommaert (1998). While Groenewegen et al. (2000) modelled the ISO mid-IR data as if it were a mass-losing cool oxygen-rich AGB star, van Loon et al. (2008) classified it as a candidate YSO on the basis of a $3-4-\mu \mathrm{m}$ spectrum displaying water ice absorption and hydrogen recombination emission. Indeed, Oliveira et al. (2011) detected (weak) $\mathrm{CO}_{2}$ ice absorption in the Spitzer IRS spectrum confirming the YSO nature of this object; $\mathrm{CO}$ ice was not detected in their ground-based $M$-band spectrum. Oliveira et al. (2013) also presented an optical spectrum showing just broad $\mathrm{H} \alpha$ emission, as well as new near-IR photometry $\left(J K_{S} L^{\prime}\right)$. Polsdofer et al. (2015) list it as an infrared variable.

Lin 49 (SMC IRS 292) has been known as an emission line source and/or a PN (Lindsay 1961; Henize \& Westerlund 1963; Dopita et al. 1985; Meyssonnier \& Azzopardi 1993; Morgan 1995). While Bolatto et al. (2007) included it in their YSO candidate list (\#7), the IRS spectrum clearly shows that this is a C-PN, and a fullerene source (Sloan et al. 2014; this work).

2MASS J00431490-7300426 (SMC IRS 294) is entry \#4 in the list of YSO candidates by Bolatto et al. (2007).

NGC 330 BAL 555 (SMC IRS 296) is another member of NGC 330 , and is known to be a long-period semiregular variable from OGLE observations (Soszyński et al. 2011). The object appears to have first been catalogued as a member of NGC 330 by Balona (1992) where it is object 555 in Table 2. Kamath et al. (2014) list it as a carbon star, consistent with our classification of this object as C-AGB.

2MASS J01065966-7250430 (SMC IRS 298) is an emission-line star (Meyssonnier \& Azzopardi 1993), listed as a candidate PN by Kamath et al. (2014). van Loon et al. (2010) list it as a YSO (entry 11 ), and the IRS spectrum confirms the YSO nature of this object (Oliveira et al. 2013, \#30). We classify it as YSO-3.

2MASS J01053088-7155209 (SMC IRS 299) is entry 29 in the IRS spectral catalogue of YSO candidates by Oliveira et al. (2013).

2MASS J01050732-7159427 (SMC IRS 300) is entry 8 in the study by van Loon et al. (2010), who list it as a YSO, and provide some further references. Its YSO nature is confirmed by its IRS spectrum (Oliveira et al. 2013, \#28).

S3MC J010306.13-720343.95 (SMC IRS 301) is entry 228 in the YSO candidate list by Bolatto et al. (2007), and its YSO nature is confirmed by its IRS spectrum (Oliveira et al. 2013, \#27).

S3MC J010248.54-715317.98 (SMC IRS 302) is a mid-infrared point source (also detected by the SAGE team as SSTISAGEMA J010248.56-715318.0) part of star forming region A8 discovered by (Livanou et al. 2007), which is about 240 pc in size. Region A8 contains several $\mathrm{H}$ II regions, and S3MC J010248.54-715317.98 is located inside one of these $\mathrm{H}$ II regions, namely DEM S 117b, also known as LHA 115-N 77A (Bica \& Schmitt 1995), which has a size of $0.55 \mathrm{arcmin}$. The IRS spectrum therefore shows many emission lines due to the $\mathrm{H}$ in region. We conclude that the point source is an YSO-3 object located within an $\mathrm{H}$ II region (see also Oliveira et al. 2013, \#26).

S3MC J010131.69-715040.30 (SMC IRS 303) is entry 25 in the IRS spectral catalogue of YSO candidates by Oliveira et al. (2013), and entry 210 in the list of YSO candidates of Bolatto et al. (2007).

SSTISAGEMA J010022.34-720957.8 (SMC IRS 304) is entry 24 in the IRS spectral catalogue of YSO candidates by Oliveira et al. (2013), and it is also entry 60 in the list of YSOs in N66 compiled by Simon et al. (2007), who derive $L=2910 \mathrm{~L}_{\odot}$ and $M=8 \mathrm{M}_{\odot}$ for this source. It is also known to vary in the infrared (Polsdofer et al. 2015; Riebel et al. 2015).

IRAS 00563-7220 (SMC IRS 305) is entry 23 in the IRS spectral catalogue of YSO candidates by Oliveira et al. (2013). It is also \#174 in the YSO candidate list published by Bolatto et al. (2007).

IRAS 005627255 (SMC IRS 306) is entry 22 in the IRS spectral catalogue of YSO candidates by Oliveira et al. (2013). It is also \#171 in the YSO candidate list published by Bolatto et al. (2007).

2MASS J00560662-7247225 (SMC IRS 307) is reported to be an emission-line star (Meyssonnier \& Azzopardi 1993), perhaps consisting of multiple components. It is also entry 21 in the IRS spectral catalogue of YSO candidates by Oliveira et al. (2013). Bolatto et al. (2007) list it as \#146 in their list of YSO candidates, and it is also included as a YSO candidate in the work by Kamath et al. (2014). We classify it as YSO-1.

HV 11464 (SMC IRS 309) is first classified as M0 I by Prevot et al. (1983) and Elias et al. (1985); SMC membership was confirmed based on the heliocentric radial velocity of $v_{\text {hel }}=189 \mathrm{~km} \mathrm{~s}^{-1}$ (Maurice et al. 1987); however, the spectral type was revised to K0 I 
and the radial velocity to $182 \mathrm{~km} \mathrm{~s}^{-1}$ by Massey \& Olsen (2003), who also determined a bolometric luminosity of $M_{\mathrm{bol}}=-8.0 \mathrm{mag}$. Yang \& Jiang (2012) determined a long secondary period of 1500 1600 d. The Spitzer mid-IR photometry was analysed by Bonanos et al. (2010). It straddles the boundary between the RSG and AGB classification, and because of its $M_{\text {bol }}$ value we classify it as O-AGB.

IRAS 00436-7321 (SMC IRS 310) is in a complex field. The source is also an emission-line star and listed as entry 108 in the $\mathrm{H} \alpha$ survey by Meyssonnier \& Azzopardi (1993). The IRAS name is cross-identified with this Spitzer source, as it is the only source with a MIPS-[24] flux level comparable to the IRAS-[25] flux within the error box of the IRAS-[25] position (no detection at IRAS-[12]). Keller et al. (in preparation) believe this source to be a PN, however we classify it as $\mathrm{H}$ II.

NGC 330 ARP 41 (SMC IRS 311), a member of the NGC 330 cluster, has been known to be a late-type supergiant since the 1970's. The star was first observed by Arp (1959) as object 41 in his list. The spectral type was determined as G6Ib by Feast (1979). Our classification of this object as a RSG is (marginally) consistent with this spectral type.

This paper has been typeset from a $\mathrm{T}_{\mathrm{E}} \mathrm{X} / \mathrm{L} \mathrm{A} \mathrm{T}_{\mathrm{E}} \mathrm{X}$ file prepared by the author. 